\title{
Particle-Transport Simulation with the monte Carlo method
}

L. L. Carter and E. D. Cashwell

Los Alamos Scientific Laboratory

This report was prepared as an account of work
sponsored by the United States Government. Neither
the United States not the United States Energy
Research and Development Administration, nor any of
their employes, nor any of their contractors,
subcontractors, or their employees, makes any
warranty, express or implied, or assumes any legal
kability or responsibility for the accuracy, completeness
or usefulness of any information, apparat us, product or
process disclosed, or represents that its use would not
infringe privately owned rights.

Prepared for the Division of Military Application U. S. Energy Research and Development Administration

Published by Technical Information Center, Office of Public Affairs U. S. Energy Research and Development Administiration 


\section{DISCLAIMER}

This report was prepared as an account of work sponsored by an agency of the United States Government. Neither the United States Government nor any agency Thereof, nor any of their employees, makes any warranty, express or implied, or assumes any legal liability or responsibility for the accuracy, completeness, or usefulness of any information, apparatus, product, or process disclosed, or represents that its use would not infringe privately owned rights. Reference herein to any specific commercial product, process, or service by trade name, trademark, manufacturer, or otherwise does not necessarily constitute or imply its endorsement, recommendation, or favoring by the United States Government or any agency thereof. The views and opinions of authors expressed herein do not necessarily state or reflect those of the United States Government or any agency thereof. 


\section{DISCLAIMER}

Portions of this document may be illegible in electronic image products. Images are produced from the best available original document. 
Library of Congress Cataloging in Publication Data

Carter, Leland Lavele, 1937-

Particle-transport simulation with the Monte Carlo method.

(ERDA critical review series)

"TID-26607."

Includes bibliographical references and index.

1. Particles (Nuclear physics) - Data processing. 2. Particles (Nuclear physics) - Mathematical models. 3. Neutron-transport theory.

5. Monte Carlo method. 1. Cashwell, E. D., joint author.

1I. Title. III. Series: United States. Energy Research and Development Administration. ERDA critical review series.
QC793.47.E4C37
$539.7^{\prime} 21$
$75-25993$

ISBN 0-87079-021-8

Available as TID-26607 for $\$ 5.45$ from

National Technical Information Service

U. S. Department of Commerce

Springfield, Virginia 22161

\section{ERDA Distribution Category UC-34}

Printed in the United States of America

USERDA Technical information Center, Oak Ridge, Tennessee

October 1975 


\title{
PREFACE
}

In undertaking to review the Monte Carlo method as applied to particle transport, it is inevitable that we spend more time on topics of interest to us in our work at Los Alamos Scientific Laboratory. However, we have endeavored to present a variety of material which will be of general use in solving neutron- and photon-transport problems, regardiess of their origin.

Although one may argue that our remarks are directed to an audience having widely differing backgrounds and interests, we hope that this review, in addition to providing a summary of Monte Carlo methods in transport theory, will assist the uninitiated reader in using the techniques described to solve his own problems. The knowledgeable practitioner of the Monte Carlo method can skip the more elementary exposition.

We wish to express our appreciation to various people who have helped in the preparation of this manuscript. We extend our thanks to Robert F. Pigeon, Office of Information Services, U.S. Atomic Energy Commission, for his cooperation during the time this work was being prepared and for obtaining reviews of the manuscript. We are grateful to Professor Norman McCormick for suggesting that the project be undertaken and for offering his comments on parts of the original draft. Our special thanks are offered to our colleagues, W. L. "Buck" Thompson, who suggested countless improvements in the manuscript, and to C. J. Everett, whose hand is evident in much of the material presented here.

\author{
L. L. Carter \\ and \\ E. D. Cashwell
}

Los Alamos Scientific Laboratory 
0

$\theta$ 


\section{CONTENTS}

Preface .............................

1 Introduction $\ldots \ldots \ldots \ldots \ldots \ldots$

2 Sampling Methods .................... 2

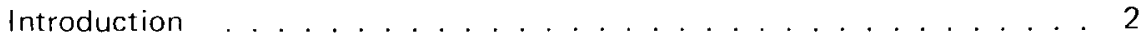

The Basic Principle ..................... . . . 2

The Rejection Technique . . . . . . . . . . . . . . . . . 8

Tables of Sampling Schemes . . . . . . . . . . . . . . . . . . 12

Importance Sampling . . . . . . . . . . . . . . . . . . 13

Splitting and Russian Roulette . . . . . . . . . . . . . . . . 16

The Exponential Transformation . . . . . . . . . . . . . . . 17

Antithetic Variates . . . . . . . . . . . . . . . . . . . . . . . . 19

Other Sampling Methods . . . . . . . . . . . . . . . . . 25

Accuracy of Monte Carlo Results . . . . . . . . . . . . . . . . 26

Central Limit Theorem . . . . . . . . . . . . . . 28

3 Mathematical Prescriptions for Simulating

Particle Transport . . . . . . . . . . . . . . . . . . 32

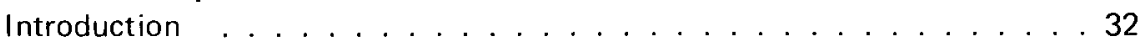

Integral-Transport-Equation Approach ............... 33

Integro-Differential Approach . . . . . . . . . . . . . . 40 40

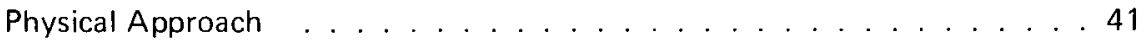


4 Mechanics of Simulating Particle Transport . . . . . . . . . . . . . . 43

Introduction ....................... . . . . 43

Geometrical Considerations . . . . . . . . . . . . . . . . . 43

Time Dependence . . . . . . . . . . . . . . . . . . . . . . . . . . . 45

Sampling the Source . . . . . . . . . . . . . . . . . 45

Attenuation and Leakage ................. . . . . . 46

Sampling the Collision Event . . . . . . . . . . . . . . . . 48

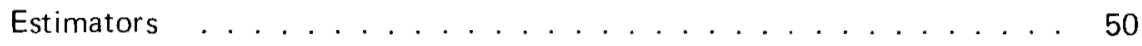

Simulation of Adjoint Equations . . . . . . . . . . . . . 55

Multigroup Monte Carlo . . . . . . . . . . . . . . . . . . . 61

$5 \quad$ Neutran Transport ....................... 65

Elastic Scattering . . . . . . . . . . . . . . . . . . . . 65

Inelastic Scattering . . . . . . . . . . . . . . . . . . . 67

Fission ........................... 70

Thermal Scattering . . . . . . . . . . . . . . . . . . 71

Criticality .......................... 75

6 Photon Transport . . . . . . . . . . . . . . . . . 79

Introduction . . . . . . . . . . . . . . . . . . . . . 79

Compton Collisions . . . . . . . . . . . . . . . . . . . 79

Incoherent Scattering . . . . . . . . . . . . . . . . . . . . . . . . . 81

Coherent Scattering . . . . . . . . . . . . . . . 83

Photoelectric Effect . . . . . . . . . . . . . . . . . . . . . 85

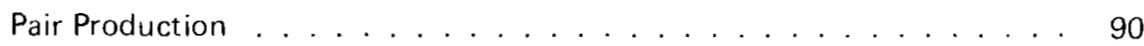

Combined Neutron and Photon Transport . . . . . . . . . . . . . . . 91

7 Literature Survey . . . . . . . . . . . . . . . . 94

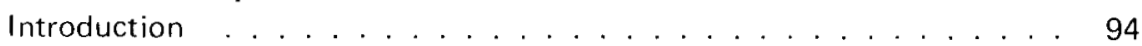

Books on Monte Carlo and Probability Theory . . . . . . . . . . . . . . . . 94

Sampling and Scoring Techniques . . . . . . . . . . . . . . . . . . 95

Biasing Methods . . . . . . . . . . . . . . . . . . . . . . . . . . . . 97

Neutron and Photon Transport . . . . . . . . . . . . . . . . . . 98

Applications of Monte Carlo in Other Areas . . . . . . . . . . . . 100

General References on the Monte Carlo Method . . . . . . . . . . . 100

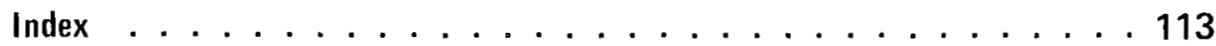


The computational speed and fast memory capacity of the modern digital computer have made practical the simulation of difficult mathematical problems with the Monte Carlo method of statistical trials. Although the Monte Carlo method is typically used to simulate a random process, it frequently is applied to problems that do not have an immediate probabilistic interpretation. Thus it has become a useful computational tool in all major areas of scientific endeavor.

Since complete treatment of the Monte Carlo method is beyond the scope of this review, we have focused attention on its application to particle-transport problems, with major emphasis on neutron and photon transport. Historically Monte Carlo has been a useful computational tool for particle-transport problems, and this is still its area of most extensive use. However, a sampling of references in other areas has been included in Chap. 7 to provide a starting point for the interested reader.

In Chap. 2 sampling techniques and the basic mathematics used in a Monte Carlo calculation are discussed. Chapters 3 to 6 present a bird's-eye view of the theory and use of Monte Carlo in solving neutron- and photon-transport problems. References are cited to enable a deeper study of each topic. 


\section{SAMPLING MEtHODS}

\section{INTRODUCTION}

The Monte Carlo method is distinguished from other techniques in numerical analysis by the use of random sampling to construct the solution of a physical or mathematical problem. A stochastic model, which may or may not be immediately obvious from the problem, is set up, and, by sampling from appropriate probability distributions, we estimate the required numerical answers to the problem by statistical means. In the treatment of particle-transport problems, which we are primarily interested in here, the probabilistic methods used may require rather sophisticated mathematical tools to justify them rigorously. The interested reader is referred to the report by Spanier ${ }^{1}$ and the book by Spanier and Gelbard. ${ }^{2}$ However, much of Monte Carlo is intuitive in nature and requires only a knowledge of elementary probability theory. In this chapter, we shall review basic sampling techniques essential to the treatment of transport problems as well as remark on the estimation of errors in these problems.

\section{THE BASIC PRINCIPLE}

Throughout our discussion we assume that we have at our disposal a supply of random numbers $\xi$, which are uniformly distributed on the interval $[0,1){ }^{*}$ There are

*We use the common mathematical definitions for real intervals; the open interval $(0,1)$ refers to the set of real numbers $\xi$ such that $0<\xi<1$, and the closed interval $[0,1]$ is the set of real numbers $\xi$ such that $0 \leqslant \xi \leqslant 1$. Similarly, $[0,1)$ is the set of real numbers $\xi$ such that $0 \leqslant \xi<1$, and $(0,1)$ denotes the set of real numbers $\xi$ such that $0<\xi \leqslant 1$. 
many schemes for generating such numbers. The most practical for machine use is to generate these "pseudorandom" numbers by some arithmetical subroutine as they are needed. The sequence of numbers used must satisfy certain properties of randomness. In addition to being uniformly distributed, an obvious requirement is that they be uncorrelated; for example, the size of the $(n+1) s t$ number should be independent of the size of the $n t h$ or, for that matter, of any of the preceding numbers. Since arithmetical schemes are almost universally used in present-day codes, it is not obvious that these requirements can be met. A very popular method for generating sequences of pseudorandom numbers is the congruential scheme of Lehmer. ${ }^{3}$ An informative discussion of the generation and testing of random numbers is given in Chap. III of a book by Knuth, ${ }^{4}$ which also includes a set of six "rules" to follow in choosing a good random-number generator. Additional references are contained in Knuth's discussion. The literature on the generation of random numbers before 1962 is summarized by Hull and Dobell, ${ }^{5}$ and a more comprehensive treatment is given by Jansson. ${ }^{6}$ In regard to the question of the reliability of random numbers, MacLaren and Marsaglia ${ }^{7,8}$ point out difficulties that can arise with congruential schemes in certain high-resolution applications. The work of Beyer ${ }^{9,10}$ is also concerned with the deficiencies of the linear congruential generators, discussing in more detail the lattice structure of $\mathrm{n}$-dimensional vectors obtained from such generators. An interesting discussion of the lattice structure of these vectors is also contained in a paper by Coveyou. ${ }^{11}$

Suppose $E_{1}, \ldots, E_{n}$ are $n$ independent, mutually exclusive events with probabilities $p_{1}, \ldots, p_{n}$, respectively, $p_{1}+\ldots+p_{n}=1$. Clearly, if a random number $\xi$, $0 \leqslant \xi<1$, is such that

$$
\mathrm{p}_{1}+\cdots+\mathrm{p}_{\mathrm{i}-1} \leqslant \xi<\mathrm{p}_{1}+\cdots+\mathrm{p}_{\mathrm{i}}
$$

then $\xi$ determines the event $E_{1}$. For example, $E_{1}, E_{2}$, and $E_{3}$ may refer to capture, elastic scattering, and inelastic scattering when a neutron collides with an atom. The probabilities are defined by $\mathrm{p}_{1}=\sigma_{c} / \sigma_{\mathrm{t}}, \mathrm{p}_{2}=\sigma_{s}^{\mathrm{e}} / \sigma_{\mathrm{t}}, \mathrm{p}_{3}=\sigma_{s}^{\mathrm{i}} / \sigma_{\mathrm{t}}$, with $\sigma_{\mathrm{t}}=\sigma_{\mathrm{c}}+\sigma_{\mathrm{s}}^{\mathrm{e}}+\sigma_{\mathrm{s}}^{\mathrm{i}}$.

This case of discrete probabilities can be illustrated graphically by assigning a variable $x$ on the interval $0 \leqslant x<n$ to the events $E_{1}, \ldots, E_{n}$ with the agreement that $i-1 \leqslant x<i$ represents the event $E_{j}$. Let us construct a probability density function $\mathrm{p}(\mathrm{x})$ by the definition

$$
\mathrm{p}(\mathrm{x})=\mathrm{p}_{\mathrm{i}}
$$

where $\mathrm{i}-1 \leqslant \mathrm{x}<\mathrm{i}$ for $\mathrm{i}=1,2, \ldots, \mathrm{n} .^{11 \mathrm{a}}$ Thus $\mathrm{p}(\mathrm{x})$ is a step function similar to that shown in Fig. 2.1. The sum of the rectangular areas depicted is clearly $\mathrm{p}_{1}+\ldots+\mathrm{p}_{\mathrm{n}}=1$. Now suppose we define the probability distribution function

$$
P(x)=\int_{0}^{x} p(t) d t \quad(0 \leqslant x<n)
$$


whose graph is shown in Fig. 2.2, a monotone increasing broken-line function such that $P(0)=0, P(n)=1$. Since $P(i)=p_{1}+\ldots+p_{i}$, we may interpret $P(x)$ to mean the probability of the inequality $x^{\prime} \leqslant x$ for $x=i, i=1,2, \ldots, n$. Moreover the equation

$$
\xi=P(x)=\int_{0}^{x} p(t) d t
$$

determines $x$ uniquely as a function of $\xi$ in such a way that, if $0 \leqslant \xi<1$ and if $\xi$ is distributed uniformly on the unit interval, then $x$ falls on the interval $i-1 \leqslant x<i$ with frequency $p_{i}$, thereby determining the event $E_{i}$.

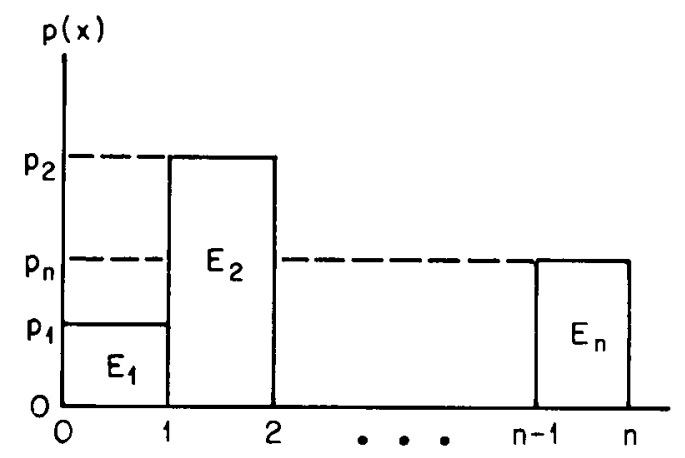

Fig. 2.1 Density function for the discrete case.

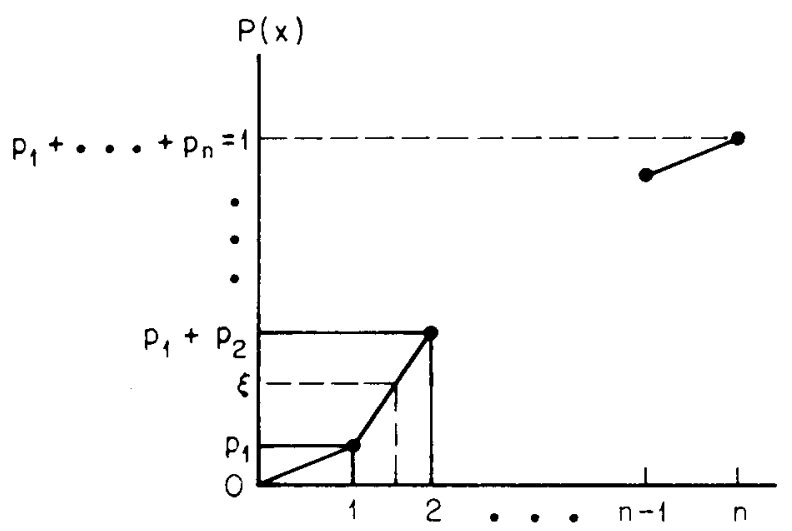

Fig. 2.2 Cumulative probability distribution function for the discrete case. 
For a continuous density function $p(x), a \leqslant x<b$, as shown in Fig. 2.3, clearly we could approximate $\mathrm{p}(\mathrm{x})$ arbitrarily closely by a step function defined on equal subdivisions of $[\mathrm{a}, \mathrm{b})$ and repeat the reasoning above to determine $\mathrm{x}, \mathrm{a} \leqslant \mathrm{x}<\mathrm{b}$, by throwing a random number $\xi$ uniformly on $[0,1)$, except the $x$ is now a continuous variable. This argument leads us to state the fundamental principle for the continuous case: If $\mathrm{p}(\mathrm{x})$ is a probability density function on the interval $\mathrm{a} \leqslant \mathrm{x}<\mathrm{b}$, then

$$
\xi=P(x)=\int_{a}^{x} p(t) d t
$$

determines $x$ uniquely as a function of $\xi$ (Fig. 2.4). Moreover, if $\xi$ is uniformly distributed on $0<\xi<1$, then $x$ falls with frequency $p(x) d x$ in the interval $(\mathrm{x}, \mathrm{x}+\mathrm{dx})$.

Let us give a couple of examples to illustrate the basic principle. Suppose we want to sample the distance to collision of a particle. The probability of a first collision between $l$ and $l+\mathrm{d} l$ along its line of flight is given by

$$
\mathrm{p}(l) \mathrm{d} l=\mathrm{e}^{-\Sigma_{\mathrm{t}} l} \Sigma_{\mathrm{t}} \mathrm{d} l
$$

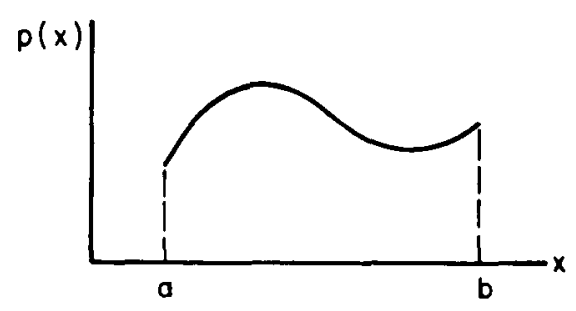

Fig. 2.3 Density function for the continuous case.

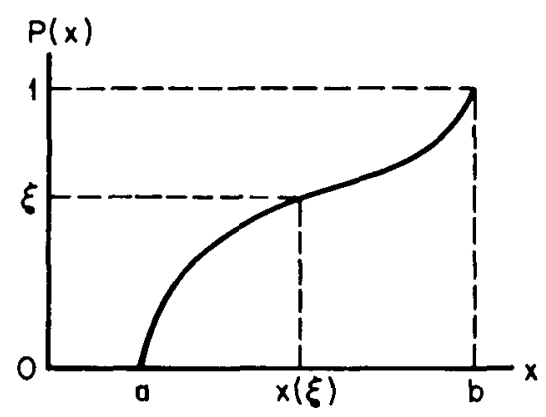

Fig. 2.4 Distribution function for the continuous case. 
where $\Sigma_{t}$ is the macroscopic total cross section of the medium and is interpreted as the probability per unit length of a collision. Setting

$$
\xi=\int_{0}^{l} \mathrm{e}^{-\Sigma_{\mathrm{t}} \mathrm{s}} \Sigma_{\mathrm{t}} \mathrm{ds}=1-\mathrm{e}^{-\Sigma_{\mathrm{t}} l}
$$

it follows that

$$
l=-\frac{1}{\Sigma_{t}} \ln (1-\xi)
$$

But, since $1-\xi$ is distributed in the same manner as $\xi$ and hence may be replaced by $\xi$, we obtain the well-known expression for the distance to collision,

$$
l=-\frac{1}{\Sigma_{\mathrm{t}}} \ln \xi
$$

Another quite different example is obtained if we wish to sample from an isotropic (spherically symmetric) distribution. That is, each element of solid angle receives the same contribution, $\mathrm{d} \Omega / 4 \pi$. One way of sampling from this function is to write it in spherical coordinates,

$$
\frac{\mathrm{d} \Omega}{4 \pi}=\frac{\sin \theta \mathrm{d} \theta}{2} \frac{\mathrm{d} \varphi}{2 \pi}
$$

where we have written the density function in the form $\mathrm{p}(\theta, \varphi)=\mathrm{p}_{1}(\theta) \mathrm{p}_{2}(\varphi)$. Thus $\theta$ and $\varphi$ are independent random variables and can be sampled separately. Setting

$$
\begin{aligned}
\xi_{1} & =\frac{1}{2} \int_{-1}^{\mu} \mathrm{d} \mu \quad(\mu=\cos \theta) \\
& =\frac{1}{2}(\mu+1) \\
\xi_{2} & =\frac{1}{2 \pi} \int_{0}^{\varphi} \mathrm{d} \varphi \\
& =\frac{\varphi}{2 \pi}
\end{aligned}
$$


we obtain

$$
\begin{aligned}
\cos \theta & \equiv \mu=2 \xi_{1}-1 \\
\varphi & =2 \pi \xi_{2}
\end{aligned}
$$

Using $\sin \theta=\left(1-\cos ^{2} \theta\right)^{1 / 2}$,

$$
\begin{aligned}
& \Omega_{\mathrm{x}}=\sin \theta \cos \varphi \\
& \Omega_{\mathrm{y}}=\sin \theta \sin \varphi \\
& \Omega_{\mathrm{z}}=\cos \theta
\end{aligned}
$$

are the direction cosines of the new direction. Later we will show how to sample directly for $\sin \varphi$ and $\cos \varphi$, rather than to evaluate the sine and $\operatorname{cosine}$ of the angle $\varphi$.

In many applications the solution of Eq. 2.5,

$$
\xi=P(x)=\int_{a}^{x} p(t) d t
$$

for $\mathrm{x}$ in terms of $\xi$ involves difficult implicit problems. An iteration method, such as the Newton-Raphson scheme, may be used to invert the relation $\xi=P(x)$, when $P(x)$ is obtained in closed analytic form.

A simple but fast method, applicable in all cases, involves subdividing the interval [a,b] and storing accurate values of $\mathrm{P}\left(\mathrm{x}_{\mathrm{i}}\right) \equiv \mathrm{P}_{\mathrm{i}}$ at the points of subdivision $\mathrm{x}_{\mathrm{o}}=\mathrm{a}<\mathrm{x}_{1}<\ldots<\mathrm{x}_{\mathrm{n}}=\mathrm{b}$. Using the method described above for discrete probabilities to determine the subinterval $\left(\mathrm{x}_{\mathrm{i}-1}, \mathrm{x}_{\mathrm{i}}\right)$ on which $\mathrm{x}$ falls, we obtain the sample value of $x$ on this interval by interpolation. If $i$ is the first value of the index for which $\xi-P_{i}$ is negative and if we use linear interpolation to determine $x$, then

$$
x=x_{i}-\frac{P_{i}-\xi}{P_{i}-P_{i-1}}\left(x_{i}-x_{i-1}\right)
$$

In some applications the values of $\mathrm{P}_{\mathrm{i}}$ will have to be obtained by numerical integration. Owing to its speed and versatility, this method is used extensively in transport codes. Equation 2.14, which fits the curve of $[x, P(x)]$ with straight lines between successive points $\left(\mathrm{x}_{\mathrm{i}^{-1}}, \mathrm{P}_{\mathrm{i}^{-}-1}\right)$ and $\left(\mathrm{x}_{\mathrm{i}}, \mathrm{P}_{\mathrm{i}}\right)$, distributes $\mathrm{x}$ uniformly on the interval $\left(\mathrm{x}_{\mathrm{i}^{-}}, \mathrm{x}_{\mathrm{i}}\right)$ and is strictly valid only when $p(x)$ is a step function. Greater accuracy may sometimes be obtained by passing through successive pairs of points on the curve of the distribution function polynominals of second or higher degree. (See Chap. 1 of Ref. 11a.) 


\section{THE REJECTION TECHNIQUE}

A method often convenient for sampling from a density function $p(x)$, $a \leqslant x \leqslant b$, is the rejection method shown in Fig. 2.5. It is frequently favored when $p(x)$ is readily computable and storage space is at a premium. Suppose $p(x)$ is bounded, and let us define

$$
\begin{aligned}
& \mathrm{p}^{*}(\mathrm{x})=\frac{\mathrm{p}(\mathrm{x})}{\operatorname{Sup} \mathrm{p}(\mathrm{x})} \\
& \int_{\mathrm{a}}^{\mathrm{b}} \mathrm{p}(\mathrm{x}) \mathrm{dx}=1
\end{aligned}
$$

where $a \leqslant x \leqslant b$. Let us select a pair of random numbers $(\xi, \eta)$ and define $x^{\prime}=a+$ $\xi(b-a)$. If $p^{*}\left(x^{\prime}\right)>\eta$, we accept $x^{\prime}$ as our sample value of $x$; otherwise we reject $x^{\prime}$ and repeat the procedure. The points $\left(\mathrm{x}^{\prime}, \dot{\eta}\right)$ are uniformly distributed in the area below the curve and above the $x$ axis. Hence it is geometrically obvious that in many trials the fraction of points $x^{\prime}$ retained between $x$ and $x+d x$ will be approximately the ratio of the areas

$$
\frac{p^{*}(x) d x}{\int_{a}^{b} p^{*}(x) d x}=\frac{p(x) d x}{\int_{a}^{b} p(x) d x}=p(x) d x
$$

This may also be seen in a more formal and rigorous manner. The probability density function of $\mathrm{x}$ is the conditional density of $\mathrm{x}$, given that $\eta<\mathrm{p}^{*}(\mathrm{x})$, and is denoted by $\mathrm{q}\left[\mathrm{x} \mid \eta<\mathrm{p}^{*}(\mathrm{x})\right]$. If we denote the joint density of $\mathrm{x}$ and $\eta$ by $\mathrm{q}(\mathrm{x}, \eta)$, where $\mathrm{q}(\mathrm{x}, \eta)=1 /(\mathrm{b}-\mathrm{a})$ in the rectangle and is 0 elsewhere, then

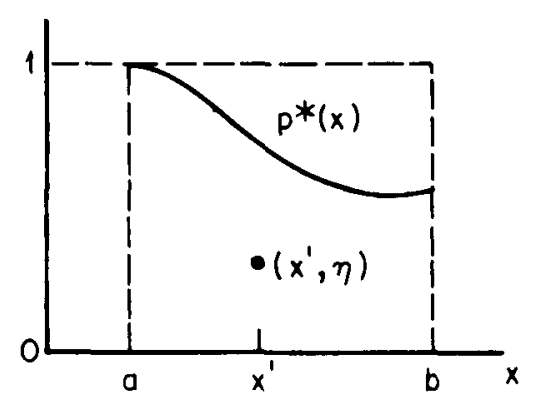

Fig. 2.5 The rejection technique. 


$$
\begin{aligned}
\mathrm{q}\left[\mathrm{x} \mid \eta<\mathrm{p}^{*}(\mathrm{x})\right] & =\frac{[1 /(\mathrm{b}-\mathrm{a})] \int_{0}^{\mathrm{p} *(\mathrm{x})} \mathrm{d} \eta}{[1 /(\mathrm{b}-\mathrm{a})] \int_{\mathrm{a}}^{\mathrm{b}} \mathrm{dx} \int_{0}^{\mathrm{p} *(\mathrm{x})} \mathrm{d} \eta} \\
& =\frac{\mathrm{p}^{*}(\mathrm{x})}{\int_{\mathrm{a}}^{\mathrm{b}} \mathrm{p}^{*}(\mathrm{x}) \mathrm{dx}}=\mathrm{p}(\mathrm{x})
\end{aligned}
$$

The efficiency of a rejection method is defined as the ratio of the number of values of $x^{\prime}$ accepted to the total number selected, and this is clearly the ratio of the area under the curve to the total area of the rectangle. That is, if $\operatorname{Sup} p(x)=A$, then

$$
\text { Efficiency }=\frac{\int_{a}^{b} p^{*}(x) d x}{b-a}=\frac{\int_{a}^{b} p(x) d x}{A(b-a)}=\frac{1}{A(b-a)}
$$

This number, which is obviously less than 1 , determines to a large extent whether a given rejection method may be feasible. Although usually convenient to use, the rejection technique may prove to be costly of machine time if the efficiency is low. Many commonly used routines that use a rejection have an efficiency considerably above $1 / 2$.

Let us consider a generalization of this method which is in common use. In fact, we shall use this device in discussing photon transport in Chap. 6. Suppose we have a probability density function $\mathrm{p}(\mathrm{x})$ on the interval $[\mathrm{a}, \mathrm{b}]$ which has the form

$$
p(x)=C g(x) f(x)
$$

Here $f(x)$ is itself a density function on $[a, b]$; without loss of generality, $g(x)$ may be bounded, $0 \leqslant g(x) \leqslant 1$; and $C$ is a normalization constant. Now we choose a sample $\mathrm{x}^{\prime}$ from the density $\mathrm{f}(\mathrm{x}), \mathrm{a} \leqslant \mathrm{x}^{\prime} \leqslant \mathrm{b}$. If a random number $\xi$ is less than $\mathrm{g}\left(\mathrm{x}^{\prime}\right), \mathrm{x}^{\prime}$ is retained as our sample from $\mathrm{f}(\mathrm{x})$; otherwise $\mathrm{x}^{\prime}$ is rejected, and we repeat the procedure. The conditional density of $\mathrm{x}$, given that $\xi<\mathrm{g}(\mathrm{x})$, is

$$
\begin{aligned}
q[x \mid \xi<g(x)] & =\frac{f(x) \int_{0}^{g(x)} d \xi}{\int_{a}^{b} f(x) d x \int_{0}^{g(x)} d \xi} \\
& =\frac{f(x) g(x)}{\int_{a}^{b} f(x) g(x) d x} \\
& =C f(x) g(x)
\end{aligned}
$$


We can show that this rejection scheme yields values with density $p(x)$ in a different manner, one which is patterned after the procedure actually followed in sampling with this method. Let us first compute the probability that the first trial in the process is successful, i.e., the value of $x$ is not rejected. The probability that $x$ is chosen between $x$ and $x+d x$ is $f(x) d x$, and then the probability that the assignment is ratified is $g(x)$, leading to the probability of $x$ in the interval $(x, x+d x)$ on the first trial given by $g(x) f(x) d x$. However, the probability of rejection on the first trial is easily seen to be

$$
\int_{a}^{b}[1-g(x)] f(x) d x=1-C^{-1}
$$

Thus the probability that $x$ will be chosen in the interval $(x, x+d x)$ on the second trial is $\left(1-C^{-1}\right) g(x) f(x) d x$. Continuing the argument through the countable sequence of trials, we obtain for the probability that $x$ will be chosen in the interval $(\mathrm{x}, \mathrm{x}+\mathrm{dx})$ the expression

$$
\sum_{k=0}^{\infty} g(x) f(x) d x\left(1-C^{-1}\right)^{k}=C g(x) f(x) d x=p(x) d x
$$

(It is observed that

$$
C=\frac{1}{\int_{a}^{b} g(x) f(x) d x}>1
$$

since

$$
\int_{a}^{b} g(x) f(x) d x<\int_{a}^{b} f(x) d x=1
$$

therefore $\left|1-\mathrm{C}^{-1}\right|<1$.)

Various other generalizations of this method appear in the literature. ${ }^{2,12}$ Let us return to an application used widely in computer codes, evaluation of $\cos \varphi$ and $\sin \varphi$, where $\varphi=2 \pi \xi$ is a uniformly distributed angle on $(0,2 \pi)$. As seen earlier, these functions arise in determining an isotropic distribution of particles, and, more generally, they appear in the process of computing a new direction of motion from an arbitrary scattering function. One can justify the rejection procedure used by appealing to a multidimensional generalization ${ }^{2}$ of the one-dimensional process described above. However, a geometric argument makes the sampling procedure transparent.

Consider Fig. 2.6, which portrays the unit circle inscribed in a square of side length 2. Choosing pairs of random numbers $\left(\xi_{1}, \xi_{2}\right)$ with $0 \leqslant \xi_{1} \leqslant 1,0 \leqslant \xi_{2} \leqslant 1$ and computing $x_{1}=2 \xi_{1}-1, x_{2}=2 \xi_{2}-1$, we see that the points $\left(\mathrm{x}_{1}, \mathrm{x}_{2}\right)$ are uniformly distributed in the square. If we retain those points inside the circle and reject those outside, it follows that each element of angle 


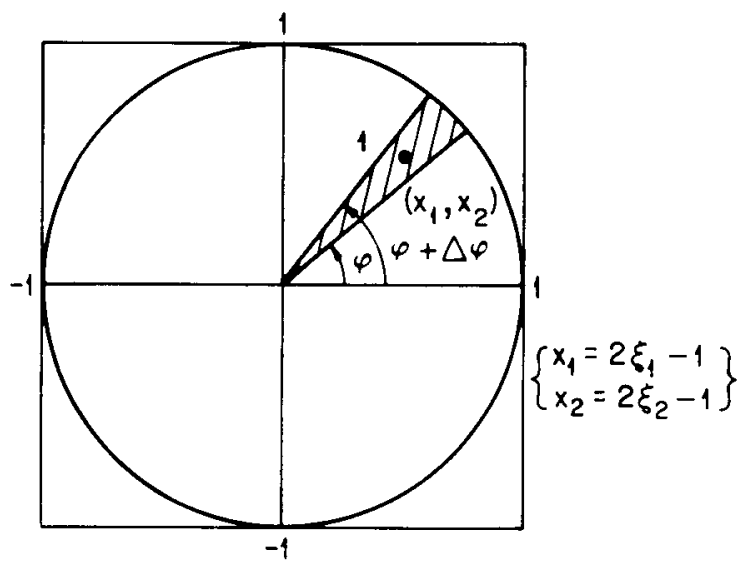

Fig. 2.6 Rejection for $\cos \varphi$ and $\sin \varphi$.

$\Delta \varphi$ will receive the same number of admissible points. Thus, if each retained point $\left(\mathrm{x}_{1}, \mathrm{x}_{2}\right)$ determines a value of $\varphi$, then $\varphi$ is sampled uniformly between 0 and $2 \pi$. Further, each point that is retained determines the functions

$$
\begin{aligned}
& \cos \varphi=\frac{x_{1}}{\left(x_{1}^{2}+x_{2}^{2}\right)^{1 / 2}} \\
& \sin \varphi=\frac{x_{2}}{\left(x_{1}^{2}+x_{2}^{2}\right)^{1 / 2}}
\end{aligned}
$$

Use of the double-angle formulas from trigonometry leads to the relations

$$
\begin{aligned}
& \cos \varphi=\frac{x_{1}^{2}-x_{2}^{2}}{x_{1}^{2}+x_{2}^{2}} \\
& \sin \varphi=\frac{2 x_{1} x_{2}}{x_{1}^{2}+x_{2}^{2}}
\end{aligned}
$$

which avoids the use of the square root.

The efficiency of this process is the ratio of the area of the circle to that of the square, and hence is $\pi / 4$. An acceptable value of the efficiency accounts for the extensive use of the above device for sampling these functions. 


\section{TABLES OF SAMPLING SCHEMES}

Many of the commonly occurring density functions in neturon- and photon-transport problems have simple and elegant schemes for sampling from them. For example, a very important density function in neutron transport is the normalized fission spectrum, which may be approximated by

$$
X(E)=\frac{2}{\pi^{1 / 2}} \frac{1}{T^{3 / 2}} E^{1 / 2} e^{-E / T}
$$

Let $\xi_{\mathrm{i}}$ represent a random number on the unit interval. Then this function may be sampled ${ }^{13}$ by choosing three random numbers $\xi_{0}, \xi_{1}$, and $\xi_{2}$, and then

$$
E=T\left[-\ln \xi_{1}-\left(\ln \xi_{2}\right) \cos ^{2}\left(\frac{\pi}{2} \xi_{0}\right)\right]
$$

Similarly, suppose we want to sample ${ }^{13}$ for the frequency $\nu$ from the normalized blackbody distribution

$$
\mathrm{b}_{\nu}=\frac{15}{\pi^{4}} \frac{\mathrm{x}^{3}}{\mathrm{e}^{\mathrm{x}}-1}
$$

where $\nu=(k T / h) x$. Set

$$
\mathrm{L}=\min \left\{l ; \sum_{1}^{l} \mathrm{j}^{-4} \geqslant \xi_{0} \frac{\pi^{4}}{90}\right\}
$$

then

$$
x=-L^{-1} \ln \left(\xi_{1} \xi_{2} \xi_{3} \xi_{4}\right)
$$

and

$$
\nu=\frac{\mathrm{kT}}{\mathrm{h}} \mathrm{x}
$$

(The notation $\{x ; F(x)\}$ means the set of all $x$ satisfying the condition $F(x)$.)

At this point in the development of Monte Carlo methods, many other examples of commonly occurring density functions can be sampled by clever and efficient schemes. The earliest compilation of such devices was made by Kahn ${ }^{14}$ and numerous examples appear throughout the later literature. A more recent collection of many of the known cases has been made by Everett and Cashwell. ${ }^{13,13 a}$ 
Another example of a simple device, one that is described in Ref. 13, which we single out for mention here since we refer to it in Chap. 5 in connection with the discussion of elastic scattering, is the following: Suppose we wish to sample from the probability density function $\mathrm{p}(\mathrm{x})$, defined on the interval $(\mathrm{a}, \mathrm{b})$, where

$$
p(x)=\sum_{j=1}^{J} a_{j}(x)
$$

with $a_{j}(x) \geqslant 0$ and $\mathbf{J}$ finite or infinite.

If we define

$$
A_{j}=\int_{a}^{b} a_{j}(x) d x
$$

It follows that

$$
\sum_{j=1}^{J} A_{j}=1
$$

Set

$$
\mathrm{K}=\min \left\{\mathrm{k} ; \sum_{1}^{\mathrm{k}} \mathrm{A}_{\mathrm{j}} \geqslant \xi\right\}
$$

where $\xi$ is a random number on the unit interval. Then sample the density function $a_{K}(x) / A_{K}$ for $x$. It is clear that the density function $a_{j}(x) / A_{j}$ is sampled with probability $A_{j}$, and hence that a sample is chosen between $x$ and $x+d x$ with probability $\mathrm{p}(\mathrm{x}) \mathrm{dx}$.

If $p_{1}(x)$ and $p_{2}(x)$ are two density functions, this scheme provides an efficient way of sampling for the interpolated density $p(x)$, where

$$
\mathrm{p}(\mathrm{x})=\alpha_{1} \mathrm{p}_{1}(\mathrm{x})+\alpha_{2} \mathrm{p}_{2}(\mathrm{x}) \quad\left(\alpha_{\mathrm{i}} \geqslant 0, \alpha_{1}+\alpha_{2}=1\right)
$$

\section{IMPORTANCE SAMPLING}

When, in the course of obtaining an estimate of a given quantity by a Monte Carlo calculation, one samples from fictitious density functions, then one is using biasing. This distortion must be corrected for by using a weight factor to alter the contribution to the quantity being estimated. Another name applied to biasing in certain instances is "importance sampling." Importance sampling refers to sampling the most important regions of a problem-in an integral, sampling well that portion of the domain which contributes most to the value, and, in a transport problem, sampling adequately those trajectories which are likely to contribute to the functional being evaluated. 
Let us assume that we have a probability density function $p(x)$ defined on $[a, b]$ and that we wish to evaluate the mean value of a function $f(x)$ when $x$ is sampled from $\mathrm{p}(\mathrm{x})$,

$$
E(f)=\int_{a}^{b} f(x) p(x) d x
$$

Suppose that, for convenience or from need to reduce the error, we choose $\mathrm{x}$ from a different density function, $\widetilde{p}(x)$. If for each point $x_{i}$ so chosen we assign a weight $\mathrm{w}\left(\mathrm{x}_{\mathrm{i}}\right)=\mathrm{p}\left(\mathrm{x}_{\mathrm{i}}\right) / \widetilde{\mathrm{p}}\left(\mathrm{x}_{\mathrm{i}}\right)$ and if the score of the game is calculated as $\mathrm{w}\left(\mathrm{x}_{\mathrm{i}}\right) \mathrm{f}\left(\mathrm{x}_{\mathrm{j}}\right)$, we are computing the mean value of $\widetilde{f}(x)=f(x) w(x)=f(x) p(x) / \widetilde{p}(x)$,

$$
E(\tilde{f})=\int_{a}^{b} \tilde{f}(x) \widetilde{p}(x) d x=\int_{a}^{b} f(x) p(x) d x=E(f)
$$

Thus the mean of $\widetilde{f}(x)$ is naturally unchanged from the mean of $f(x)$. This is not true, in general, for the variances of the two functions, since

$$
\begin{aligned}
E\left(f^{2}\right) & =\int_{a}^{b} f^{2}(x) p(x) d x \\
E\left(\widetilde{f}^{2}\right) & =\int_{a}^{b} \widetilde{f}^{2}(x) \widetilde{p}(x) d x \\
& =\int_{a}^{b} f^{2}(x) \frac{p^{2}(x)}{\widetilde{p}^{2}(x)} \widetilde{p}(x) d x \\
& =\int_{a}^{b}\left[\frac{p(x)}{\widetilde{p}(x)}\right] f^{2}(x) p(x) d x
\end{aligned}
$$

The variance is given by $E\left(\widetilde{f}^{2}\right)-E^{2}(\widetilde{f})=E\left(\widetilde{f}^{2}\right)-E^{2}(f)$. Since this is a positive quantity, if we can choose $\widetilde{p}(x)$ such that $[p(x) / \widetilde{p}(x)]<1$ over a portion of the interval (an important portion) which contributes extensively to the second moment $E\left(f^{2}\right)$, then it appears from the relation for $E\left(\widetilde{f}^{2}\right)$ that the variance has been reduced. [Note that $\mathrm{p}(\mathrm{x}) / \widetilde{\mathrm{p}}(\mathrm{x})$ cannot be less than 1 over the entire interval, because $p(x)$ and $\widetilde{p}(x)$ are density functions and hence are normalized to unity.] The perfect density $\widetilde{p}(x)$ is given by $f(x) p(x) / E(f)$ since then

$$
E\left(\widetilde{f}^{2}\right)-E^{2}(f)=\int_{a}^{b}\left[f(x) \frac{p(x)}{\widetilde{p}(x)}-E(f)\right]^{2} \widetilde{p}(x) d x=0
$$


and hence the variance is zero. Of course, if we knew $E(f)$, we would not need to sample for it. We cannot choose $\widetilde{p}(x)$ such that $\widetilde{f}(x)=f(x) p(x) / \widetilde{p}(x)$ is constant since the constant will be $E(f)$. However, the sample mean is found as the arithmetic mean of trial values of $\widetilde{f}(x)$, and hence the sample variance will be small if one chooses $\widetilde{p}(x)$ so that $f(x) p(x) / \widetilde{p}(x)$ is approximately constant. To accomplish this, the function $\widetilde{p}(x)$ may determine many more sample values in some parts of the interval than in others. In a simple case we are led naturally to the idea of importance sampling by attempting to reduce the variance.

In a similar way one is led to the idea of importance sampling in treating transport problems by the Monte Carlo method. Here we sample trajectories or random walks in phase space. Along each of these random walks, it may be desirable to sample from fictitious density functions for points in phase space, correcting by altering the statistical weight that may be assigned to the particle in this case. One attempts to emphasize the choice of points in phase space which will lead to significant contributions to the function of interest.

Consider the integral form of the transport equation

$$
F(R)=\int K\left(R ; R^{\prime}\right) F\left(R^{\prime}\right) d R^{\prime}+S_{f}(R)
$$

where $R$ denotes the kinetic energy, direction of flight, and position of the particle; $F(R)$ is the density of particles entering collision at $R ; S_{f}(R)$ is the first-flight collision density due to extraneous sources; and $K\left(R ; R^{\prime}\right)$ is the next-flight collision density at $R$ due to a collision occurring at $R^{\prime}$. This equation is discussed in more detail in Chap. 3 . Suppose we wish to estimate the functional

$$
J=\int F(R) h(R) d R
$$

where $h(R)$ is the contribution to the desired quantity of interest due to a collision at R.

In attempting to bias the random-walk process in favor of trajectories more likely to contribute to the above functional, we might make an estimate of the expected contribution to $\mathrm{J}$ from a particle at $\mathrm{R}$. If such an importance function, $\mathrm{I}(\mathrm{R})$, is available, it can be used as a weighting function to modify the density functions that yield, at each step of the random walk, the next point $R$ in phase space. The weighting function $I(R)$ can be used to define new functions $\widetilde{S}_{f}(R)$ and $\widetilde{K}\left(R ; R^{\prime}\right)$ as follows:

$$
\begin{aligned}
& \widetilde{S}_{f}(R)=\frac{S_{f}(R) I(R)}{\int S_{f}\left(R^{\prime}\right) I\left(R^{\prime}\right) d R^{\prime}} \\
& \widetilde{K}\left(R ; R^{\prime}\right)=\frac{K\left(R ; R^{\prime}\right) I(R)}{I\left(R^{\prime}\right)}
\end{aligned}
$$

These functions are used to define the random-walk process, the weight of the particle being modified accordingly at each step. A source particle at $\mathrm{R}$ has its weight modified by the factor $S_{f}(R) / \widetilde{S_{f}}(R)$, and each particle that enters collision at $R$ from a previous 
collision at $R^{\prime}$, has its weight multiplied by the factor $K\left(R ; R^{\prime}\right) / \widetilde{K}\left(R ; R^{\prime}\right)$. For further discussion of this, the reader is referred to a review article by Goertzel and Kalos; ${ }^{12}$ in Chap. 3, however, the special case where $\mathrm{I}(\mathrm{R})$ is the solution of the adjoint equation is considered in detail. There the ideal weighting function is shown to be given by the solution to the adjoint equation

$$
F^{+}(R)=\int K\left(R^{\prime} ; R\right) F^{+}\left(R^{\prime}\right) d R^{\prime}+h(R)
$$

When this function is used for $\mathrm{I}(\mathrm{R})$, each sample trajectory yields $\mathrm{J}$ as the score. Of course, finding $F^{+}(R)$ is, in general, as difficult as obtaining $F(R)$, but these considerations indicate that an approximate solution of the adjoint equation may prove to be of real value as a weighting function.

\section{SPLITTING AND RUSSIAN ROULETTE}

One of the oldest and most successful biasing techniques, and one which is commonly used in deep-penetration problems, is that of splitting accompanied by Russian roulette. To illustrate its use, suppose we have an optically thick slab of material, say a semi-infinite slab of thickness $T, 0 \leqslant z \leqslant T$, with a source of particles prescribed either at or near the plane $z=0$. At specified plane boundaries $z=z_{i}$ in the increasing $\mathrm{z}$ direction, assume that the particle splits into $v$ identical particles, each of weight $\mathrm{W} / \nu$, where $\mathrm{W}$ is the incoming weight. Clearly, weight is preserved, and we process more ( $\nu$ times as many) particles with smaller weights. The statistics should be improved since in many shielding problems the sample size diminishes to almost nothing in the far reaches of the shield. Although $\nu$ may frequently be an integer, it need not be. All that is necessary is that the expected number of split particles be $v$; e.g., if $\mathrm{n}<\nu<\mathrm{n}+1$, a common recipe is to choose $\mathrm{n}$ particles with probability $\mathrm{n}+1-\nu$ and $\mathrm{n}+1$ particles with probability $\nu-\mathrm{n}$. Obviously many other recipes are available. If $\nu$ is not an integer, it may be preferable not to split into $\nu$ particles on the average but to split into $n+1$ particles with weights $W /(n+1)$. However, splitting is most often provided in combination with Russian roulette, and then there are advantages in preserving the value $\nu$ by which the weight is divided.

If it is desirable to split particles when they penetrate deeper into a shield, or, in general, when they enter a more important region of the problem, then it is usually sound practice to decrease the number of particles followed when they enter a less desirable region: This can be achieved by Russian roulette. In our example, if a particle proceeds across the boundary $z=z_{i}$ in the direction of decreasing $z$, Russian roulette allows the particle to survive with probability $\nu^{-1}$ and its weight to be increased by a factor $\nu$. The particle is killed with probability $1-\nu^{-1}$.

In complex geometries each cell or geometric region of the problem can be assigned an importance $I$. Then, when a particle enters cell $n+1$ from cell $n$, the ratio $I_{n+1} / I_{n}$ is examined. If $I_{n+1} / I_{n} \geqslant 1$, so that the particle is entering a cell of greater 
importance, then the particle is split into $v=I_{n+1} / I_{n}$ identical particles, each of weight $\mathrm{W} \nu^{-1}$, where $\mathrm{W}$ is the incoming weight. If $\nu=\mathrm{I}_{\mathrm{n}+1} / \mathrm{I}_{\mathrm{n}}<1$, then Russian roulette is played, and the particle survives with probability $\nu$ with weight $\mathrm{W} \nu^{-1}$. Some variation of this scheme is built into most Monte Carlo codes ${ }^{15}$ in general use.

When splitting and Russian roulette are combined as we have described, then the particles that are in a region of importance I will tend to have the same weights-if these are the only processes modifying the weight of a particle, the weights will be identical-and this is usually advantageous in computing the variance of a scored quantity.

The device of assigning an importance to a cell in the problem can be generalized to make this quantity a function of other variables in the problem. For example, by this means the splitting and Russian roulette can be made to depend on the energy of the particle.

Splitting, with Russian roulette, is undoubtedly the most widely used variance-reduction technique. Simple to use, it is also hard to abuse-it usually saves machine time. With a shielding problem, we find it efficient to maintain roughly the same sample size (by splitting) as the shield is penetrated. Although the recipe depends on the material composition, this means that the particles should be split approximately 2 for 1 at boundaries spaced a distance of $\lambda$ (mean free path) apart. Sample calculations may be advisable in setting up the splitting parameters for maximum efficiency, although rough estimates usually pay dividends in reducing the amount of machine time necessary for a given error. In calculations on the penetration of thick shields ( $\sim 10$ to $20 \lambda$ ), savings in time of several orders of magnitude have been obtained by efficient use of splitting and Russian roulette.

In the slab problem considered above, the process of splitting, accompanied by Russian roulette, may be thought of as an example of importance sampling where the transport kernel is modified. In the limit of infinitely many splitting boundaries, it is a special case of the exponential transformation, ${ }^{2,12}$ which we consider next.

\section{THE EXPONENTIAL TRANSFORMATION}

In attempting to improve on straight model sampling when applied to deep-penetration problems, we find that the devices of splitting and Russian roulette, discussed in the previous section, appeal to the intuition. It appears obvious that one should artificially increase the number of particle histories in important regions (with suitable modification of weights, of course) while decreasing the sample size in unimportant regions. Another way in which this might be accomplished would be to artificially increase the distance between collisions in the desired direction, say, that of increasing penetration of a shield, while at the same time decrease the collision distance in the opposite direction. Such a method of importance sampling is effected by the exponential transformation.

In order to discuss the exponential transformation, suppose we again consider the semi-infinite shield of thickness $T, 0 \leqslant z \leqslant T$, with a source confined to small $z$. 
Since the flux of particles will fall off approximately exponentially in the positive $z$ direction, it appears that a good guess for the importance function discussed earlier will be given by

$$
I(z)=e^{\alpha z}
$$

The transformation ${ }^{12}$ of the transport equation which results from the modifications of the source and kernel through the use of this importance function is called the exponential transformation. Omitting the mathematical details in transforming the transport equation, the net effect of using this importance function is to modify the transport kernel, in that the macroscopic total cross section, $\Sigma_{t}$, is replaced by $\Sigma_{t}-\alpha w$, where $w$ is the direction cosine of the line of flight of the particle with the $\mathrm{z}$-axis. Thus in the transport process for an arbitrary direction, the probability of having a first collision at a distance between $s$ and $s+d s$ is given by

$$
\left(\Sigma_{t}-\alpha w\right) e^{-\left(\Sigma_{t}-\alpha w\right) s} d s
$$

In the analog simulation this probability should be

$$
\Sigma_{t} e^{-\Sigma_{t} s} d s
$$

Hence, if we use the fictitious $\Sigma_{t}-\alpha w$ for sampling the random walk, we should multiply the weight of the particle entering collision by

$$
\frac{\Sigma_{\mathrm{t}}}{\Sigma_{\mathrm{t}}-\alpha \mathrm{w}} \mathrm{e}^{-\alpha w s}
$$

or, since ws $=\Delta z$, by $\frac{\Sigma_{t}}{\Sigma_{t}-\alpha w} e^{-\alpha \Delta z}$.

In Monte Carlo codes with complex geometry and many materials, it is frequently convenient to adjust the weight of the particle as we go. If we proceed in this manner, the scattering kernel is unchanged; i.e., the scattering process is unaltered by this transformation.

Usually $\alpha$ is chosen so that $\Sigma_{t}-\alpha w>0$. If so, the fictitious total cross section will be smaller for positive $w$ and larger for negative $w$, leading to longer flight paths between collisions in the direction of increasing penetration of the shield and shorter flight paths in the opposite direction.

If $\Sigma_{\mathrm{t}}-\alpha \mathrm{w}<0$, one may add a term $\Sigma_{t}^{\prime} \varphi$ to both sides of the integrodifferential transport equation written in terms of the flux, $\varphi$, such that $\left(\Sigma_{\mathrm{t}}-\alpha \mathrm{w}+\Sigma_{\mathrm{t}}^{\prime}\right)>0$ for $\alpha$ and all values of $\mathrm{w}$. The term $\Sigma_{\mathrm{t}}^{\prime} \varphi$ can be regarded as a new process, whose cross section is $\Sigma_{t}^{\prime}$, in which particles continue undeflected with all parameters unchanged. 
The exponential transformation and its use in solving deep-penetration problems is discussed by Leimdorfer ${ }^{16,17}$ and Levitt. ${ }^{18}$

\section{ANTITHETIC VARIATES}

The antithetic method of reducing the variance by a modification of the scoring scheme was proposed as an important Monte Carlo technique by Hammersley and Morton ${ }^{19}$ in 1956 . The method was developed further in a series of papers, ${ }^{20-23}$ and shorter treatments are to be found in the books by Hammersley and Handscomb ${ }^{24}$ and by Spanier and Gelbard. ${ }^{2}$ We offer a brief summary here.

Given random variables $X, Y$ with means $\bar{X}, \bar{Y}$, respectively, then the covariance of $\mathrm{X}$ and $\mathrm{Y}$ is defined as

$$
\operatorname{cov}(X, Y)=E[(X-\bar{X})(Y-\bar{Y})]
$$

where $E[Z]$ stands for the expected value of $Z$. If $X$ and $Y$ are independent random variables, $\operatorname{cov}(X, Y)=0$, although the converse does not necessarily hold. We may define the correlation coefficient of $\mathrm{X}$ and $\mathrm{Y}$ as

$$
\rho(X, Y)=\frac{\operatorname{cov}(X, Y)}{[(\operatorname{var} X)(\operatorname{var} Y)]^{1 / 2}}
$$

It follows from the Cauchy-Schwarz inequality that $\rho$ has a value between -1 and +1 . If $\rho=0$, the two random variables are said to be uncorrelated, and therefore independent random variables are uncorrelated. It is for this reason that $\rho(X, Y)$ is frequently used as a measure of the dependence between $X$ and $Y$. If $\rho(X, Y)>0$, the variables are said to be positively correlated; if $\rho(X, Y)<0$, they are negatively correlated.

In the method of antithetic variates, given the estimator $t$ with expectation $\theta$, we seek a second estimator $t^{*}$ having the same expectation as $t$ but possessing a strong negative correlation with $\mathrm{t}$. Then $\left(\mathrm{t}+\mathrm{t}^{*}\right) / 2$ will be an unbiased estimator of $\theta$, with variance

$$
\operatorname{var}\left[\frac{\left(\mathrm{t}+\mathrm{t}^{*}\right)}{2}\right]=\frac{\operatorname{var} \mathrm{t}}{4}+\frac{\operatorname{var} \mathrm{t}^{*}}{4}+\frac{\operatorname{cov}\left(\mathrm{t}, \mathrm{t}^{*}\right)}{2}
$$

If $\operatorname{cov}\left(t, t^{*}\right)<0$, then it may be true that

$$
\operatorname{var}\left[\frac{\left(\mathrm{t}+\mathrm{t}^{*}\right)}{2}\right]<\operatorname{var} \mathrm{t}
$$


For example, suppose we wish to evaluate

$$
\langle G\rangle=\int_{0}^{1} g(x) d x
$$

The usual Monte Carlo procedure is to select a random variable $\xi$ uniformly on the unit interval and evaluate

$$
\overline{\mathrm{G}}=\frac{1}{\mathrm{n}} \sum_{\mathrm{i}=1}^{\mathrm{n}} \mathrm{g}\left(\xi_{\mathrm{i}}\right)
$$

$\overline{\mathrm{G}}$ is taken as the estimate of $\langle\mathrm{G}\rangle$. Consider instead the scoring function

$$
\begin{aligned}
& \widetilde{g}(x)=\alpha g(\alpha x)+(1-\alpha) g[1-(1-\alpha) x] \quad 0 \leqslant \alpha \leqslant 1 \\
& \int_{0}^{1} \widetilde{g}(x) d x=\alpha \int_{0}^{1} g(\alpha x) d x+(1-\alpha) \int_{0}^{1} g[1-(1-\alpha) x] d x \\
&=\int_{0}^{\alpha} g(t) d t+\int_{\alpha}^{1} g(t) d t \\
&=\int_{0}^{1} g(t) d t=\langle G\rangle
\end{aligned}
$$

Thus the expectation of $\widetilde{g}(x)$ is $\langle G\rangle$. On the other hand, if

$$
\int_{0}^{1} \widetilde{g}^{2}(x) d x<\int_{0}^{1} g^{2}(x) d x
$$

the variance is reduced.

We may observe that, regardless of the function $g(x)$,

$$
\int_{0}^{1} \widetilde{g}^{2}(x) d x \leqslant \int_{0}^{1} g^{2}(x) d x
$$

and hence the variance of $\widetilde{g}(\mathrm{x})$,

$$
\int_{0}^{1} \widetilde{g}^{2}(x) d x-\langle G\rangle^{2}
$$

is never larger than the variance of $\mathrm{g}(\mathrm{x})$,

$$
\int_{0}^{1} g^{2}(x) d x-\langle G\rangle^{2}
$$


To see this, let us compute

$$
\begin{aligned}
\int_{0}^{1} \widetilde{g}^{2}(x) d x=\alpha^{2} \int_{0}^{1} g^{2}(\alpha x) d x & +2 \alpha(1-\alpha) \int_{0}^{1} g(\alpha x) g[1-(1-\alpha) x] d x \\
& +(1-\alpha)^{2} \int_{0}^{1} g^{2}[1-(1-\alpha) x] d x
\end{aligned}
$$

But, by using an elementary inequality, we have

$$
2 g(\alpha x) g[1-(1-\alpha) x] \leqslant g^{2}(\alpha x)+g^{2}[1-(1-\alpha) x]
$$

with equality only if $\mathrm{g}(\alpha \mathrm{x}) \equiv \mathrm{g}[1-(1-\alpha) \mathrm{x}]$. Equality is clearly impossible for strictly monotone $\mathrm{g}(\mathrm{x})$. Substituting for the inequality and combining terms, we obtain

$$
\begin{aligned}
\int_{0}^{1} \widetilde{g}^{2}(x) d x & \leqslant \alpha \int_{0}^{1} g^{2}(\alpha x) d x+(1-\alpha) \int_{0}^{1} g^{2}[1-(1-\alpha) x] d x \\
& =\int_{0}^{\alpha} g^{2}(t) d t+\int_{\alpha}^{1} g^{2}(t) d t \\
& =\int_{0}^{1} g^{2}(t) d t
\end{aligned}
$$

Therefore

$$
\int_{0}^{1} \widetilde{g}^{2}(x) d x \leqslant \int_{0}^{1} g^{2}(x) d x
$$

with inequality holding for strictly monotone $\mathrm{g}(\mathrm{x})$.

Suppose we consider the estimator 2,24

$$
f(x)=\frac{g(x)+g(1-x)}{2}
$$

For all practical purposes, $\mathrm{f}(\mathrm{x})$ is equivalent to choosing $\alpha=1 / 2$ in the definition of $\tilde{g}(x)$. Defining $V=\operatorname{var} g$ and $V_{1}=\operatorname{var} f$, we see from the above arguments that $V_{1} \leqslant V$. If we define

$$
f_{n}=\frac{1}{n} \sum_{i=1}^{n} f\left(\xi_{i}\right)
$$


$f_{n}$ is an unbiased estimator of $\int_{0}^{1} g(x) d x$. Also

$$
\begin{aligned}
\operatorname{var} f_{n} & =\frac{1}{n} \operatorname{var} f \\
& =\frac{1}{n}\left(\int_{0}^{1} f^{2} d x-\langle f\rangle^{2}\right) \\
& =\frac{1}{n} V_{1} \leqslant \frac{1}{n} V
\end{aligned}
$$

We shall show that, for $g(x)$ monotone, $v_{1} \leqslant v / 2$, which makes the estimator $f(x)$ much more attractive since the evaluation of $f$ is about twice as much work as the evaluation of $\mathrm{g}$.

This result is given by Spanier and Gelbard. ${ }^{2}$ The proof depends on the following lemma. Since the lemma occurs as a by-product of some work on inequalities by Everett and Cashwell, 25-27 we give a trivial proof that does not depend on the continuity of the functions.

Lemma. Let $\mathrm{g}(\mathrm{x})$ be a nonnegative monotone nondecreasing (nonincreasing) function and let $\mathrm{q}(\mathrm{x})$ be a monotone nondecreasing (nonincreasing) function with

$$
\int_{0}^{1} q(x) d x=0
$$

Then

$$
\int_{0}^{1} g(x) q(x) d x \geqslant 0
$$

Proof. Given that $\mathrm{q}(\mathrm{x})$ is a monotone nondecreasing function with

$$
\int_{0}^{1} q(x) d x=0
$$

then there exists an $x_{0}$ such that

$$
\begin{array}{ll}
\mathrm{q}(\mathrm{x}) \leqslant 0 & 0 \leqslant \mathrm{x}<\mathrm{x}_{0} \\
\mathrm{q}(\mathrm{x}) \geqslant 0 & \mathrm{x}_{0}<\mathrm{x} \leqslant 1
\end{array}
$$

Thus $\int_{0}^{x_{0}} q(x) d x \leqslant 0, \int_{x_{0}}^{1} q(x) d x \geqslant 0$, and $g(x) \geqslant 0$ everywhere on the unit interval. 
Hence

$$
\begin{aligned}
\int_{0}^{1} g(x) q(x) d x & =\int_{0}^{x_{0}} g(x) q(x) d x+\int_{x_{0}}^{1} g(x) q(x) d x \\
& \geqslant g\left(x_{0}\right) \int_{0}^{x_{0}} q(x) d x+g\left(x_{0}\right) \int_{x_{0}}^{1} q(x) d x \\
& =g\left(x_{0}\right) \int_{0}^{1} q(x) d x=0
\end{aligned}
$$

Therefore

$$
\int_{0}^{1} g(x) q(x) d x \geqq 0
$$

For nonincreasing functions the proof is similar.

Now the following result follows easily:

Theorem. If $\mathrm{g}$ is a nonnegative monotone function of $\mathrm{x}, \mathrm{v}_{1} \leqslant \mathrm{~V} / 2$.

Proof.

$$
\begin{aligned}
V_{1}= & \int_{0}^{1} f^{2}(x) d x-\langle f\rangle^{2} \\
= & \frac{1}{4} \int_{0}^{1} g^{2}(x) d x+\frac{1}{4} \int_{0}^{1} g^{2}(1-x) d x \\
& +\frac{1}{2} \int_{0}^{1} g(x) g(1-x) d x-\langle g\rangle^{2}
\end{aligned}
$$

If in the lemma above we choose

$$
q(x)=1-\frac{g(1-x)}{\int_{0}^{1} g(1-x) d x}
$$

then $\mathrm{q}$ is monotone with

$$
\int_{0}^{1} q(x) d x=0
$$


Therefore, by the lemma,

$$
\begin{aligned}
\int_{0}^{1} g(x) q(x) d x & =\int_{0}^{1} g(x) d x-\frac{\int_{0}^{1} g(x) g(1-x) d x}{\int_{0}^{1} g(1-x) d x} \\
& \geqslant 0
\end{aligned}
$$

or

$$
\int_{0}^{1} g(x) g(1-x) d x \leqslant \int_{0}^{1} g(x) d x \int_{0}^{1} g(1-x) d x
$$

Therefore

$$
\begin{aligned}
V_{1} \leqslant & \frac{1}{4} \int_{0}^{1} g^{2}(x) d x+\frac{1}{4} \int_{0}^{1} g^{2}(1-x) d x \\
& +\frac{1}{2} \int_{0}^{1} g(x) d x \int_{0}^{1} g(1-x) d x-\langle g\rangle^{2} \\
= & \frac{1}{2}\left[\int_{0}^{1} g^{2}(x) d x-\langle g\rangle^{2}\right]
\end{aligned}
$$

Hence

$$
\mathrm{V}_{1} \leqslant \frac{\mathrm{V}}{2}
$$

Where $g(x)$ is not necessarily monotone, Goertzel and $\mathrm{Kalos}^{12}$ point out that an estimator that may be effective is given by

$$
\hat{\mathrm{g}}(\mathbf{x})=\alpha \mathrm{g}(\alpha \mathbf{x})+(1-\alpha) \mathrm{g}[\alpha-(1-\alpha) \mathrm{x}]
$$

This function had previously been considered by Hammersley and Morton. ${ }^{19}$

As a simple example of the use of antithetic variates in a practical situation, Hammersley and Handscomb ${ }^{24}$ discuss the problem of calculating the multiplication rate in a sphere of fissile material, where it is assumed that fission neutrons are emitted isotropically. In estimating the eigenvalue, they chose that neutrons in pairs from fission be emitted in opposite directions and made estimates of the expected number of fissions occurring in the system in each of the two directions. These estimates are negatively correlated since, if one is too large, then the other is too low. A better estimate is then expected to be the arithmetic mean of the two.

Another example mentioned by Spanier and Gelbard ${ }^{2}$ involves the use of antithetic variates in estimating resonance integrals. Neutrons born at higher energies 
will usually contribute more heavily to the resonance integrals simply because they pass through more energies at which resonances occur. The source of resonance neutrons is sampled in such a manner that a particle born at high energy is paired with a particle born at a correspondingly low energy. These two contributions are negatively correlated, and using their mean as the value of the contribution can lead to much more efficient sampling of the resonance integrals.

\section{OTHER SAMPLING METHODS}

In closing our summary of some of the most useful methods of sampling employed in Monte Carlo, we mention two sampling schemes that are quite helpful in practice and refer the reader to the literature for a more comprehensive discussion of each.

One sampling scheme is the method of stratified sampling. ${ }^{24}$ (See the discussion of quota sampling by Goertzel and Kalos ${ }^{12}$ and the treatment of systematic source sampling by Spanier and Gelbard. ${ }^{2}$ ) In this method the sampling region is subdivided into discrete subregions, and each of these is sampled a preassigned number of times. When the subdivisions, as well as the number of sampling points in each subdivision, are chosen with a view to minimizing the variance of the stratified sampling estimator, appreciable savings in computer time can result. In any event the scheme is easy to put into practice, and even elementary attempts at stratification will usually lead to an increase in efficiency over the use of straightforward (crude) Monte Carlo.

The second sampling method, which is very important in many applications, is the technique of correlated sampling. ${ }^{2,28}$ This method of calculating differential effects is applied to the Monte Carlo sampling of two random variables that have a strong positive correlation. If the changes between the two problems under investigation are small so that one problem may be regarded as a perturbation of the other, separate Monte Carlo calculations for the two systems may be impractical. The statistical uncertainties in the estimates may mask the difference between them that is being sought. It may be possible to correlate the two problems by using a single set of particle histories. One set of histories is descriptive of the base problem, and the effect of the perturbation is calculated at each collision by weight factors that correct for the changes in the collision process. Even when a single random-walk process is impractical to use for the two problems, the pair of Monte Carlo runs can be correlated by providing each new history in the base and perturbed problems with the same initial pseudorandom number (as well as the same sequence of subsequent numbers, as far as they are needed), taking care that these initial numbers form a pseudorandom sequence. Small changes in a system can thus be effectively estimated since many of the histories in the two runs will be identical. 


\section{ACCURACY OF MONTE CARLO RESULTS}

In applying the Monte Carlo method to obtain solutions to practical problems, one must have confidence in the numerical values obtained. This usually involves an estimate of the statistical errors in these values. Quite general results from probability theory are available for this purpose.

One of these results from the theory of probability is the law of large numbers. This result states that the accuracy of an estimate of a quantity tends to improve as one averages larger and larger samples of observations of the value of the quantity. To apply this to our Monte Carlo calculations, suppose that $\mathrm{x}_{1}, \mathrm{x}_{2}, \ldots, \mathrm{x}_{\mathrm{n}}$ are sample values of the random variable $x$. If the sample mean

$$
x_{n}=\frac{1}{n} \sum_{i=1}^{n} x_{i}
$$

is formed, the law of large numbers states that the sample mean, with a probability that approaches 1 as $n$ increases to infinity, approximates the population mean (or true mean), $\mathrm{E}(\mathrm{x})$.

The law of large numbers can be demonstrated quite simply. To do this, however, we need a very general result in probability theory known as Chebyshev's inequality. Given a random variable $X$ with arbitrary distribution, but with mean $m$ and standard deviation $\sigma$, it is easy to deduce a quantitative result about the closeness of $\mathrm{X}$ to the mean $m$ in terms of the standard deviation $\sigma$, i.e.,

$$
\mathrm{P}\{|\mathrm{X}-\mathrm{m}| \leqslant \mathrm{h} \sigma\} \geqslant 1-\frac{1}{\mathrm{~h}^{2}}
$$

or

$$
\mathrm{P}\{|\mathrm{X}-\mathrm{m}|>\mathrm{h} \sigma\} \leqslant \frac{1}{\mathrm{~h}^{2}}
$$

where $P\{Z\}$ means the probability of $Z$. This is Chebyshev's inequality.

Let us consider a binomial distribution. That is, suppose we consider a random variable $x$ that takes the value 1 with probability $p$ and the value 0 with probability $1-p=q$. This corresponds to an experiment with two possible outcomes, success or failure, occurring with probabilities $\mathrm{p}$ and $\mathrm{q}$, respectively. Such an experiment is called a Bernoulli trial. Let $x_{i}$ represent the value of the random variable $x$ at the $i t h$ Bernoulli trial. Then

$$
x_{n}=\frac{1}{n} \sum_{i=1}^{n} x_{i}
$$


represents the frequency of successes in $n$ trials. It can be shown that

$$
\lim _{n \rightarrow \infty} P\left\{\left|X_{n}-p\right| \leqslant \epsilon\right\}=1
$$

or, alternatively, that

$$
\lim _{\mathrm{n} \rightarrow \infty} \mathrm{P}\left\{\left|\mathrm{X}_{\mathrm{n}}-\mathrm{p}\right|>\epsilon\right\}=0
$$

This may be proved quite simply by using Chebyshev's inequality. Since the random variable $x$ has mean $p$ and variance $p(1-p)$, it follows that $X_{n}$ has mean $p$ and variance $[\mathrm{p}(1-\mathrm{p})] / \mathrm{n}$. Applying Chebyshev's inequality with $\epsilon=\mathrm{h} \sigma$, we obtain

$$
P\left\{\left|X_{n}-p\right|>\epsilon\right\} \leqslant \frac{p(1-p)}{n \epsilon^{2}}
$$

It follows that

$$
\lim _{n \rightarrow \infty} P\left\{\left|X_{n}-p\right|>\epsilon\right\}=0
$$

regardless of the value of $p$.

Similarly, if $x_{i}$ is a random sample of a random variable $x$ with finite mean $m$ and standard deviation $\sigma$, then the arithmetic mean of the $n$ independent variables $\mathrm{x}_{1}, \ldots, \mathrm{x}_{\mathrm{n}}$ is given by Eq. 2.68. $\mathrm{X}_{\mathrm{n}}$ has mean $\mathrm{m}$ and variance $\sigma^{2} / \mathrm{n}$. Again it follows from Chebyshev's inequality that

$$
\mathrm{P}\left\{\left|\mathrm{X}_{\mathrm{n}}-\mathrm{m}\right|>\epsilon\right\} \leqslant \frac{\sigma^{2}}{\mathrm{n} \epsilon^{2}}
$$

or

$$
\lim _{n \rightarrow \infty} P\left\{\left|X_{n}-m\right|>\epsilon\right\}=0
$$

Thus the (weak) law of large numbers has been demonstrated for the case of identically distributed random variables with mean and variance finite. More general results are available in the literature, but this version is usually sufficient for Monte Carlo applications.

In applications, however, we desire more precise information about a sample mean obtained by Monte Carlo than that it converges to the true mean. Usually we require a sharper estimate of the statistical error in the sample mean than we can obtain from applying the Chebyshev inequality. For such information we have recourse to the Central Limit Theorem of the theory of probability. 


\section{CENTRAL LIMIT THEOREM}

Given $n$ independent, identically distributed random variables $x_{1}, x_{2}, \ldots, x_{n}$ with common mean $m$ and variance $\sigma^{2}$, let

$$
x_{n}=\frac{1}{n} \sum_{i=1}^{n} x_{i}
$$

Then

$$
\lim _{n \rightarrow \infty} P\left\{m+a \frac{\sigma}{(n)^{1 / 2}} \leqslant X_{n} \leqslant m+b \frac{\sigma}{(n)^{1 / 2}}\right\}=\frac{1}{(2 \pi)^{1 / 2}} \int_{a}^{b} e^{-t^{2} / 2} d t
$$

This can be written

$$
P\left\{a \leqslant \frac{X_{n}-m}{\sigma /(n)^{1 / 2}} \leqslant b\right\} \rightarrow \frac{1}{(2 \pi)^{1 / 2}} \int_{a}^{b} e^{-t^{2} / 2} d t
$$

In this form, it follows that the distribution of the sum of $n$ independent, identically distributed random variables with finite means and variances, normalized to mean 0 and variance 1 , approaches a normal distribution as $n$ takes on large values.

In a Monte Carlo calculation, quite often the random variable is a function $x(t)$, where the choice of the real variable $t$ is governed by the probability density function $p(t)$. That is, we seek to obtain the expected value of $x$,

$$
m \equiv E(x)=\int_{-\infty}^{+\infty} x(t) p(t) d t
$$

Now the variance $\sigma^{2}$, which appears in the central limit theorem, is given by

$$
\sigma^{2}=\int_{-\infty}^{+\infty}[x(t)-E(x)]^{2} p(t) d t=E\left(x^{2}\right)-E^{2}(x)
$$

The sample mean of $n$ experimental trials, $X_{n}$, is given by

$$
x_{n}=\frac{1}{n} \sum_{i=1}^{n} x\left(t_{i}\right)=\frac{1}{n} \sum_{i=1}^{n} x_{i}
$$


With these definitions the central limit theorem stated above is applicable to the quantities scored in a Monte Carlo calculation.

In a practical calculation, one should keep in mind not only that the true mean $\mathrm{m}$ is unknown but also that the variance $\sigma^{2}$ must be estimated in order to apply the central limit theorem. If we have sampled $x$, the random variable, $n$ times, we use the sample variance $\bar{\sigma}^{2}$,

$$
\begin{aligned}
\bar{\sigma}^{2} & =\frac{1}{n-1} \sum_{i=1}^{n}\left(x_{i}-x_{n}\right)^{2} \\
& =\frac{n}{n-1}\left(\frac{1}{n} \sum_{i=1}^{n} x_{i}^{2}-X_{n}^{2}\right)
\end{aligned}
$$

as our approximation to the variance $\sigma^{2}$. Thus the estimated variance of the sample mean, $\bar{\sigma}^{2} / \mathrm{n}$, is used in the application of the central limit theorem in place of $\sigma^{2} / \mathrm{n}$. In many codes $\mathrm{n}$ is assumed sufficiently large that $\sigma^{2}$ is approximated by

$$
\bar{\sigma}^{2} \cong \frac{1}{n} \sum_{i=1}^{n} x_{i}^{2}-X_{n}^{2}
$$

The sample mean $\mathrm{X}_{\mathrm{n}}$ is our Monte Carlo estimate of $\mathrm{m}$.

We have stated here the form of the central limit theorem applicable to independent, identically distributed random variables with finite means and variances because this version of the theorem is sufficient for our needs. Another useful version of this important theorem applies to independent random variables that are not identically distributed. The interested reader should consult the literature ${ }^{29,30}$ for a broader coverage of this theorem, as well as the other topics mentioned in this section.

\section{REFERENCES}

1. J. Spanier, Monte Carlo Methods and Their Application to Neutron Transport Problems, USAEC Report WAPD-195, Bettis A tomic Power Laboratory, July 1959.

2. J. Spanier and E. M. Gelbard, Monte Carlo Principles and Neutron Transport Problems, Addison-Wesley Publishing Company, Inc., Reading, Mass., 1969.

3. D. H. Lehmer, Mathematical Methods in Large-Scale Computing Units, in Proceedings of a Symposium on Large-Scale Digital Calculating Machinery, pp. 141-146, 1949; published as Ann. Comput. Lab., Harvard Univ., 26 (1951).

4. Donald E. Knuth, The Art of Computer Programming, Vol, 2, Chap. IIl, Seminumerical Algorithms, Addison-Wesley Publishing Company, Reading, Mass., 1969.

5. T. E. Hull and A. R. Dobell, Random Number Generators, SIAM (Soc. Ind. Appl, Math.) Rev., 4: 230 (1962).

6. Birger Jansson, Random Number Generators, Victor Petterson's Bokindustri Aktiebolag, Stockholm, 1966. 
7. M. Donald MacLaren and George Marsaglia, Uniform Random Generators, J. Ass. Comput. Mach. (JACM), 12: 83-89 (1965).

8. G. Marsaglia, Random Numbers Fall Mainly in the Planes, Proc. Nat. Acad. Sci., U.S.A., 61: 25-28 (1968)

9. W. A. Beyer, R. B. Roof, and Dorothy Williamson, The Lattice Structure of Multiplicative Congruential Pseudo-Random Vectors, Math. Comput., 25(114): 345-363 (1971).

10. W. A. Beyer, Lattice Structure and Reduced Bases of Random Vectors Generated by Linear Recurrences, in Applications of Number Theory to Numerical Analysis, pp. 361-370, Academic Press Inc., New York, 1972.

11. R. R. Coveyou, Random Number Generation Is Too Important To BeLeft To Chance, Stud. Appl. Math., 3: 70-111 (1970).

11a. E. D. Cashwell and C. J. Everett, A Practical Manual on the Monte Carlo Method for Random Walk Problems, Pergamon Press, Inc., New York, 1959.

12. G. Goertzel and M. H. Kalos, Monte Carlo Methods in Transport Problems, in Progress in Nuclear Energy, Physics and Mathematics, Series I, Vol. 2, pp. 315-369, Pergamon Press, Inc., New York, 1958.

13. C. J. Everett and E. D. Cashwell, A Monte Carlo Sampler, USAEC Report LA-5061-MS, Los Alamos Scientific Laboratory, 1972.

13a. C. J. Everett and E. D. Cashwell, A Second Monte Carlo Sampler, USAEC Report LA-5723-MS, Los Alamos Scientific Laboratory, 1974.

14. Herman Kahn, Applications of Monte Carlo, USAEC Report AECU-3259, Rand Corporation, 1954.

15. E. D. Cashwel1, J. R. Neergaard, W. M. Taylor, and G. D. Turner, MCN: A Neutron Monte Carlo Code, USAEC Report LA-4751, Los Alamos Scientific Laboratory, 1972.

16. M. Leimdorfer, On the Transformation of the Transport Equation for Solving Deep Penetration Problems by the Monte Carlo Method, Trans. Chalmers Univ. Technol., Gothenberg, No. 286 (1964).

17. M. Leimdorfer, A Monte Carlo Method for the Analysis of Gamma Radiation Transport from Distributed Sources in Laminated Shields, Nukleonik, 6: 58 (1964).

18. Leo B. Levitt, The Use of Self-Optimized Exponential Biasing in Obtaining Monte Carlo Estimates of Transmission Probabilities, Nucl. Sci. Eng., 31 : 500-504 (1968).

19. J. M. Hammersley and K. W. Morton, A New Monte Carlo Technique:Antithetic Variates, Proc. Cambridge Phil. Soc., 52: $449-475$ (1956).

20. J. M. Hammersley and J. G. Mauldon, General Principles of Antithetic Variates, Proc. Cambridge Phil. Soc., 52: 476-481 (1956).

21. D. C. Handscomb, Proof of the Antithetic Variates Theorem for $\mathrm{n}>2$, Proc. Cambridge Phil. Soc., 54: 300-301 (1958).

22. J. H. Halton and D. C. Handscomb, A Method for Increasing the Efficiency of Monte Carlo Integration, J. Ass. Comput. Mach., 4: $329-340$ (1957).

23. K. W. Morton, A Generalization of the Antithetic Variate Technique for Evaluating Integrals, J. Math. Phys., 36: 289-293 (1957).

24. J, M. Hammersley and D. C. Handscomb, Monte Carlo Methods, in Methuen's Monographs on Applied Probability and Statistics, Methuen and Company, Ltd., London, 1964.

25. E. D. Cashwell and C. J. Everett, The Means of Order $t$ and the Laws of Thermodynamics, Amer. Math. Mon., 74(3): 271-274 (1967).

26. E. D. Cashwell and C. J. Everett, A General Mean Value Theorem, Bull. Amer. Math. Soc., 74(6): $1135-1138$ (1968).

27. E. D. Cashwell and C. J. Everett, The Mean of a Function $x(\nu)$ Relative to a Function w( $\xi, \nu)$, Amer. Math. Mon., 76(3): 252-261 (1969).

28. C. W. Watson and E. D. Cashwell, Monte Carlo Development and Applications in the Los Alamos Nuclear Rocket Program, in Proceedings of the Fourth International Conference on 
Reactor Shielding, Paris, October 1972, Vol. 1, p. 166, O.E.C.D. Nuclear Energy Agency, Paris, 1973.

29. E. Parzen, Modern Probability Theory and Its Applications, John Wiley \& Sons, Inc., New York, 1960.

30. M. Loeve, Probability Theory, Van Nostrand Reinhold Company, New York, 1950. 


\section{MATHEMATICAL PRESCRIPTIONS FOR S SIMULATING PARTICLE TRANSPORT}

\section{INTRODUCTION}

The Monte Carlo solution of the Boltzmann transport equation differs considerably from other standard numerical techniques. There is even a difference in the definition of what constitutes a solution. A numerical solution of the transport equation usually provides a rather complete description of the flux in all of phase space. A Monte Carlo solution does not include such fine detail but instead provides information about certain specified quantities of interest, usually integral quantities, such as reaction rates in portions of phase space. It will be understood that the word "solution" is to be interpreted in this context.

A unique feature in solving transport problems by Monte Carlo is that individual particle histories are simulated. The modeling of the physical processes may be accomplished without even referring to the transport equation. This is conceptually simple for a Markov process since the simulation at any point in the particle history does not depend on how the particle happened to reach that point.

The treatment of each physical process is actually a distinct advantage of Monte Carlo since the modeling tends to be conceptually simpler than numerical methods. However, this modeling of the physical processes is often inadequate by itself. Some reasons for this inadequacy are: (1) the physical (analog) simulation may require an excessive amount of computation time, (2) special scoring techniques may be required which are difficult to derive from a purely physical viewpoint, and (3) questions may 
arise regarding a possible bias in schemes that differ from an analog scheme. Such considerations as these indicate the need for a satisfactory mathematical description of the Monte Carlo method.

The common description of particle transport is formulated in terms of the Boltzmann transport equation. The integral form of this equation provides a useful beginning point for establishing a mathematically sound Monte Carlo simulation. The integro-differential form of the transport equation is also frequently referred to in the literature; it has been particularly useful as a starting point for constructing a sampling scheme to solve the equation that is adjoint to the transport equation.

It is interesting to note that, while the physical modeling of the Markov process is conceptually simple, a more rigorous mathematical treatment of the application of the Monte Carlo method to solve particle-transport problems is not trivial. This is quite aptly demonstrated in the first few chapters of the book by Spanier and Gelbard.'

In this review a complete mathematical description of the Monte Carlo method will not be attempted; instead, some insights will be given into various ways of looking at a Monte Carlo simulation of particle transport. Specifically, the integral form of the transport equation will be used to gain some understanding of the sampling process. The integro-differential form of the transport equation will be used to obtain additional perspectives. Finally, the physical viewpoint of a Monte Carlo simulation of the Markov process is discussed.

\section{INTEGRAL-TRANSPORT-EQUATION APPROACH}

The integral form of the transport equation as a starting point for obtaining a mathematical description of a Monte Carlo simulation has been examined in a number of places in the literature. The review article of Goertzel and Kalos, ${ }^{2}$ the journal article by Coveyou, Cain, and Yost, ${ }^{3}$ a report by Spanier, ${ }^{4}$ and the book by Spanier and Gelbard $^{1}$ provide useful references on the subject for the interested reader. A brief discussion is presented here.

The integral form of the transport equation is

$$
F(R)=\int K\left(R ; R^{\prime}\right) F\left(R^{\prime}\right) d R^{\prime}+S_{f}(R)
$$

where $\mathrm{R}$ denotes the kinetic energy, direction of flight, and spatial coordinates of the particle; $F(R)$ is the collision density; $S_{f}(R)$ is the first-flight collision density due to extraneous sources; and $K\left(R ; R^{\prime}\right)$ is the next-flight collision density at $R$ due to a collision occurring at $\mathrm{R}^{\prime}$. Time-dependent problems are not being considered here, although time may be treated by using the appropriate time-dependent kernel. The kernel, $K\left(R ; R^{\prime}\right)$, is assumed to be nonnegative and of such a nature that a solution for $F(R)$ exists. For critical assemblies this usually implies that $S_{f}(R)$ is the source from one generation of fission neutrons and $K\left(R ; R^{\prime}\right)$ is the kernel obtained by treating 
fission as a terminal event so that $F(R)$ is the collision density for one fission-to-fission generation.

The unnormalized kernel $K\left(R ; R^{\prime}\right)$ may be conveniently separated into the product of a noncapture probability, a normalization factor to account for such multiplying events as $(n, 2 n)$ inelastic scattering, and a normalized kernel. Thus the kernel $K\left(R ; R^{\prime}\right)$ is expressed as

$$
\mathrm{K}\left(\mathrm{R} ; \mathrm{R}^{\prime}\right)=\left[1-\alpha\left(\mathrm{R}^{\prime}\right)\right] \eta\left(\mathrm{R}^{\prime}\right) \beta\left(\mathrm{R} ; \mathrm{R}^{\prime}\right)
$$

where $\alpha\left(R^{\prime}\right)$ is the capture probability at the collision point $R^{\prime}$. The normalization factor $\eta\left(\mathrm{R}^{\prime}\right)$ and the normalized kernel $\beta\left(\mathrm{R} ; \mathrm{R}^{\prime}\right)$ are defined in terms of the complete kernel as

$$
\eta\left(\mathrm{R}^{\prime}\right)=\frac{\int \mathrm{K}\left(\mathrm{R} ; \mathrm{R}^{\prime}\right) \mathrm{d} \mathrm{R}}{\left[1-\alpha\left(\mathrm{R}^{\prime}\right)\right]}
$$

and

$$
\beta\left(R ; R^{\prime}\right)=\frac{K\left(R ; R^{\prime}\right)}{\int K\left(R^{\prime \prime} ; R^{\prime}\right) d R^{\prime \prime}}
$$

The reason for separating $K\left(R ; R^{\prime}\right)$ into the product form of Eq. 3.2 will become apparent later.

We may express the collision density as a summation, each term consisting of a multidimensional integral that suggests a random-walk scheme for its evaluation. This multicollision expansion may be obtained by substituting for $F\left(R^{\prime}\right)$ in Eq. 3.1 the equivalent expression

$$
F\left(R^{\prime}\right)=\int K\left(R^{\prime} ; R^{\prime \prime}\right) F\left(R^{\prime \prime}\right) d R^{\prime \prime}+S_{f}\left(R^{\prime}\right)
$$

and repeating this procedure for the resulting $F$ in each subsequent integrand. The result is that $F(R)$ may be expressed as

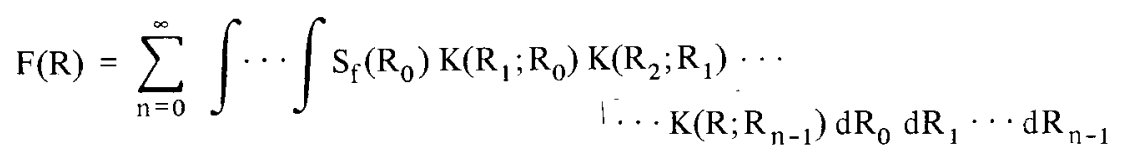

where the first term of the summation $(n=0)$ is understood to be $S_{f}(R)$.

The purpose of the Monte Carlo calculation is to compute quantities that depend functionally, usually linearly, on the collision density. We specify such a functional to be

$$
J=\int F(R) h(R) d R
$$


where $h(R)$ is the contribution that a collision at $R$ makes to the functional of interest. The multicollision expansion of Eq. 3.6 may be used to express $\mathrm{J}$ as

$$
\begin{aligned}
J=\sum_{n=0}^{\infty}\left\{\int \cdots \int S_{f}\left(R_{0}\right)\left[1-\alpha\left(R_{0}\right)\right] \beta\left(R_{1} ; R_{0}\right)\left[1-\alpha\left(R_{1}\right)\right] \beta\left(R_{2} ; R_{1}\right) \cdots\right. \\
\left.\cdots\left[1-\alpha\left(R_{n-1}\right)\right] \beta\left(R_{n} ; R_{n-1}\right) \alpha\left(R_{n}\right) W\left(R_{0}, \cdots, R_{n}\right) d R_{0} \cdots d R_{n}\right\}
\end{aligned}
$$

where $K$ has been separated into the product form of Eq. 3.2 and $W\left(R_{o}, \ldots, R_{n}\right)$ is defined as

$$
W\left(R_{0}, \cdots, R_{n}\right)=\frac{h\left(R_{n}\right)}{\alpha\left(R_{n}\right)} \prod_{i=0}^{n-1} \eta\left(R_{i}\right)
$$

The expression for $\mathrm{J}$ in Eq. 3.8 may be used to construct a Monte Carlo sampling. When you sample with the functions $S_{f}\left(R_{o}\right)$ for the initial coordinates of the particle history, ${ }^{*} \alpha\left(R_{i^{-1}}\right)$ for termination at the $\mathrm{i}$ th collision, and $\beta\left(\mathrm{R}_{\mathrm{i}} ; \mathrm{R}_{\mathrm{i}-1}\right)$ for the next collision point given that the chain continues, the quantity

$$
\begin{aligned}
\left\{\mathrm{S}_{\mathrm{f}}\left(\mathrm{R}_{0}\right)\left[1-\alpha\left(\mathrm{R}_{0}\right)\right] \beta\left(\mathrm{R}_{1} ; \mathrm{R}_{0}\right)\right. & \cdots\left[1-\alpha\left(\mathrm{R}_{\mathrm{n}-1}\right)\right] \\
& \left.\times \beta\left(\mathrm{R}_{\mathrm{n}} ; \mathrm{R}_{\mathrm{n}-1}\right) \alpha\left(\mathrm{R}_{\mathrm{n}}\right) \mathrm{dR}_{0} d \mathrm{R}_{1} \cdots \mathrm{dR}_{\mathrm{n}}\right\}
\end{aligned}
$$

is the probability that the initial coordinates for the first collision are sampled within $\mathrm{dR}_{\mathrm{o}}$ about $\mathrm{R}_{\mathrm{o}}$, the subsequent collisions of the particle fall within the phase space volume $d_{1} \ldots d R_{n}$ about $R_{1}, \ldots, R_{n}$, and the chain terminates at the $(n+1) s t$ collision. The factor $W\left(R_{0}, \ldots, R_{n}\right)$ in Eq. 3.9 is the score for such a particle in the Monte Carlo calculation. The expected value of the score is simply the product of $W\left(R_{o}, \ldots, R_{n}\right)$ with the probability chain of Eq. 3.10 integrated over phase space and summed over $\mathrm{n}$. This is equivalent to the right-hand side of Eq. 3.8; so the expected value of $W$ is $J$,

$$
E(W)=J
$$

Thus, sampling with the density functions $S_{\mathrm{f}}, \alpha$, and $\beta$ and scoring with the $\mathrm{W}$ defined by Eq. 3.9 is a Monte Carlo scheme that yields an unbiased estimate of $\mathbf{J}$.

This sampling scheme has a useful physical interpretation for many problems. For example, if the functional of interest is the capture rate in some region, then $h(R)$ is

\footnotetext{
*A normalized $\mathrm{S}_{\mathrm{f}}$ is assumed.
} 
the ratio of the capture to the total macroscopic cross section; so $h(R)=\alpha(R)$ in that region. Then, for materials in which all collisions are nonmultiplying $[\eta(R)=1]$, the sampling scheme would simulate the physical process, and the score of Eq. 3.9 at each terminal capture collision within the region of interest is unity.

There is one flaw in this derivation of the sampling scheme. The possibility exists that the capture cross section may be exactly zero in some portion of phase space which does contribute to the functional. The last-event estimator will miss such contributions, although this can easily be corrected by using a fictitious, nonzero capture cross section and adjusting the particle weight at each collision accordingly. We also expect a possible reduction in statistical errors with an estimator that scores at each collision rather than only at the termination of the chain. It is easy to show that an appropriate unbiased collision estimator for a particle that suffers termination at the $(n+1)$ st collision is given by

$\mathrm{h}\left(\mathrm{R}_{0}\right)+\mathrm{h}\left(\mathrm{R}_{1}\right) \eta\left(\mathrm{R}_{0}\right)+\mathrm{h}\left(\mathrm{R}_{2}\right) \eta\left(\mathrm{R}_{0}\right) \eta\left(\mathrm{R}_{1}\right)+\cdots+\mathrm{h}\left(\mathrm{R}_{\mathrm{n}}\right) \prod_{\mathrm{i}=0}^{\mathrm{n}-1} \eta\left(\mathrm{R}_{\mathrm{i}}\right)$

A collision estimator usually, but not always, yields a lower variance than the last-event estimator.

The last-event estimator is useful for obtaining insights into methods of reducing variances in Monte Carlo calculations. In fact, it is theoretically possible to define a sampling scheme that uses a last-event estimator and has a variance of zero, i.e., the estimate obtained with each individual source particle sample is the exact answer $\mathbf{J}$. We use the last-event estimator to show how such a sampling scheme may be derived and also to illustrate the construction of altered sampling schemes that are unbiased.

An altered sampling scheme is constructed by replacing the density functions $\mathrm{S}_{\mathrm{f}}, \alpha$, and $\beta$, which will be referred to as the analog density functions, with another set of density functions, $\widetilde{\mathrm{S}}_{\mathrm{f}}, \widetilde{\alpha}$, and $\widetilde{\beta}$. This alternate set of density functions is introduced into Eq. 3.8 in such a manner as to obtain the same value for the functional $J$. This criterion is satisfied by

$$
\begin{array}{r}
J=\sum_{n=0}^{\infty}\left\{\int \cdots \int \widetilde{S}_{f}\left(R_{0}\right)\left[1-\widetilde{\alpha}\left(R_{0}\right)\right] \widetilde{\beta}\left(R_{1} ; R_{0}\right) \cdots\left[1-\widetilde{\alpha}\left(R_{n-1}\right)\right]\right. \\
\left.\quad \times \widetilde{\beta}\left(R_{n} ; R_{n-1}\right) \widetilde{\alpha}\left(R_{n}\right) \widetilde{W}\left(R_{0}, \cdots, R_{n}\right) d R_{0} \cdots d R_{n}\right\}
\end{array}
$$

where

$$
\widetilde{W}\left(R_{0}, \cdots, R_{n}\right)=\frac{h\left(R_{n}\right) S_{f}\left(R_{0}\right)}{\widetilde{\alpha}\left(R_{n}\right) \widetilde{S}_{f}\left(R_{0}\right)} \prod_{i=0}^{n-1} \frac{\eta\left(R_{i}\right)\left[1-\alpha\left(R_{i}\right)\right] \beta\left(R_{i+1} ; R_{i}\right)}{\left[1-\widetilde{\alpha}\left(R_{i}\right)\right] \widetilde{\beta}\left(R_{i+1} ; R_{i}\right)}
$$


Equation 3.13 has the same form as Eq. 3.8; so a Monte Carlo scheme may be constructed by using the altered density functions $\widetilde{S_{f}}\left(R_{o}\right)$ to sample the initial coordinates of the particle history, $\widetilde{\alpha}\left(\mathrm{R}_{\mathrm{i}^{-}}\right)$to sample termination at the $\mathrm{i}$ th collision, and $\widetilde{\beta}\left(\mathrm{R}_{\mathrm{i}} ; \mathrm{R}_{\mathrm{i}^{-1}}\right)$ to sample for the next collision point given that the chain continues.

Certain restrictions are placed on the altered density functions to ensure that the altered Monte Carlo sampling scheme will yield the correct expected contribution to the functional $J$ from all portions of phase space. In general, an altered density function is required to satisfy the condition that it be nonzero at all points in phase space when the corresponding analog density function is nonzero. It is interesting to note the additional criterion that the altered density function be zero at all points in phase space where the analog density function is zero is not necessary. This criterion is usually included, however, since any chain that would lead to such a phase-space point would result in zero weight and hence generally degrade statistics, even though the expected value of the sampling scheme is still $J$. Nevertheless, it is sometimes convenient to use altered density functions that do not always go to zero when the analog density functions do.

The significance of evaluating the functional $\mathrm{J}$ with the altered Monte Carlo scheme is that, whereas the mean is unchanged,

$$
\mathrm{E}(\tilde{\mathrm{W}})=\mathrm{E}(\mathrm{W})=\mathrm{J}
$$

the second moments are not generally equal, i.e.,

$$
\mathrm{E}\left(\widetilde{W}^{2}\right) \neq \mathrm{E}\left(\mathrm{W}^{2}\right)
$$

The sample variance can be decreased by finding an altered sampling scheme with

$$
\mathrm{E}\left(\widetilde{\mathrm{W}}^{2}\right)<\mathrm{E}\left(\mathrm{W}^{2}\right)
$$

At this point we digress to show that there is a sampling scheme that yields zero variance, and hence each sample history predicts the exact answer $\mathbf{J}$. The existence of such a sampling scheme is easily demonstrated by referring to the equation that is adjoint to the integral transport equation,

$$
F^{+}(R)=\int K\left(R^{\prime} ; R\right) F^{+}\left(R^{\prime}\right) d R^{\prime}+h(R)
$$

The altered density functions for the zero-variance sampling scheme are defined in terms of $F^{+}(R)$ as

$$
\widetilde{S}_{f}\left(R_{0}\right)=\frac{S_{f}\left(R_{0}\right) F^{+}\left(R_{0}\right)}{\int S_{f}(R) F^{+}(R) d R}
$$




$$
\begin{gathered}
\widetilde{\alpha}\left(\mathrm{R}_{\mathrm{i}}\right)=\frac{\mathrm{h}\left(\mathrm{R}_{\mathrm{i}}\right)}{\mathrm{F}^{+}\left(\mathrm{R}_{\mathrm{i}}\right)} \\
\widetilde{\beta}\left(\mathrm{R}_{\mathrm{i}+1} ; \mathrm{R}_{\mathrm{i}}\right)=\frac{\beta\left(\mathrm{R}_{\mathrm{i}+1} ; \mathrm{R}_{\mathrm{i}}\right) \mathrm{F}^{+}\left(\mathrm{R}_{\mathrm{i}+1}\right)}{\int \beta\left(\mathrm{R} ; \mathrm{R}_{\mathrm{i}}\right) \mathrm{F}^{+}(\mathrm{R}) \mathrm{d} \mathrm{R}}
\end{gathered}
$$

These density functions may be substituted in Eq. 3.14 to obtain the scoring function for an altered scheme:

$$
\begin{aligned}
\widetilde{W}\left(R_{0}, \cdots, R_{n}\right)= & \left\{\frac{F^{+}\left(R_{n}\right)}{F^{+}\left(R_{0}\right)}\left[\int S_{f}(R) F^{+}(R) d R\right]\right. \\
& \left.\prod_{i=0}^{n-1} \frac{\eta\left(R_{i}\right)\left[1-\alpha\left(R_{i}\right)\right] F^{+}\left(R_{i}\right) \int \beta\left(R ; R_{i}\right) F^{+}(R) d R}{\left[F^{+}\left(R_{i}\right)-h\left(R_{i}\right)\right] F^{+}\left(R_{i+1}\right)}\right\}
\end{aligned}
$$

The definition of $\mathrm{K}$ in Eq. 3.2 may be used in Eq. 3.18 to obtain

$$
\eta\left(\mathrm{R}_{\mathrm{i}}\right)\left[1-\alpha\left(\mathrm{R}_{\mathrm{i}}\right)\right] \int \beta\left(\mathrm{R} ; \mathrm{R}_{\mathrm{i}}\right) \mathrm{F}^{+}(\mathrm{R}) \mathrm{dR}=\mathrm{F}^{+}\left(\mathrm{R}_{\mathrm{i}}\right)-\mathrm{h}\left(\mathrm{R}_{\mathrm{i}}\right)
$$

Therefore the expression for $\widetilde{W}$ of Eq. 3.22 simplifies to

$$
\widetilde{W}\left(R_{0}, \cdots, R_{n}\right)=\int S_{f}(R) F^{+}(R) d R
$$

i.e., a constant score independent of the history of the sample! That this constant score is indeed $J$ may be shown by multiplying Eq. 3.1 by $F^{+}(R) d R$, multiplying Eq. 3.18 by $\mathrm{F}(\mathrm{R}) \mathrm{dR}$, extracting the difference of the resulting two equations, and integrating the final equation over all of phase space.

The density functions given in Eqs. 3.19 to 3.21 can be used for a zero-variance Monte Carlo sampling scheme. However, this theoretical zero-variance sampling scheme is not directly applicable, since the construction of these density functions requires the solution of the adjoint equation for $\mathrm{F}^{+}(\mathrm{R})$. In spite of the fact that the theoretical zero-variance scheme is not used in practical problems, it does provide a useful guide for constructing schemes that are in some sense an approximation to the theoretical scheme; in addition, it is encouraging to know that there is a theoretical sampling scheme with zero variance. It should be emphasized, however, that an approximate scheme does not imply approximations in computing the functional $\mathrm{J}$. As long as the weight factor of Eq. 3.14 is used for scoring and the altered density functions satisfy the necessary and sufficient condition, the sampling scheme is still unbiased. 
A word of caution is in order now. Although the previous derivation may seem to imply that an analog scheme is easy to improve on, this is often not the case. There is the very real danger that an altered scheme will actually increase the variance (sometimes disastrously). As a rule of thumb, try to avoid sampling schemes that allow the particle weight to increase greatly, particularly without an upper bound. In many cases this difficulty can be averted by using methods that deviate less from analog simulation.

Coveyou, Cain, and Yost ${ }^{3}$ have also examined minimum variance schemes for collision estimators; i.e., each collision may contribute to the functional. They found that the use of the value function as an importance function resulted in an upper limit in the sample variance so that

$$
\frac{\text { Variance }}{\text { Mean }^{2}} \leqslant 1
$$

Here the value function is the expected value of the contribution, present and future, of a particle of unit weight.

In this discussion the integral transport equation was used to construct a Monte Carlo simulation with the collision density $F(R)$ defined as the density of particles entering a collision. The results obtained here by beginning with the transport equation for the density of particles entering a collision are correspondingly obtained by beginning with the transport equation for the density of particles leaving a collision. Pertinent relations between the two approaches are given by Spanier and Gelbard ${ }^{1}$ and by Irving. 5

The functional $\mathbf{J}$ can alternatively be computed by sampling from an equation that is adjoint to the transport equation. It can be shown that there is an altered scheme for sampling from the adjoint equation that also has zero variance. In this scheme the collision density acts as the importance function, with the result that there is symmetry between the construction of the forward and adjoint zero-variance simulations. This will be discussed further in Chap. 4 when the simulation of the adjoint equation is considered.

We have indicated how the integral transport equation can be used as a starting point to provide a sound mathematical basis for a Monte Carlo sampling. By using this approach, we were led rather naturally to an unbiased method for correcting particle weights when using an altered sampling scheme. In general, the particle weight of the analog scheme is multiplied by a correction factor at each stage of the sampling. This correction factor is the ratio of the analog density function to the corresponding density function in the altered scheme, where these density functions are evaluated at the phase-space point that is selected with the density function of the altered scheme.

The integral transport-equation viewpoint suffers from certain limitations. The multicollision expansion is awkward to work with, and, in addition, the kernel in the integral transport equation is complicated enough so that it is not easily interpretable. For these reasons, it is often preferable to work with the integro-differential form, 
which we will now consider. Both approaches are equivalent in that one can find transformations to go back and forth between the integral transport equation and the integro-differential transport equation. However, one of the equations may provide a decided advantage as a beginning point for a specific problem.

\section{INTEGRO-DIFFERENTIAL APPROACH}

The interpretation of the Monte Carlo simulation of particle transport as a solution of a multidimensional integral is not as straightforward to obtain from the integro-differential form of the transport equation. This equation can be converted to an integral postcollision transport equation, and the techniques discussed in the previous section can be applied to understand the Monte Carlo simulation. The mathematics of this transformation will not be discussed, but the interested reader may consult a report by Irving. ${ }^{5}$ For the purpose here, a few useful insights that are obtained by beginning with the transport equation in integro-differential form will be mentioned.

An important application is in the general area of biasing schemes, which typically evolve in the following manner. The particle-transport problem of interest is first stated. It is assumed that an analog or some similar simulation could be done in principle, but the calculation for the particular problem under consideration requires an excessive amount of computation time to obtain acceptable statistical-error limits. Therefore some transformation of the integro-differential transport equation is made by using information about the expected solution. A well-known example of this is the exponential transformation. ${ }^{6}$ After the transformation the new equation is examined in regard to its similarity to the original transport equation. Assuming that it has the identical form of the original equation (i.e., leakage term, attenuation term, and source term), the prescription for sampling this equation is known. The mean values of functionals do not depend on the particular transformation, but variances do. Hence it is theoretically possible to reduce the variance with the proper change of variables. Of course, there is also the very real possibility of selecting a transformation that actually increases the variance.

The integro-differential form of the transport equation is useful as a starting point for developing methods to sample from equations that are adjoint to transport equations. ${ }^{5,7-9}$ This form of the transport equation is used with the observation that the equation that is its adjoint is not radically different. In fact, the adjoint equation may be changed to an equation having a form identical to an integro-differential transport equation with a transformation that simply reflects the direction-of-flight coordinates (and reverses the time coordinate for time-dependent problems). The resulting equation not only has the same form but also satisfies the same boundary conditions as the original transport equation. It is therefore straightforward to sample from this transformed adjoint equation. The important consideration is to sample in 
such a way that variances are within acceptable limits for a fixed amount of computational effort.

The integro-differential transport equation also is a convenient starting point for deriving various methods for scoring quantities of interest and for providing relations between estimators.

The integral transport equation and the integro-differential transport equation provide useful tools for improving and understanding Monte Carlo calculations of particle transport. We now turn to a viewpoint that is in some respects less mathematical in nature.

\section{PHYSICAL APPROACH}

Particle transport can be simulated without even referring to the transport equation. This should not be too surprising, since the particle transport itself is a stochastic process and a direct simulation should also be stochastic in nature. In contrast, the transport equation represents an ensemble average of the more-complicated forward Chapman-Kolmogorov equation.

One of the basic advantages of the Monte Carlo method is that the analog Monte Carlo calculation can be carried out by simulating the physics at each point in the history of a particle. This enables us to concentrate the computational effort in a small portion of phase space near the current particle of interest. All that is required for the simulation of the history is a probabilistic description of what may happen to the particle at each point in its history. This generally includes a description of the geometrical boundaries of regions, a description of the material composition within each region, and an adequate description of the cross sections for each isotope. These cross sections also include energy- and angle-transfer frequency functions for all relevant events.

The construction of a Monte Carlo calculation by simulating the Markov process is demonstrated in a book by Cashwell and Everett. ${ }^{10}$ Many of the practical problems associated with Monte Carlo calculations of neutron and photon transport are discussed in this book, but the transport equation is never displayed.

\section{REFERENCES}

1. J. Spanier and E. M. Gelbard, Monte Carlo Principles and Neutron Transport Problems, Addison-Wesley Publishing Company, Reading, Mass., 1969.

2. G. Goertzel and M. H. Kalos, Monte Carlo Methods in Transport Problems, in Progress in Nuclear Energy, Physics and Mathematics, Series I, Vol. 2, pp. 315-369. Pergamon Press, Inc., New York, 1958.

3. R. R. Coveyou, V. R. Cain, and K. J. Yost, Adjoint and Importance in Monte Carlo Applications, Nucl. Sci. Eng., 27: 219-234 (1967). 
4. J. Spanier, Monte Carlo Methods and Their Application to Neutron Transport Problems, USAEC Report WAPD-195, Bettis Atomic Power Laboratory, 1959.

5. D. C. Irving, The Adjoint Boltzmann Equation and Its Simulation by Monte Carlo, USAEC Report ORNL-TM-2870, Oak Ridge National Laboratory, 1970.

6. F. H. Clark, The Exponential Transform as an Importance-Sampling Device-A Review, USAEC Report ORNL-RSIC-14, Oak Ridge National Laboratory, 1966.

7. L. L. Carter, MCNA: A Computer Program To Solve the Adjoint Neutron Transport Equation by Coupled Sampling with the Monte Carlo Method, USAEC Report LA-4488, Los Alamos Scientific Laboratory, 1971.

8. L. B. Levitt and J. Spanier, A New Non-Multigroup Adjoint Monte Carlo Technique, Nucl. Sci. Eng., 37: 278-287 (1969).

9. L. L. Carter and N. J. McCormick, Coupled Sampling with the Monte Carlo Mcthod in Neutron Transport Calculations, Nucl. Sci. Eng., 39: 296-310 (1970).

10. E. D. Cashwell and C. J. Everett, A Practical Manual on the Monte Carlo Method for Random Walk Problems, Pergamon Press, Inc., New York, 1959. 


\section{MECHANICS OF SIMULATING 4 PARTICLE TRANSPORT}

\section{INTRODUCTION}

In this chapter we consider transport processes under the assumption that (1) particles travel in straight lines without losing energy between collision points, (2) particles suffer a collision per unit distance with probability $\Sigma_{t},(3)$ the transition time between entering and leaving a collision can be neglected, and (4) the particle transport being simulated is linear. We assume that satisfactory density functions for modeling the physical processes are available. Special considerations applicable to neutrons and photons are discussed in Chaps. 5 and 6, respectively.

\section{GEOMETRICAL CONSIDERATIONS}

There are advantages to describing particle flight paths by Cartesian coordinates even though the problems of interest may have special symmetries, for example, spherically symmetric systems. A particle's direction of flight in Cartesian coordinates is uniquely specified by the direction cosines $(u, v, w)$ with respect to the $x, y$, and $z$ axes, respectively. The new position of the particle after traveling a distance $\lambda$ is simply

$$
\begin{aligned}
& x^{\prime}=x+u \lambda \\
& y^{\prime}=y+v \lambda \\
& z^{\prime}=z+w \lambda
\end{aligned}
$$

where $(x, y, z)$ was the old position. 
The direction cosines do not change along flight paths, which is a distinct advantage of Cartesian coordinates. However, the direction cosines do change at scattering events. The scattering angle with respect to the incident direction of flight is sampled from an appropriate distribution, which will be discussed later. For isotropic media the final direction on the cone determined by the scattering angle is selected uniformly. A routine for finding the new $\left(u^{\prime}, v^{\prime}, w^{\prime}\right)$, given the precollision $(u, v, w)$, is shown in Fig. 4.1. Here $\mu_{\mathrm{lab}}$ is the cosine of the scattering angle in the laboratory system found before entering this routine. The mathematical relations used in Fig. 4.1 to compute the new $\left(\mathrm{u}^{\prime}, \mathrm{v}^{\prime}, \mathrm{w}^{\prime}\right)$ are derived in Ref. 1 . We also note that the cosine and sine of an angle uniformly distributed from 0 to $2 \pi$ are required for the new $\left(\mathrm{u}^{\prime}, \mathrm{v}^{\prime}, \mathrm{w}^{\prime}\right)$. These functions were obtained by the rejection method described in Chap. 2.

The Monte Carlo computer program is usually designed to solve transport problems consisting of contiguous regions. The material properties within each region are uniform in order to make data storage tractable. In the usual application the boundaries of a region are specified by one or more quadratic surfaces of the general form

$$
\mathrm{Ax}^{2}+\mathrm{By}^{2}+\mathrm{Cz}^{2}+\mathrm{Dxy}+\mathrm{Eyz}+\mathrm{Fzx}+\mathrm{Gx}+\mathrm{Hy}+\mathrm{Jz}+\mathrm{K}=0
$$

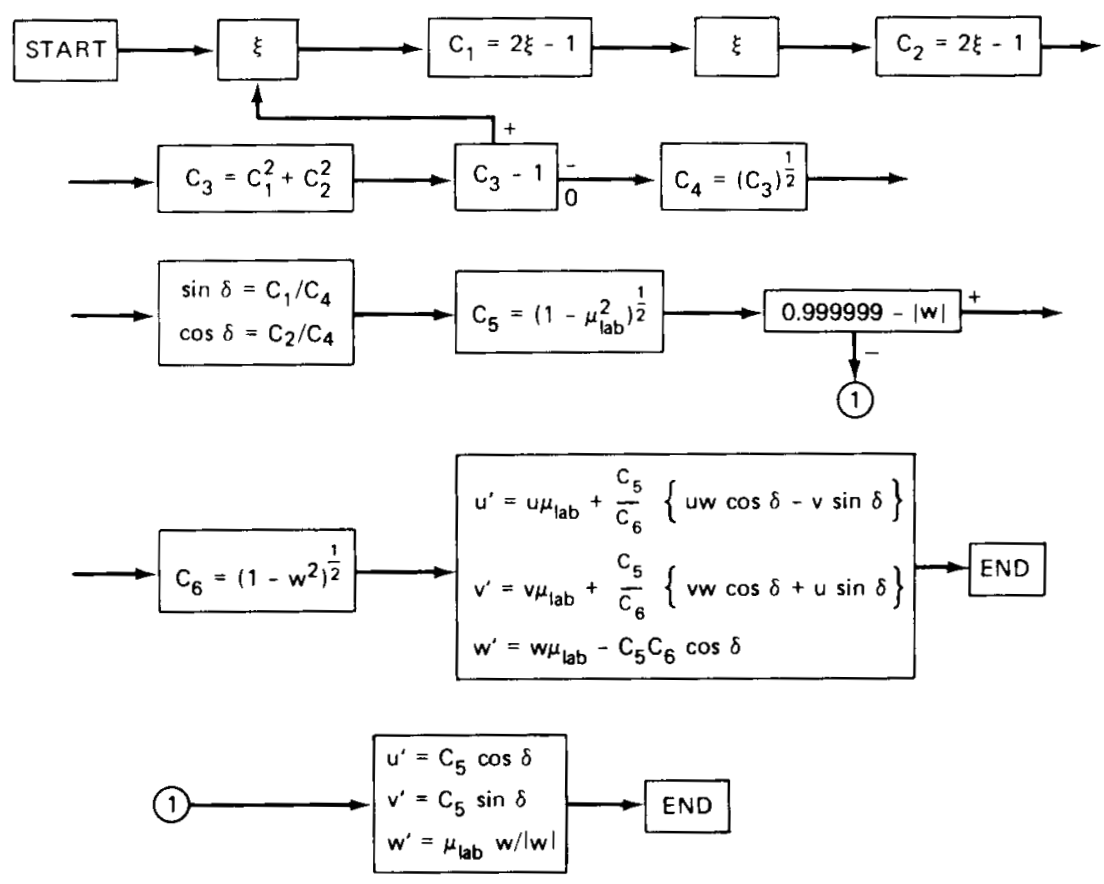

Fig. 4.1 Routine to compute the direction of flight after a scattering collision. 
where $\mathrm{A}, \mathrm{B}, \ldots, \mathrm{K}$ are constants. For a simple surface, such as a plane, sphere, or cylinder, several coefficients are zero, and the computer program is typically written to treat these as special cases of the more general equation in order to save computation time.

A region can be specified by its bounding surfaces and by the sense of the region with respect to each bounding surface. The sense of the region is used to determine on which side of a surface the region lies. The usual convention is that a positive sense means that any $(\mathrm{x}, \mathrm{y}, \mathrm{z})$ point within the region results in a positive value for the lefthand side of Eq. (4.4).

Since it is beyond the scope of this review to examine geometry questions in detail, the interested reader is referred to Refs. 2 to 4 for an introduction to the subject. The so-called combinatorial geometry is discussed in Ref. 3. This technique is intended to simplify the problem specification by allowing the user to model complicated problems from intersections and unions of such simple shapes as spheres, parallelepipeds, cylinders, and truncated cones.

\section{TIME DEPENDENCE}

Time dependence is included in Monte Carlo calculations with a trivial amount of additional effort, which is in contrast to discrete-ordinates calculations. Time dependence is accounted for simply by advancing the time of the particle after each flight and scoring in the appropriate time bins for the functionals of interest.

\section{SAMPLING THE SOURCE}

An extraneous source distribution is specified by an unnormalized density function $\mathrm{S}(\mathrm{r}, \mathrm{E}, \Omega, \mathrm{t})$. The source is relatively easy to bias in order to improve the efficiency of the Monte Carlo calculation, and even a poor guess regarding an importance function can reduce statistical errors. Furthermore, biasing the source distribution is not potentially as dangerous as many other biasing techniques since only the initial particle weight is affected. For generality it will be assumed that a guess regarding the importance function is $\mathrm{I}(\mathrm{r}, \mathrm{E}, \Omega, \mathrm{t})$, although typically an importance function for source biasing will be relatively simple and may, for example, only depend upon energy.

The source coordinates are selected with the density function

$$
g(r, E, \Omega, t)=\frac{S(r, E, \Omega, t) I(r, E, \Omega, t)}{\int S(r, E, \Omega, t) I(r, E, \Omega, t) d^{3} r d E d \Omega d t}
$$

and the initial weight assigned to the source particle is

$$
W=\frac{S(r, E, \Omega, t)}{g(r, E, \Omega, t)}
$$


where the coordinates $(r, E, \Omega, t)$ of the particle were selected from $g$. The function $g$ can be conveniently split into the product of conditional density functions for each variable to simplify the sampling. Examples of sampling from some common source functions are given by Cashwell and Everett. ${ }^{\text {I }}$

\section{ATTENUATION AND LEAKAGE}

The probability that a particle beginning a flight path at $s=0$ will suffer its next collision in some distance $\Delta s$ about $s$ is given by

$$
p(s) \Delta s=\Sigma_{t}(s) \exp \left(-\int_{0}^{s} \Sigma_{t} d s^{\prime}\right) \Delta s
$$

The corresponding cumulative distribution function is

$$
P(s)=1-\exp \left(-\int_{0}^{s} \Sigma_{t} d s^{\prime}\right)
$$

so that

$$
\int_{0}^{s} \Sigma_{t} d s^{\prime}=-\ln [1-P(s)]
$$

For sampling purposes the cumulative distribution function $\mathrm{P}(\mathrm{s})$ is uniformly distributed on the unit interval, and hence $[1-P(s)]$ must also be uniformly distributed on the unit interval; so

$$
\int_{0}^{\mathrm{s}} \Sigma_{\mathrm{t}} \mathrm{ds} \mathrm{s}^{\prime}=-\ln \xi
$$

This equation may be used in the random walk to sample for the distance s to a collision point. For the typical case when the total cross section is sectionally constant across regions, the Markov nature of the particle transport can be used to simplify the sampling. Then, if a random number $\xi$ is greater than $\exp \left(-\Sigma_{t_{1}} s_{1}\right)$, where $s_{1}$ is the distance to the first region boundary, the distance to the collision point is determined as

$$
\mathrm{s}=\frac{-\ln \xi}{\Sigma_{\mathrm{t} 1}}
$$

If the random number $\xi$ is less than $\exp \left(-\Sigma_{11} s_{1}\right)$, the particle reaches the region boundary without suffering a collision. In this case the particle coordinates are advanced to the boundary, and the procedure is repeated for the next region.

In some situations $\Sigma_{\mathrm{t}}$ is not constant across regions. The inverse solution of $\mathrm{Eq}$. (4.10) may then require excessive computational effort since it must be evaluated for each flight path. Alternate sampling schemes are described in Ref. 5 for this special case. 
With some importance sampling methods, the selection of the distance to a collision point is itself altered; so the total cross section appearing in the previous equations is actually a fictitious total cross section. The particle weight at the end of the flight path is adjusted to obtain unbiased results. If $\bar{\Sigma}_{t}(s)$ is the fictitious total cross section used to sample the distance to a collision point, unbiased answers are obtained by multiplying the particle weight, $\mathrm{W}$, by the ratio of the analog to the fictitious density functions evaluated at the collision point $\mathrm{s}$ :

$$
W^{\prime}=\frac{W \Sigma_{t}(s) \exp \left(-\int_{0}^{s} \Sigma_{t} d s^{\prime}\right)}{\left[\bar{\Sigma}_{t}(s) \exp \left(-\int_{0}^{s} \bar{\Sigma}_{t} d s^{\prime}\right)\right]}
$$

Care must be exercised in choosing a proper $\bar{\Sigma}_{t}(\mathrm{~s})$ to prevent undesirable particleweight fluctuations from the frequent adjustments of the particle weight. The wellknown exponential transform has this stability property.

It is often necessary to show that a proposed sampling scheme or estimator is correct in the sense that it leads to unbiased answers. We will illustrate a verification for flight paths sampled with the fictitious total cross section $\bar{\Sigma}_{t}(\mathrm{~s})$. The correctness of the sampling scheme or estimator can be shown by focusing on some infinitesimal section of the flight path $\Delta s^{\prime}$ about $s^{\prime}$ to demonstrate that the correct expected contribution within $\Delta s^{\prime}$ is made from the projected flight of the particle beginning at $s=0$. Let $f(s)$ be the Monte Carlo estimate of the function of interest in $\Delta s^{\prime}$ due to the selected collision point $\mathrm{s}$ in the random walk. Then the expected value of $\mathrm{f}$ for a particle starting at $s=0$ is

$$
E(f)=\int_{0}^{\infty} p(s) f(s) d s
$$

where $p(s)$ is the density function for selecting the collision point $s$ in the random walk. This is illustrated here for two examples.

We show that the weight-correction factor in Eq. (4.12) yields the correct expected number of collisions in $\Delta s^{\prime}$ about $s^{\prime}$ for the random walk itself. In this case, with an initial particle weight of $\mathrm{W}$ at $\mathrm{s}=0$,

$$
\begin{aligned}
& p(s)=\bar{\Sigma}_{t}(s) \exp \left(-\int_{0}^{s} \bar{\Sigma}_{t} d s^{\prime \prime}\right) \\
& \left.f(s)=0 \quad \text { (for s not contained in } \Delta s^{\prime} \text { about } s^{\prime}\right)
\end{aligned}
$$

and

$$
f(s)=\frac{W \Sigma_{t}(s) \exp \left(-\int_{0}^{s} \Sigma_{t} d s^{\prime \prime}\right)}{\left[\bar{\Sigma}_{t}(s) \exp \left(-\int_{0}^{s} \bar{\Sigma}_{t} d s^{\prime \prime}\right)\right]}
$$

for $s$ contained in $\Delta s^{\prime}$ about $s^{\prime}$, i.e., the adjusted particle weight at the collision point. 
Then

$$
E(f)=W \Sigma_{t}\left(s^{\prime}\right) \exp \left(-\int_{0}^{s^{\prime}} \Sigma_{t} d s^{\prime \prime}\right) \Delta s^{\prime}
$$

is the correct expected weight of collisions.

The second example is the verification that a proper path-length estimator to use with the previous sampling scheme is simply the triple product of the new particle weight at the collision point selected in the random walk, the path length across the cell of interest, and the scoring cross section $\Sigma(s)$ of interest. An infinitesimal segment, $\Delta \mathrm{s}^{\prime}$ about $\mathrm{s}^{\prime}$, of the projected flight path is assumed to cross the cell of interest. The particle weight at the collision point is given by Eq. (4.12). The path-length score of the proposed estimator is

$$
f(s)=\frac{W \Sigma\left(s^{\prime}\right) \Sigma_{t}(s) \exp \left(-\int_{0}^{s} \Sigma_{t} d s^{\prime \prime}\right) \Delta s^{\prime}}{\left[\bar{\Sigma}_{t}(s) \exp \left(-\int_{0}^{s} \bar{\Sigma}_{t} d s^{\prime \prime}\right)\right]}
$$

for $s>s^{\prime}$. For $s<s^{\prime}$ the flight path does not cross the cell; thus

$$
f(s)=0 \quad\left(\text { for } s<s^{\prime}\right)
$$

Using the density function for selecting the collision point given by Eq. (4.14),

$$
E(f)=\int_{s^{\prime}}^{\infty} \bar{\Sigma}_{\mathrm{t}}(\mathrm{s}) \exp \left(-\int_{0}^{s} \Sigma_{\mathrm{t}} \mathrm{d} \mathrm{s}^{\prime \prime}\right) \frac{W \Sigma\left(\mathrm{s}^{\prime}\right) \Sigma_{\mathrm{t}}(\mathrm{s}) \Delta \mathrm{s}^{\prime} \exp \left(-\int_{0}^{s} \Sigma_{\mathrm{t}} \mathrm{d} \mathrm{s}^{\prime \prime}\right) \mathrm{ds}}{\bar{\Sigma}_{\mathrm{t}}(\mathrm{s}) \exp \left(-\int_{0}^{s} \bar{\Sigma}_{\mathrm{t}} \mathrm{ds} \mathrm{s}^{\prime \prime}\right)}
$$

which simplifies to the correct analy tic value

$$
E(f)=W \Sigma\left(s^{\prime}\right) \Delta s^{\prime} \exp \left(-\int_{0}^{s^{\prime}} \Sigma_{t} d s^{\prime \prime}\right)
$$

The extension of the above analysis to large $\Delta \mathrm{s}^{\prime}$ and to more complicated situations is obvious. This type of proof should be used whenever there is doubt about the correctness of a proposed sampling scheme. The correct expected value should result for a valid scheme.

\section{SAMPLING THE COLLISION EVENT}

The collision kernel tends to be a complicated and unwieldy function to sample directly. Considerable simplification both conceptually and numerically is obtained 
by expressing the kernel as the sum of contributions from the various events that correspond to the physical model of the possible interactions. It is standard practice to express the kernel as a sum of the contributions from each isotope,

$$
\mathrm{C}\left(\mathrm{E}^{\prime}, \Omega^{\prime} ; \mathrm{E}, \Omega, \mathbf{r}\right)=\sum_{\mathrm{i}} \frac{\Sigma_{\mathrm{t}}(\mathbf{r}, \mathrm{E})_{\mathrm{i}}}{\Sigma_{\mathrm{t}}(\mathrm{r}, \mathrm{E})} \mathrm{C}\left(\mathrm{E}^{\prime}, \Omega^{\prime} ; \mathrm{E}, \Omega, \mathrm{r}\right)_{\mathrm{i}}
$$

where the subscript i denotes the contribution from the $\mathrm{i}$ th isotope and the primes denote postcollision conditions. The $\mathrm{i}$ th isotope is randomly picked to participate in the collision with probability $\Sigma_{\mathrm{t}}(\mathrm{r}, \mathrm{E})_{\mathrm{i}} / \Sigma_{\mathrm{t}}(\mathrm{r}, \mathrm{E})$. The attention in the random walk is then focused on the conditional function $C\left(E^{\prime}, \Omega^{\prime} ; E, \Omega, r\right)_{i}$ for the ith isotope selected rather than on the entire collision kernel.

The collision kernel for the $\mathrm{i}$ th isotope is simplified still further by expressing it as the sum of contributions from various physical events, such as capture, elastic scattering, inelastic scattering, and fission;

$$
\mathrm{C}\left(\mathrm{E}^{\prime}, \Omega^{\prime} ; \mathrm{E}, \Omega, \mathbf{r}\right)_{\mathrm{i}}=\sum_{\mathrm{k}} \frac{\sigma(\mathbf{r}, \mathrm{E})_{\mathrm{ik}}}{\sigma_{\mathrm{t}}(\mathbf{r}, \mathrm{E})_{\mathrm{i}}} \mathrm{C}\left(\mathrm{E}^{\prime}, \Omega^{\prime} ; \mathrm{E}, \Omega, \mathbf{r}\right)_{\mathrm{ik}}
$$

where $\mathrm{k}$ denotes an event. A particular event is then selected to occur in the random walk with a frequency proportional to its cross section $\sigma(\mathrm{r}, \mathrm{E})_{\mathrm{ik}}$. An exception to this procedure for selecting the event is sometimes made for capture.

Capture can be treated in the analog manner just described, where the history is simply terminated after a capture event in the random walk. A more common technique in Monte Carlo computer codes is to reduce the particle weight with the noncapture probability $\left[\sigma_{t}(\mathrm{r}, \mathrm{E})_{\mathrm{i}}-\sigma_{\mathrm{c}}(\mathrm{r}, \mathrm{E})_{\mathrm{i}}\right] / \sigma_{1}(\mathrm{r}, \mathrm{E})_{\mathrm{i}}$ after a collision with the $\mathrm{i} t h$ isotope and then to sample the type of event from the other possible events with probabilities normalized to exclude capture. The advantage of this technique is that the statistical fluctuation due to the particle termination probability is eliminated; however, the disadvantage is that some of the particle weights may eventually become very small so that a significant fraction of machine time is wasted on particles with such small weights that they do not significantly contribute to any estimate. It is also possible for the Monte Carlo population to become supercritical when capture is not allowed to occur; i.e., increase without limit even though the physical system is subcritical. Because of these disadvantages the above treatment is usually augmented with a lower weight cutoff: Whenever the particle weight falls below this cutoff, Russian roulette is played, and the particle history is either terminated or allowed to continue with a larger weight determined such that the expected weight is conserved.

The conditional kernel $\mathrm{C}\left(\mathrm{E}^{\prime}, \Omega^{\prime} ; \mathrm{E}, \Omega, \mathbf{r}\right)_{\mathrm{ik}}$ for the event selected is normalized to unity. The particle weight can either be multiplied by the mean number of secondaries per collision, or, when the mean number is greater than 1, more than one particle can be allowed to emerge from the collision in the Monte Carlo simulation. It is 
usually desirable to break the normalized kernel for a particular noncapture event into the product of a marginal density function for either the emerging energy or direction of flight and a conditional density function for the remaining random variable. The marginal density function is used to select the appropriate random variable, and the conditional density function is used to select the remaining random variable. The physics of the event usually dictates the order for the random variables. For example, elastic scattering is usually treated by selecting the scattering angle, in the center-ofmass system, and then the energy is uniquely determined from the scattering angle. In contrast to this, there are some events, such as fission, where the scattering angle and emerging energy are assumed to be uncorrelated. Then the order of the selection is unimportant. Special cases of interest are discussed more fully in the neutron- and photon-transport chapters.

\section{ESTIMATORS}

The majority of the currently used estimators in Monte Carlo codes are of four basic types. These are the collision estimator, the last-event estimator, the track-length estimator, and the next-event (point-detector) estimator. The use of these four estimators is briefly discussed here for analog sampling. If importance sampling methods are used, these estimators may require alteration to obtain unbiased results.

The functional of interest for discussion purposes is assumed to be the number of reactions in some portion of phase space denoted by $V_{c}$. The macroscopic reaction cross section is denoted by $\Sigma$.

The collision estimator for the functional scores $W \Sigma(r, E) / \Sigma_{t}(r, E)$ at each collision event that occurs within the phase-space volume $V_{c}$. The last-event estimator differs from the collision estimator in that the scoring occurs only when the particle history is terminated by capture. The score at each such capture event, which occurs in the phase-space volume $V_{c}$, is $W \Sigma(r, E) / \Sigma_{c}(r, E)$. This is an unbiased estimator provided the macroscopic capture cross section $\Sigma_{c}(r, E)$ does not vanish at any phasespace point where $\Sigma(\mathrm{r}, \mathrm{E})$ is finite. One might expect the collision estimator to yield a lower variance than the last-event estimator since a score is made at every collision. This is generally verified in practice, although there are converse cases that can occur. For example, the theoretical zero-variance scheme, which uses importance sampling to obtain the correct answer with each sample, invokes a last-event estimator.

Both the collision estimator and last-event estimator tend to suffer statistically in optically thin regions since few collisions occur there. This statistical problem is usually improved with the use of a path-length estimator. The path-length estimator scores $\left[\mathrm{W} \int_{0}^{\mathrm{s}} \Sigma(\mathrm{r}, \mathrm{E}) \mathrm{ds} \mathrm{s}^{\prime}\right]$ for each track length in the phase-space volume $\mathrm{V}_{\mathrm{c}}$, where $\mathrm{s}$ is the path length within $V_{c}$ of a particle with weight $W$. The path-length estimator enjoys extensive use since it has excellent properties in optically thin regions and tends to perform satisfactorily for optically thick regions as well. Spanier and Gelbard ${ }^{6}$ show that the track-length estimator is a limiting form of the collision estimator. 
This is obtained by adding a fictitious "straight-ahead" scattering contribution to the usual scattering term and increasing the attenuation term to obtain a balance.

The variance of the path-length and collision estimators can be computed analytically for the simple case of a beam of monoenergetic particles incident normally on a slab of material with $\Sigma_{c}=\Sigma_{t}$. The resulting expressions for the relative errors (ratio of the square root of the variance to the mean) in the collision probability per incident particle for a slab that is $\mathrm{x}$ mean free paths thick are

$$
\begin{aligned}
{\left[\begin{array}{l}
\text { Relative error of } \\
\text { collision estimator }
\end{array}\right] } & =\left[\frac{\mathrm{e}^{-\mathrm{x}}}{\left(1-\mathrm{e}^{-\mathrm{x}}\right)}\right]^{1 / 2} \\
& \rightarrow \mathrm{x}^{-1 / 2} \text { as } \mathrm{x} \rightarrow 0 \\
& \rightarrow 0 \quad \text { as } \mathrm{x} \rightarrow \infty \\
{\left[\begin{array}{l}
\text { Relative error of } \\
\text { path-length estimator }
\end{array}\right] } & =\frac{\left(1-2 \mathrm{xe}^{-\mathrm{x}}-\mathrm{e}^{-2 \mathrm{x}}\right)^{1 / 2}}{\left(1-\mathrm{e}^{-\mathrm{x}}\right)} \\
& \rightarrow(\mathrm{x} / 3)^{1 / 2} \text { as } \mathrm{x} \rightarrow 0 \\
& \rightarrow 1 \quad \text { as } \mathrm{x} \rightarrow \infty
\end{aligned}
$$

The relative errors are shown in Fig. 4.2 as a function of $x$. The path-length estimator is clearly preferable for thin shells, but the collision estimator is better for thick shells with the crossover point at $x=1.256$. One must remember, however, that this is a special case, and the extension of these results to more complicated situations must be made with caution.

The collision, last-event, and track-length estimators all tend to suffer from increasing statistical errors as the volume of the detector region becomes arbitrarily small. The next-event (point-detector) estimator is a candidate for such problems. This estimator may be understood by beginning with the integral transport equation

$$
F\left(R^{\prime}\right)=\int F(R) K\left(R^{\prime} ; R\right) d R+S\left(R^{\prime}\right)
$$

where $\mathrm{R}^{\prime}$ denotes the detector phase-space point $\left(\mathbf{r}^{\prime}, \mathrm{E}^{\prime}, \Omega^{\prime}\right)$. The detector point $\mathbf{r}^{\prime}$ and the collision point $\mathbf{r}$ fix the emerging direction of flight necessary to reach the detector after the collision; so it is convenient to express $K\left(R^{\prime} ; R\right)$ as 


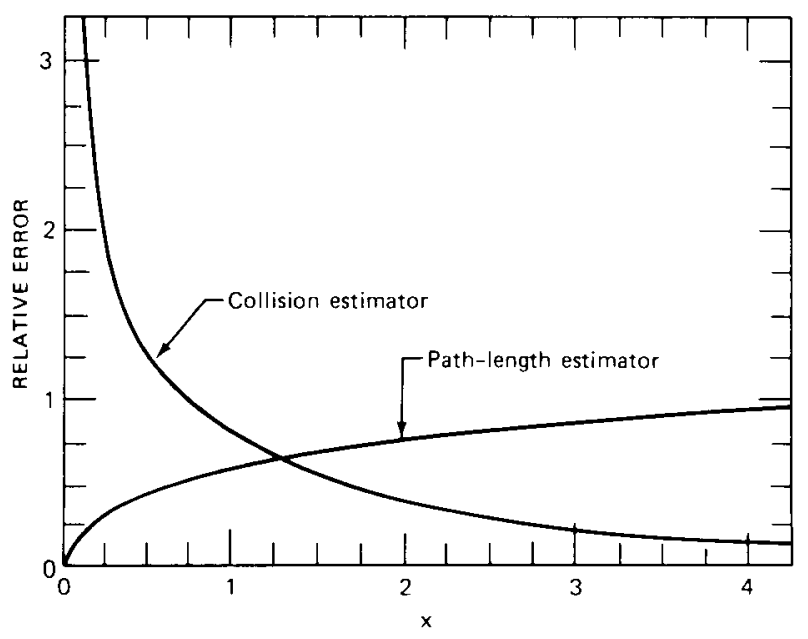

Fig. 4.2 Relative errors of path-length estimator and collision estimator for a slab that is $\mathrm{x}$ mean free paths thick.

$$
\begin{aligned}
\mathrm{K}\left(\mathrm{R}^{\prime} ; \mathrm{R}\right)= & \sum_{\mathrm{i}} \frac{\Sigma_{\mathrm{t}}(\mathbf{r}, \mathrm{E})_{\mathrm{i}}}{\Sigma_{\mathrm{t}}(\mathbf{r}, \mathrm{E})} \sum_{\mathrm{k}}\left\{\frac{\sigma(\mathbf{r}, \mathrm{E})_{\mathrm{ik}}}{\sigma_{\mathrm{t}}(\mathrm{r}, \mathrm{E})_{\mathrm{i}}} \mathrm{C}\left(\mathrm{E}^{\prime}, \Omega^{\prime} ; \mathrm{E}, \Omega, \mathrm{r}\right)_{\mathrm{ik}}\right. \\
& \left.\times \delta\left[\Omega^{\prime}-\frac{\left(\mathbf{r}^{\prime}-\mathbf{r}\right)}{\left|\mathbf{r}-\mathbf{r}^{\prime}\right|}\right] \frac{\exp \left[-\int_{0}^{\left|\mathbf{r}^{\prime}-\mathbf{r}\right|} \Sigma_{\mathrm{t}}\left(\mathbf{r}+\Omega^{\prime} \mathrm{s}^{\prime}, \mathrm{E}^{\prime}\right) \mathrm{ds^{ \prime } ]}\right.}{\left|\mathbf{r}-\mathbf{r}^{\prime}\right|^{2}} \Sigma_{\mathrm{t}}\left(\mathbf{r}^{\prime}, \mathrm{E}^{\prime}\right)\right\}
\end{aligned}
$$

where the notation is that used in the section on sampling the collision event. The random walk calculates the integral over $\mathrm{R}$ by simply scoring the product of the particle weight at each collision with the kernel $\mathrm{K}$ of Eq. (4.27). Typically the detector score of interest is

$$
F\left(R^{\prime}\right) \frac{\Sigma\left(r^{\prime}, E^{\prime}\right)}{\Sigma_{t}\left(r^{\prime}, E^{\prime}\right)} d \Omega^{\prime} d E^{\prime}
$$

integrated over $4 \pi$ and some energy bin; so the delta function in Eq. (4.27) and a possible delta function in the kernel $\mathrm{C}$ are removed. Here $\Sigma$ is the cross section of the reaction being considered at the point detector ( $\Sigma$ is set to unity for a flux calculation).

The integral of the kernel $\mathrm{K}$ over an energy bin is of ten impractical to evaluate at each collision in the Monte Carlo simulation. Hence the isotope $\mathrm{i}$ and event $\mathrm{k}$ are 
usually sampled in the random walk before scoring the point detector. If elastic scattering with a target nucleus at rest is the event selected, the required angle to scatter to the detector uniquely determines the final energy; so the score for this event is

$$
\frac{\mathrm{Wh}\left(\mu_{\mathrm{lab}} ; E\right)_{\text {lab }}}{2 \pi\left|\mathbf{r}-\mathbf{r}^{\prime}\right|^{2}} \Sigma\left(\mathbf{R}^{\prime}\right) \exp \left[-\int_{0}^{\left|\mathbf{r}^{\prime}-\mathbf{r}^{\prime}\right|} \Sigma_{\mathrm{t}} \mathrm{ds} \mathbf{s}^{\prime}\right]
$$

whenever the final energy lies within the energy bin of interest. Here $\mu_{\text {lab }}$ is the cosine of the scattering angle in the laboratory system,

$$
\mu_{\mathrm{lab}}=\Omega \cdot \Omega^{\prime}
$$

and $h\left(\mu_{\mathrm{lab}} ; \mathrm{E}\right)_{\mathrm{lab}}$ is the density function for the particle to scatter elastically through $\mu_{\mathrm{lab}}$ with $\Omega^{\prime}$ determined by the detector position. If $h$ is given in the center-of-mass system, the appropriate transformation must be made. The pertinent relation for the elastic scattering of a neutron is

$$
\begin{aligned}
\mathrm{h}\left(\mu_{\mathrm{lab}} ; \mathrm{E}\right)_{\mathrm{lab}}= & \frac{\mathrm{h}\left(\mu_{\mathrm{cm}} ; \mathrm{E}\right)_{\mathrm{cm}}}{\mathrm{A}}\left[2 \mu_{\mathrm{lab}}+\left(\mathrm{A}^{2}+\mu_{\mathrm{lab}}^{2}-1\right)^{1 / 2}\right. \\
& \left.+\frac{\mu_{\mathrm{lab}}^{2}}{\left(\mathrm{~A}^{2}+\mu_{\mathrm{lab}}^{2}-1\right)^{1 / 2}}\right]
\end{aligned}
$$

where

$$
\mu_{\mathrm{cm}}=\frac{1}{\mathrm{~A}}\left[\mu_{\mathrm{lab}}^{2}-1+\mu_{\mathrm{lab}}\left(\mathrm{A}^{2}+\mu_{\mathrm{lab}}^{2}-1\right)^{1 / 2}\right]
$$

The final energy and scattering angle have a one-to-one positive correlation for elastic scattering, i.e., knowledge of one uniquely determines the other. In contrast to this, some events are modeled so that the emerging energy and the scattering angle in the laboratory system are uncorrelated. The appropriate point-detector score for such an event is

$$
\frac{W h\left(\mu_{\mathrm{lab}} ; \mathrm{E}\right)_{\mathrm{ik}, \mathrm{lab}}}{2 \pi\left|\mathbf{r}-\mathbf{r}^{\prime}\right|^{2}} \nu(\mathrm{E}) \Sigma\left(\mathrm{R}^{\prime}\right) \exp \left(-\int_{0}^{\left|\mathbf{r}^{\prime}-\mathrm{r}\right|} \dot{\Sigma}_{\mathrm{t}} \mathrm{ds^{ \prime }}\right)
$$

whenever the final energy lies within the energy bin of interest. Here $\mathrm{h}$ is the density function in the laboratory system for scattering through $\mu_{\mathrm{lab}}, \nu$ is the number of secondaries for the event, and the final energy $E^{\prime}$ is sampled from the density function for the kinetic energy of a particle emerging from the event. 
A variety of physical models are used which lie between the completely correlated elastic-scattering case and the uncorrelated event just considered. We will not discuss any of these additional possibilities here, although the appropriate pointdetector score for the inelastic scattering of a neutron is derived in the section on inelastic scattering in Chap. 5, and the point-detector score for the thermal scattering of neutrons using a free gas model is given in the section on thermal scattering in Chap. 5. It should also be noted that the first-flight contribution from the extraneous source to the point detector must be scored for each source particle. This first-flight contribution can often be computed analytically. For more complex sources the Monte Carlo method can be used to compute the necessary integrals.

The next-event estimator suffers from two very severe limitations that become apparent in practical applications. This estimator tends to require a great deal of computational effort since the attenuation distance from each collision to the point detector is required. This becomes a particularly important consideration for geometrically complicated systems or when many point detectors are dictated by a problem. The second limitation of the point detector involves variances. When the detector point lies within a scattering medium, the theoretical second moment of the estimate may be infinite even though the first moment is finite. This is due to the $1 /\left|\mathbf{r}^{\prime}-\mathbf{r}\right|^{2}$ factor in the scoring equation.

Kalos $^{7}$ proposed a once-more-collided estimator to remedy this infinite variance problem. The contribution at the detector point $\mathbf{r}^{\prime}$ is computed for a collision at $\mathbf{r}$ by sampling an intermediate collision point. This method yields a finite second moment with a properly chosen density function for selecting the intermediate collision point. However, it does suffer from the disadvantage that the selection of the intermediate collision point requires additional computer programming effort and increases the running time of the problem. Steinberg and Kalos ${ }^{8}$ later proposed a method to bias the selection of the collision points in the random walk toward the point detector. This also results in a finite second moment, but the particle weights must be corrected to produce unbiased estimates, and it is possible for this resulting weight fluctuation to adversely affect estimates in other portions of phase space.

These two methods of obtaining a finite variance are unbiased. Other methods have been proposed which involve some approximation but, nevertheless, are useful in problems of practical interest. One useful technique is to draw an imaginary sphere of radius $\mathrm{R}$ around the detector point. Any collisions that occur outside this sphere are scored with the usual point-detector equations developed previously. For collisions within the sphere, the factor

$$
\frac{\exp \left(-\int_{0}^{\left|\mathbf{r}-\mathbf{r}^{\prime}\right|} \Sigma_{\mathrm{t}} \mathrm{ds^{ \prime }}\right)}{\left|\mathbf{r}-\mathbf{r}^{\prime}\right|^{2}}
$$

is replaced by its volume average, assuming uniform collisions within the sphere, as

$$
\frac{\int_{0}^{R} e^{-\Sigma_{t}{ }^{r}} d r}{\int_{0}^{R} r^{2} d r}=\frac{1-e^{-\Sigma_{t} R}}{\frac{1}{3}\left(R^{3} \Sigma_{t}\right)}
$$


The variance in the estimator tends to decrease as the imaginary sphere radius $\mathrm{R}$ is increased. On the other hand, the approximation involved by volume averaging tends to be worse for large $\mathrm{R}$. These considerations generally mean that $\mathrm{R}$ is chosen to be some fraction of a mean free path, perhaps in the range $1 / 8$ to $1 / 2$ of a mean free path.

Numerous journal articles (in addition to those just mentioned on the point detector) are devoted almost exclusively to estimators. MacMillan ${ }^{9}$ considers linear combinations of various estimators to minimize the variance. Gelbard, Ondis, and Spanier ${ }^{10}$ and Spanier ${ }^{1} 1$ develop the mathematics for constructing a variety of estimators and discuss some pertinent examples.

\section{SIMULATION OF ADJOINT EQUATIONS}

There would be little practical value in simulating equations that are adjoint to transport equations if all particle-transport problems could be solved by a simple analog calculation. The literature attests to the fact that analog simulations require excessive computational effort for some problems, thus the interest in adjoint calculations both to bias forward simulations as well as to provide an entirely alternate method of solution.

A calculation using an adjoint equation is particularly attractive for two general classes of problems where the solution of the transport equation is difficult if not impossible to obtain with a reasonable amount of computational effort. The first class of problems has the common feature that the phase-space volume of interest for computing some response is small enough that an analog Monte Carlo calculation is inefficient since the particles will seldom pass through the volume of interest. Although the analog Monte Carlo simulation can be improved by biasing techniques or by using a point-detector estimator, there are still many problems that are not amenable to such a direct attack. The second class of problems has the common feature that some response is required as a function of various source distributions. This requires a separate simulation of the transport equation for each source distribution and hence requires considerable computational effort. We will show that a simulation of an adjoint equation has attractive advantages for both these classes of problems. However, before we proceed, we should state that an adjoint-equation approach is not a panacea for all problems that are difficult to solve in a direct way. For example, deep-penetration problems are difficult to solve with acceptable amounts of computational time, but these problems also tend to be difficult to solve with an adjoint simulation since the particle histories must still be tracked through many mean free paths. The difference is that in the simulation of the transport equation particle histories are tracked from the source to the detector, whereas in a simulation of an adjoint equation, particle histories are tracked from the detector back to the source.

Various methods for simulating adjoint equations are reported in the literature. ${ }^{12-17}$ Some investigators consider the simulation of the adjoint to the integral transport equation, and others consider the simulation of the adjoint to the integrodifferential transport equation. We will consider the integro-differential viewpoint 
here for the specific case of neutrons. There are no important differences for gamma rays.

The relevant equations can be derived by beginning with the Boltzmann integrodifferential neutron transport equation for a point source of monoenergetic and monodirectional neutrons,

$$
\begin{aligned}
L G\left(R ; R^{\prime}\right)= & \frac{1}{v} \frac{\partial G\left(R ; R^{\prime}\right)}{\partial t}+\Omega \cdot \nabla G\left(R ; R^{\prime}\right)+\Sigma_{t}(R) G\left(R ; R^{\prime}\right) \\
& -\int_{\Omega^{\prime \prime}} \int_{E^{\prime \prime}} G\left(r, E^{\prime \prime}, \Omega^{\prime \prime}, t ; R^{\prime}\right) \Sigma_{t}\left(\mathbf{r}, E^{\prime \prime}\right) C\left(E, \Omega ; E^{\prime \prime}, \Omega^{\prime \prime}, \mathbf{r}\right) d E^{\prime \prime} d \Omega^{\prime \prime} \\
= & \delta\left(\mathbf{r}-\mathbf{r}^{\prime}\right) \delta\left(E-E^{\prime}\right) \delta\left(\Omega-\Omega^{\prime}\right) \delta\left(t-t^{\prime}\right)
\end{aligned}
$$

where $\mathrm{L}$ is an operator defined by Eq. (4.34). The quantity $\mathrm{R}$ is a shorthand notation denoting the neutron space position $\mathbf{r}$, its kinetic energy $E$, and direction of motion $\Omega$ at time $\mathrm{t}$, and $\mathrm{C}\left(\mathrm{E}, \Omega ; \mathrm{E}^{\prime \prime}, \Omega^{\prime \prime}, \mathrm{r}\right)$ is the collision kernel defined previously. The total cross section $\Sigma_{\mathrm{t}}$ is assumed to be independent of time and direction and thus the notations $\Sigma_{t}(R)$ and $\Sigma_{t}(r, E)$ are used interchangeably. The coordinates to the left of the semicolon in the Green's function $G$ of Eq. (4.34) represent field (i.e., final state) points, and those to the right of the semicolon represent source points. Hence $G\left(R ; R^{\prime}\right)$ is the neutron flux at $R$ due to a unit point source at $R^{\prime}$.

The neutron flux $\phi(R)$ can be expressed in terms of the solution of Eq. (4.34) for the Green's function as

$$
\phi(R)=\int G\left(R ; R^{\prime}\right) S\left(R^{\prime}\right) d R^{\prime}
$$

where $S\left(R^{\prime}\right)$ is the extraneous source density. However, rather than using the Monte Carlo method to compute a point value of the neutron flux, it is more often used to estimate a functional $\mathrm{J}$ (or a number of such functionals) defined as

$$
\begin{aligned}
J & =\int \phi(R) \Sigma(R) d R \\
& =\iint G\left(R ; R^{\prime}\right) S\left(R^{\prime}\right) \Sigma(R) d R^{\prime} d R
\end{aligned}
$$

Here $\Sigma(R)$ is an arbitrary cross section, and the last relation of Eq. (4.36) was obtained from the expression for the neutron flux given by Eq. (4.35). The functional $J$ is computed with Monte Carlo by selecting the $\mathrm{i}$ th source neutron coordinates $\mathrm{R}_{\mathrm{i}}$ with the density function $S\left(R^{\prime}\right)$ and following the history of the neutron (and progeny) to compute the estimate

$$
\mathbf{J}_{\mathrm{i}}=\int \mathrm{G}\left(\mathrm{R} ; \mathbf{R}_{\mathrm{i}}\right) \Sigma(\mathrm{R}) \mathrm{dR} W\left(\mathrm{R}_{\mathrm{i}}\right)
$$

where $W\left(R_{i}\right)$ is the initial weight assigned to the neutron given by $\int S\left(R^{\prime}\right) d R^{\prime}$. The 
functional $\mathbf{J}$ is estimated by sampling from the transport equation, but we will show that a corresponding estimate of $\mathrm{J}$ can be obtained by sampling from the adjoint equation.

The equation for the adjoint Green's function $\mathrm{G}^{+}$is derived by finding an operator $\mathrm{L}^{+}$that satisfies the equation

$$
\int\left[\mathrm{L}^{+} \mathrm{G}^{+}\left(\mathrm{R} ; \mathrm{R}^{\prime \prime}\right)\right] \mathrm{G}\left(\mathrm{R} ; \mathrm{R}^{\prime}\right) \mathrm{dR}=\int \mathrm{G}^{+}\left(\mathrm{R} ; \mathrm{R}^{\prime \prime}\right)\left[\mathrm{LG}\left(\mathrm{R} ; \mathrm{R}^{\prime}\right)\right] \mathrm{dR}
$$

The boundary conditions on $G^{+}\left(R ; R^{\prime \prime}\right)$ are chosen to be consistent with those on $G\left(R ; R^{\prime}\right)$ and are such that the bilinear concomitant is zero, i.e., $G$ is equal to zero for direction-of-flight vectors into the system and $\mathrm{G}^{+}$is equal to zero for direction-offlight vectors out of the system. The definition of the operator $\mathrm{L}^{+}$in Eq. (4.38) with the associated boundary conditions is used to obtain the adjoint equation for the adjoint Green's function as

$$
\begin{aligned}
\mathrm{L}^{+} \mathrm{G}^{+}\left(\mathrm{R} ; \mathrm{R}^{\prime \prime}\right)= & -\frac{1}{\mathrm{v}} \frac{\partial \mathrm{G}^{+}\left(\mathrm{R} ; \mathrm{R}^{\prime \prime}\right)}{\partial \mathrm{t}}-\Omega \cdot \nabla \mathrm{G}^{+}\left(\mathrm{R} ; \mathrm{R}^{\prime \prime}\right)+\Sigma_{\mathrm{t}}(\mathrm{R}) \mathrm{G}^{+}\left(\mathrm{R} ; \mathrm{R}^{\prime \prime}\right) \\
& -\int_{\Omega^{\prime}} \int_{\mathrm{E}^{\prime}} \mathrm{G}^{+}\left(\mathrm{r}, \mathrm{E}^{\prime}, \Omega^{\prime}, \mathrm{t} ; \mathrm{R}^{\prime \prime}\right) \Sigma_{\mathrm{t}}\left(\mathrm{r}, \mathrm{E}^{\prime}\right) \\
& \times\left[\frac{\mathrm{C}\left(\mathrm{E}^{\prime}, \Omega^{\prime} ; \mathrm{E}, \Omega, \mathrm{r}\right) \Sigma_{\mathrm{t}}(\mathrm{r}, \mathrm{E})}{\Sigma_{\mathrm{t}}\left(\mathrm{r}, \mathrm{E}^{\prime}\right)}\right] \mathrm{d} \mathrm{E}^{\prime} \mathrm{d} \Omega^{\prime} \\
= & \delta\left(\mathrm{r}-\mathrm{r}^{\prime \prime}\right) \delta\left(\mathrm{E}-\mathrm{E}^{\prime \prime}\right) \delta\left(\Omega-\Omega^{\prime \prime}\right) \delta\left(\mathrm{t}-\mathrm{t}^{\prime \prime}\right)
\end{aligned}
$$

A reciprocity relation between $\mathrm{G}^{+}$and $\mathrm{G}$ can be derived by multiplying Eq. (4.39) by $G\left(R ; R^{\prime}\right) d R$, multiplying Eq. (4.34) by $G^{+}\left(R ; R^{\prime \prime}\right) d R$, extracting the difference of the resulting two equations, and integrating this difference over all phase space. The subsequent change of variables by replacing $R^{\prime \prime}$ by $R$ yields the familiar form of the reciprocity theorem,

$$
\mathrm{G}^{+}\left(\mathrm{R}^{\prime} ; \mathrm{R}\right)=\mathrm{G}\left(\mathrm{R} ; \mathrm{R}^{\prime}\right)
$$

The reciprocity relation of Eq. (4.40) can be inserted into Eq. (4.36) to obtain an alternate expression for the functional $\mathrm{J}$ as

$$
J=\iint G^{+}\left(R^{\prime} ; R\right) S\left(R^{\prime}\right) \Sigma(R) d R^{\prime} d R
$$

The functional $\mathbf{J}$ of Eq. (4.41) is computed with Monte Carlo by selecting the $\mathrm{i}$ th source pseudoneutron ${ }^{*}$ coordinates $\mathrm{R}_{\mathrm{i}}$ with the density function

*Pseudoneutrons are defined here as those "particles" whose transport is described by the adjoint equation. 


$$
\frac{\Sigma(\mathrm{R})}{\int \Sigma\left(\mathrm{R}^{\prime}\right) \mathrm{d} \mathrm{R}^{\prime}}
$$

and then following the history of pseudoneutrons (and progeny) to compute the estimate

$$
J_{i}=\int G^{+}\left(R^{\prime} ; R_{i}\right) S\left(R^{\prime}\right) d R^{\prime} W\left(R_{i}\right)
$$

where $W\left(R_{i}\right)$ is the initial weight assigned to the pseudoneutron as given by $\int \Sigma\left(R^{\prime}\right) d R^{\prime}$. The roles of the neutron source $\mathrm{S}$ and the scoring cross section $\Sigma$ have been interchanged in this estimation of $\mathrm{J}$ by sampling from the adjoint equation. Thus $\Sigma$ now assumes the role of a source, and $\mathrm{S}$ assumes the role of a scoring cross section. This fact has two well-known implications about the computational effort required for a problem:

1. If a response of some part of the system as a function of the neutron-source distribution is required, it may be more efficient to estimate the functionals by sampling from the adjoint equation. This is because only one adjoint calculation is required rather than a number of separate transport calculations.

2 . If the phase-space volume containing nonzero $\Sigma$ is small, it may be more efficient to estimate $\mathbf{J}$ by sampling from the adjoint equation because all pseudoneutron histories begin in the small phase-space volume.

If the region in phase space where the source does not vanish is not too small and if an efficient scheme for sampling from the adjoint equation is available, then use of the adjoint equation is advantageous.

The approach used here to develop a scheme for sampling from the adjoint equation is to begin by finding a simple way of transforming Eq. (4.39) into an equation identical in form to the transport equation, Eq. (4.34). Techniques for sampling from the resulting equation are therefore well known.

This simple transformation is obtained with the definitions

$$
\begin{aligned}
t_{\mathrm{a}} & =t_{\mathrm{m}}-\mathrm{t} \\
\Omega_{\mathrm{a}} & =-\Omega \\
\mathrm{G}_{\mathrm{a}}^{+}\left(\mathrm{r}, \mathrm{E}, \Omega_{\mathrm{a}}, \mathrm{t}_{\mathrm{a}} ; \mathrm{r}^{\prime \prime}, \mathrm{E}^{\prime \prime}, \Omega_{\mathrm{a}}^{\prime \prime}, \mathrm{t}_{\mathrm{a}}^{\prime \prime}\right) & =\mathrm{G}^{+}\left(\mathrm{r}, \mathrm{E},-\Omega_{\mathrm{a}}, \mathrm{t}_{\mathrm{m}}-\mathrm{t}_{\mathrm{a}} ; \mathbf{r}^{\prime \prime}, \mathrm{E}^{\prime \prime},-\Omega_{\mathrm{a}}^{\prime \prime}, \mathrm{t}_{\mathrm{m}}-\mathrm{t}_{\mathrm{a}}^{\prime \prime}\right)
\end{aligned}
$$

where $t_{m}$ is a maximum time of interest in the problem, $t_{a}$ is the adjoint time, and $\Omega_{a}$ is the direction of motion of the pseudoneutron. Substituting these definitions into Eq. (4.39) yields the equation 


$$
\begin{aligned}
& \frac{1}{\mathrm{v}} \frac{\partial \mathrm{G}_{\mathrm{a}}^{+}\left(\mathrm{R}_{\mathrm{a}} ; \mathrm{R}_{\mathrm{a}}^{\prime \prime}\right)}{\partial \mathrm{t}_{\mathrm{a}}}+\Omega_{\mathrm{a}} \cdot \nabla \mathrm{G}_{\mathrm{a}}\left(\mathrm{R}_{\mathrm{a}} ; \mathrm{R}_{\mathrm{a}}^{\prime \prime}\right)+\Sigma_{\mathrm{t}}\left(\mathrm{R}_{\mathrm{a}}\right) \mathrm{G}_{\mathrm{a}}^{+}\left(\mathrm{R}_{\mathrm{a}} ; \mathrm{R}_{\mathrm{a}}^{\prime \prime}\right) \\
& -\int_{\Omega_{\mathrm{a}}^{\prime}} \int_{\mathrm{E}^{\prime}} \mathrm{G}_{\mathrm{a}}^{+}\left(\mathrm{r}, \mathrm{E}^{\prime}, \Omega_{\mathrm{a}}^{\prime}, \mathrm{t}_{\mathrm{a}} ; \mathrm{R}_{\mathrm{a}}^{\prime \prime}\right) \Sigma_{\mathrm{t}}\left(\mathrm{r}, \mathrm{E}^{\prime}\right)\left[\frac{\mathrm{C}\left(\mathrm{E}^{\prime},-\Omega_{\mathrm{a}}^{\prime} ; \mathrm{E},-\Omega_{\mathrm{a}}, \mathrm{r}\right) \Sigma_{\mathrm{t}}(\mathrm{r}, \mathrm{E})}{\Sigma_{\mathrm{t}}\left(\mathrm{r}, \mathrm{E}^{\prime}\right)}\right] \mathrm{d} \mathrm{E}^{\prime} \mathrm{d} \Omega_{\mathrm{a}}^{\prime} \\
& =\delta\left(\mathrm{r}-\mathrm{r}^{\prime \prime}\right) \delta\left(\mathrm{E}-\mathrm{E}^{\prime \prime}\right) \delta\left(-\Omega_{\mathrm{a}}+\Omega_{\mathrm{a}}^{\prime \prime}\right) \delta\left(-\mathrm{t}_{\mathrm{a}}+\mathrm{t}_{\mathrm{a}}^{\prime \prime}\right)
\end{aligned}
$$

where $R_{a}$ is defined to be the phase-space point $r, E, \Omega_{a}, t_{a}$. Equation (4.45) is identical in form to the neutron transport equation, where the term in brackets in the integrand is recognized as the transfer kernel.

It may also be proved that $\mathrm{G}_{\mathrm{i}}^{+}$satisfies the same boundary conditions as $\mathrm{G}$. The condition on $\mathrm{G}$ is that $\mathrm{G}\left(\mathbf{r}_{s}, \mathrm{E}, \Omega^{-}, t ; \mathbf{R}^{\prime}\right)=0$ at every point $\mathbf{r}_{s}$ on the outer surface of the system, where $\Omega^{-}$denotes any direction into the system. This boundary condition, combined with the requirement that the bilinear concomitant be zero on the outer surface, leads to the following condition for $\mathrm{G}^{+}$:

$$
\mathrm{G}^{+}\left(\mathrm{r}_{\mathrm{s}}, \mathrm{E}, \Omega^{+}, \mathrm{t} ; \mathrm{R}^{\prime \prime}\right)=0
$$

where $\Omega^{+}$denotes any direction out of the system. From Eq. (4.46) and the definition of $\mathrm{G}_{\mathrm{a}}^{+}$, the boundary condition on $\mathrm{G}_{\mathrm{i}}^{+}$demands that no pseudoneutrons enter the system from the outer surface. The time constraint on $G$ is that $G\left(R ; R^{\prime}\right)=0$ for $t^{\prime}>t$. This requirement coupled with the reciprocity theorem of Eq. (4.40) and the definitions in Eq. (4.44) dictate that $G_{a}^{+}\left(R_{a} ; R_{a}^{\prime \prime}\right)=0$ for $t_{a}^{\prime \prime}>t_{a}$.

Since Eq. (4.45) has the same form as the transport equation and also satisfies the same boundary conditions, it would seem to offer no difficulties for a Monte Carlo simulation. However, this is not necessarily the case. The reason for this is an old problem with Monte Carlo calculations. Not only must one develop an unbiased sampling scheme, but from a practical viewpoint this sampling scheme must have a small enough variance so that statistically acceptable answers can be obtained in a reasonable amount of computational effort. To better understand the typical problems that arise with a straightforward sampling of the adjoint equation, we will first make some comments on random-walk simulations of the transport equation.

A large portion of the literature on Monte Carlo has been oriented toward the proper biasing of sampling schemes to reduce variances. However, it should be emphasized that many of the particle-transport problems of current interest are solved with a direct analog simulation or with only small alterations of an analog simulation. Why is the analog random walk acceptable for this large class of problems? The answer tends to be twofold. First, the analog random-walk models the physical process. Hence it tends to be efficient for estimating the class of functionals such that a significant fraction of the source particles contributes to the estimate of the func- 
tional. For this reason analog Monte Carlo tends to work well for many nuclear reactor problems but works poorly for deep-penetration problems. The second reason that analog Monte Carlo works so well in many transport problems involves particle weights. For many problems of interest, the normalization of the collision kernel is unity or less than unity in most of the phase space. That is, the system is not multiplying, is only weakly multiplying, or only the first generation of neutrons from an iterated fission-source distribution is being tracked. This has the practical advantage that the particle population does not multiply drastically (or that particle weights do not increase drastically), which can adversely effect variances.

These two advantages are not usually present in an attempt to do an analog simulation of the transformed adjoint equation, Eq. (4.45). The pseudoneutrons do not necessarily migrate toward regions of interest, and the normalization of the collision kernel appearing in Eq. (4.45) is of ten much greater than unity. This latter effect tends to result in increasing and fluctuating particle weights at collisions or, alternatively, in a multiplying system if some type of weight splitting is used.

It is possible to overcome the first problem in most cases by simply using splitting and Russian roulette to bias the pseudoparticle history toward regions of interest. The weight-fluctuation problem is not so easily resolved. A number of different techniques have been suggested in the literature to alleviate the problem. With all due respect to the proponents of the various techniques, these techniques all seem to suffer from rather severe deficiencies for general applications. Spanier and Habetler 17 used the infinite-medium multigroup thermal-neutron flux to bias the adjoint scattering kernel for an adjoint calculation of thermal-neutron transport. They observed that this technique reduced variances considerably over those obtained in calculations without such biasing. Levitt and Spanier ${ }^{13}$ used a transformation of the adjoint integro-differential equation such that changes in particle weights would only occur at each boundary crossing. They attempted to optimize the adjoint-scattering kernel by using the corresponding infinite-medium flux for each individual region in the problem as a biasing function for that region. One disadvantage of this technique is the infinitemedium flux of a homogeneous region may be quite different from the actual flux in that region in the heterogeneous problem. However, the usefulness of the method was demonstrated in a number of resonance-escape problems. Eriksson et al. ${ }^{15}$ allow for splitting to occur along each path in such a manner that weights do not increase. However, this can result in an unacceptable multiplication of the particle population. Carter and McCormick ${ }^{16}$ use a short Monte Carlo calculation of the neutron flux to bias the adjoint collision kernel. This has been demonstrated to be a useful technique in a production code, MCNA ${ }^{12}$ at LASL, but this method does suffer from the limitations that the additional transport calculation is required and that the necessary programming effort is significant.

In this section on the simulation of adjoint equations, we have discussed a number of advantages in solving certain problems with the adjoint approach. We have also attempted to point out that the adjoint simulation often requires special care to avoid unacceptably large variances. The investigation of effective methods of simulating the adjoint equation is presently an active area of research. 


\section{MULTIGROUP MONTE CARLO}

A multigroup treatment of the kinetic energy of the particles is sometimes used in Monte Carlo calculations of particle transport. To begin the discussion, we will consider a number of advantages and disadvantages of this approach over a continuousenergy treatment.

The computer programming tends to be simpler with a multigroup approach since cross sections and energy-transfer probabilities are cast in vector and matrix form. An advantage of this formulation is the similarity in the computer programs for simulating the transport of neutrons and gamma rays.

There is also a close correspondence between the simulation of the multigroup transport equation and its adjoint equation. This significantly reduces the computerprogramming effort when both modes of solution are required. However, the simulation of the adjoint equation may still suffer from large statistical errors for the reasons discussed in the previous section.

An important advantage of doing multigroup calculations with Monte Carlo is the generation of cross sections. Processor codes have been extensively developed in the past few years to generate cross sections for discrete-ordinates and diffusion-theory methods. These processor codes can be used with little or no alteration to generate cross sections for multigroup Monte Carlo codes.

Perhaps the most important advantage of this treatment is that it enables comparisons between Monte Carlo and discrete-ordinates calculations to be made directly with the identical cross-section sets. Thus the importance of geometrical approximations necessary to do a discrete-ordinates calculation can be evaluated.

The multigroup treatment does not necessarily assure a gain in the number of particle histories processed per unit of computation time. The Monte Carlo method is commonly used in geometrically complicated problems so that the tracking of particle histories through the geometry requires a significant fraction of the computation time. Thus the particular treatment of the collision process may not make a great deal of difference. In addition, the majority of the collisions are usually elastic, and the physics of elastic collisions is simple enough that they can be treated rapidly with a continuous-energy treatment.

Multigroup Monte Carlo has the obvious disadvantages inherent in group averaging. Thus it has definite limitations in problems where the fine energy detail of the cross sections is important, such as in treating resonance self-shielding problems.

Another disadvantage of the multigroup method involves the selection of the scattering angle at collision events. The usual multigroup approach is to expand the scattering kernel in a low-order Legendre expansion. The energy group of the emerging particle is selected in the random walk from the appropriate column of the $\mathrm{P}_{0}$ matrix. The density function for the scattering angle in the laboratory system is described by the corresponding components of the $\mathrm{P}_{1}, \mathrm{P}_{2}, \ldots, \mathrm{P}_{\mathrm{n}}$ matrices. Since this function represents the conditional angular distribution, given that a group-to-group scattering occurs, it may be highly anisotropic. Furthermore, the Legendre expansion is truncated so that the conditional angular distribution may actually go negative over a 
portion of the range. This is demonstrated in Fig. 4.3 for the in-group elastic scattering of neutrons on hydrogen nuclei for an energy group with the lower energy bound of the group equal to one-half the upper energy bound. The exact density function for the cosine of the scattering angle in the laboratory system is zero for $\mu<0.707$ and is shown in the figure for $\mu>0.707$.

The scattering angle can be sampled from the normalized density function

$$
\frac{|\mathrm{f}(\mu)|}{\int_{-1}^{1}\left|\mathrm{f}\left(\mu^{\prime}\right)\right| \mathrm{d} \mu^{\prime}}
$$

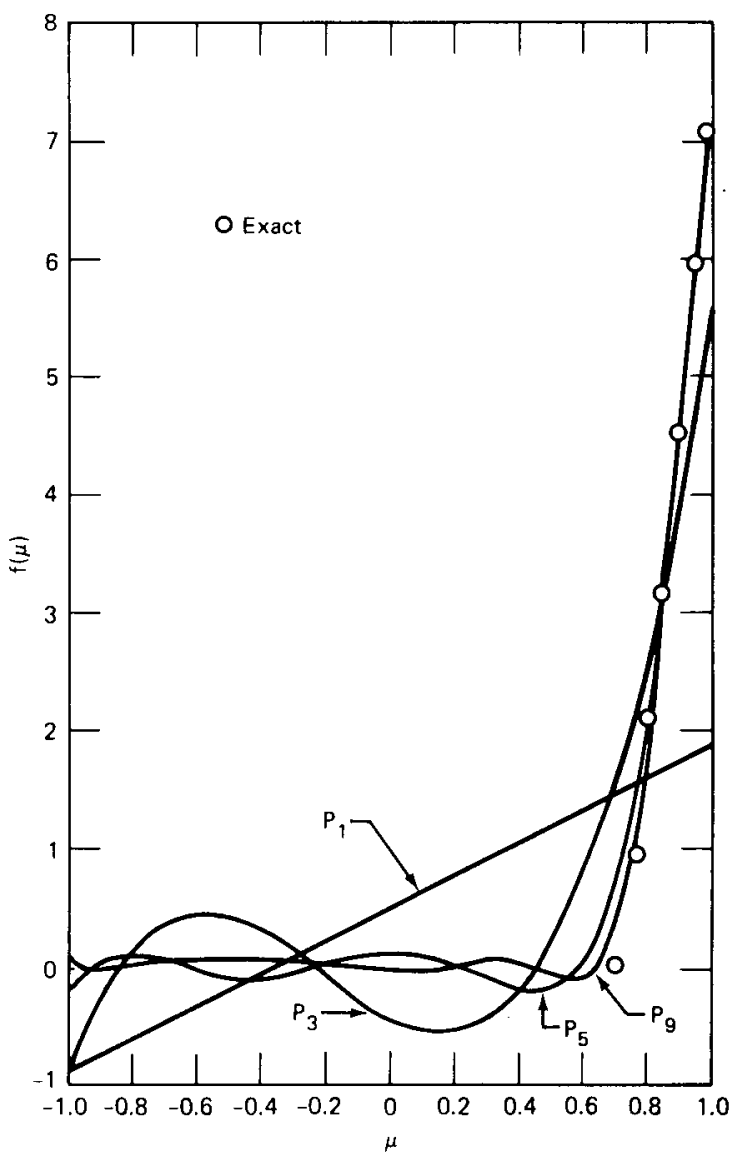

Fig. 4.3 In-group scattering density function for hydrogen. 
with the emerging particle weight adjusted by the factor

$$
\frac{\int_{-1}^{1}\left|\mathrm{f}\left(\mu^{\prime}\right)\right| \mathrm{d} \mu^{\prime}}{|\mathrm{f}(\mu)|} \mathrm{f}(\mu)
$$

to obtain an unbiased simulation of the multigroup equations. This weight-correction factor can result in a change in sign for the particle weight, which leads to statistical problems. It also has the disadvantage that even for the low-order expansion through $\mathrm{P}_{2}$ the density function is not trivial to sample.

A different technique for selecting the scattering angle is employed in the MORSE Monte Carlo code. ${ }^{4}$ A Gauss quadrature treatment is used to conserve the first few moments of the distribution. The particle is allowed to scatter into one of $n$ discrete scattering angles with $n$ corresponding probabilities. This technique requires $(2 n-1)$ words of data storage to conserve the first $(2 n-1)$ moments, i.e., $\left(\bar{\mu}_{1}, \bar{\mu}_{2}, \ldots, \bar{\mu}_{2 n-1}\right)$. This method avoids the negativity problem in most cases and is very fast to use in the random-walk process. However, it does suffer from the disadvantage that scattering is allowed only at discrete angles so the flux distribution tends to have some angular distortion during the first few collisions.

Spanier and Gelbard ${ }^{6}$ discuss a technique used in the MARC code. The scattering angle is selected from one of four distributions, i.e., straight forward, straight backward, at $90^{\circ}$, or from an isotropic distribution. The probabilities for selecting from the four distributions are computed to conserve the first four half-range moments.

The TART code 18,19 is a hybrid between a full multigroup treatment and a continuous-energy treatment. This code uses the reaction cross sections in multigroup form for each isotope. These include the total, capture, elastic, fission, inelastic cross sections for exciting discrete levels and the inelastic cross sections that correspond to available analytic or tabular data for the emerging neutron energy. The multigroup cross sections are used to sample flight paths and the type of collision event in the random walk. The emerging neutron energy and the scattering angle are then sampled by a continuous-energy treatment.

This discussion of multigroup methods has been brief. The interested reader should consult Refs. 4, 6, and 18 for a more detailed treatment and Ref. 20 for a comparison of various methods of sampling the scattering angle at collision events. In the next two chapters, we focus on techniques for sampling interaction models that are commonly used in describing neutron and photon transport.

REFERENCES

1. E. D. Cashwell and C. J. Everett, A Practical Manual on the Monte Carlo Method for Random Walk Problems, Pergamon Press, Inc., New York, 1959.

2. E. D. Cashwell, J. R. Neergaard, W. M. Taylor, and G. D. Turner, MCN: A Neutron Monte Carlo Code, USAEC Report LA-4751, Los Alamos Scientific Laboratory, 1972.

3. H. Lichtenstein and H. W. Bang, CG: Combinatorial Geometry Module for the Micom Laser Terminal Homing Systems Program, Report MR-7034, Mathematical Applications Group, Inc., 1973. 
4. E. A.Straker, W. H. Scott, Jr., and N. R. Byrn, MORSE General Purpose Monte Carlo Multigroup Neutron and Gamma-Ray Transport Code with Combinatorial Geometry, RSIC Computer Code Collection Report CCC-127D, Oak Ridge National Laboratory, 1972.

5. L. L. Carter, E. D. Cashwell, and W. M. Taylor, Monte Carlo Sampling with Continuously Varying Cross Sections A long Flight Paths, Nucl. Sci. Eng., 48:403-411 (1972).

6. J.Spanier and E. M. Gelbard, Monte Carlo Principles and Neutron Transport Problems, Addison-Wesley Publishing Company, Inc., Reading, Mass., 1969.

7. M. H. Kalos, On the Estimation of Flux at a Point by Monte Carlo, Nucl. Sci. Eng., 16:111 (1963).

8. H. A. Steinberg and M. H. Kalos, Bounded Estimators for Flux at a Point in Monte Carlo, Nucl. Sci. Eng., 44:406-412 (1971).

9. D. B. MacMillan, Comparison of Statistical Estimators for Neutron Monte Carlo Calculations, Nucl. Sci. Eng., 26:366-372 (1966).

10. E. M. Gelbard, L. A. Ondis II, and J.Spanier, A New Class of Monte Carlo Estimators, SIAM (Soc. Ind. Appl. Math.) J. Appl. Math., 14:697 (1966).

11. J. Spanier, Two Pairs of Families of Estimators for Transport Problems, SIAM (Soc. Ind. Appl. Math.) J. Appl. Math., 14:702 (1966).

12. L. L. Carter, MCNA: A Computer Program To Solve the Adjoint Neutron Transport Equation by Coupled Sampling with the Monte Carlo Method, USAEC Report LA-4488, Los Alamos Scientific Laboratory, 1971.

13. L. B. Levitt and J. Spanier, A New Non-Multigroup Adjoint Monte Carlo Technique, Nucl. Sci. Eng., 37:278-287 (1969).

14. D. C. Irving, The Adjoint Boltzmann Equation and Its Simulation by Monte Carlo, USAEC Report ORNL-TM-2870, Oak Ridge National Laboratory, 1970.

15. B. Eriksson, C. Johansson, M. Leimderfer, and M. H. Kalos, Monte Carlo Integration of the Adjoint Neutron Transport Equation, Nucl. Sci. Eng., 37:410 (1969).

16. L. L. Carter and N. J. McCormick, Coupled Sampling with the Monte Carlo Method in Neutron Transport Calculations, Nucl Sci. Eng., 39:296-310 (1970).

17. J. Spanier and G. J. Habetler, Application of Monte Carlo Methods to Neutron Thermalization, in Naval Reactor Physics Handbook, Vol. I, pp. 379-532, A. Radowsky (Ed.), USAEC Report TID-7030, Superintendent of Documents, U.S. Government Printing Office, Washington, 1964.

18. J. Kimlinger and E. F. Plechaty, SORS Monte Carlo Neutron-Transport Code for the CDC-6600, USAEC Report UCRL-50532, University of California Lawrence Livermore Laboratory, 1968.

19. E. F. Plechaty and J. R. Kimlinger, TART Monte Carlo Neutron-Transport Code, USAEC Report UCIR-522, University of California Lawrence Livermore Laboratory, 1971.

20. L. L. Carter and C. A. Forest, Transfer Matrix Treatments for Multigroup Monte Carlo Calculations-The Elimination of Ray Effects, Nucl. Sci. Eng. (accepted for publication; projected to appear in the January 1976 issue). 


\section{NEUTRON TRANSPORT}

\section{ELASTIC SCATTERING}

The mathematics describing the elastic scattering of a neutron from a free nucleus is considerably simplified if the precollision kinetic energy of the target nucleus can be neglected. This is usually a satisfactory approximation for neutron kinetic energies greater than about 10 times the equilibrium kinetic energy of the target nuclei. The case when the energy of the target nucleus is not negligible is discussed in the Thermal Scattering section of this chapter.

Elastic scattering is usually treated by selecting the scattering angle in the center-of-mass system and then computing the corresponding scattering angle in the laboratory system. This has the advantage of minimizing data-storage requirements since elastic scattering is isotropic in the center-of-mass system for a wide range of neutron energies. When incident-neutron energies are such that the scattering is not uniform, a computationally fast and accurate sampling method can be devised by tabulating the $(n+1)$ center-of-mass angles that correspond to $n$ equally probable intervals of the cumulative distribution function. Then in the random walk we randomly choose one of these intervals and sample the scattering angle from a uniform density function between the two scattering-angle bounds of the interval. The advantage of this technique is that the mesh points in the table are automatically dense where the probability density function is large. Data storage is also used effectively since we do not have to tabulate the cumulative distribution function; only the random variable bounds are needed. This is a special case of the sampling method discussed in the Basic Principle section of Chap. 2. The number $\mathrm{n}$ of equally likely intervals is typically chosen to be some integral power of 2 as a convenience for randomly sampling an interval on a binary machine. 
The density function for the scattering angle in the center-of-mass system depends in some continuous manner on the kinetic energy of the incident neutron, but satisfactory accuracy is obtained by tabulating at discrete incident-neutron energy points with some type of interpolation between these energy points. A commonly used method is linear interpolation of the density function in the following manner:

$$
\begin{aligned}
f\left(\mu_{c m} ; E\right)= & \left(\frac{E_{2}-E}{E_{2}-E_{1}}\right) f\left(\mu_{c m} ; E_{1}\right) \\
& +\left(\frac{E-E_{1}}{E_{2}-E_{1}}\right) f\left(\mu_{c m} ; E_{2}\right) \quad E_{1} \leqslant E \leqslant E_{2}
\end{aligned}
$$

where $f\left(\mu_{\mathrm{cm}} ; \mathrm{E}_{1}\right)$ is the density function at an incident-neutron energy $\mathrm{E}_{1}$ and $\mathrm{f}\left(\mu_{\mathrm{cm}} ; \mathrm{E}_{2}\right)$ is the density function at an incident-neutron energy $\mathrm{E}_{2}$. The density function is a linear combination of two density functions with positive coefficients; so a sampling technique suggested in the fourth section of Chap. 2 can be used, i.e., $\mu_{\mathrm{cm}}$ is sampled from $f\left(\mu_{c m} ; E_{1}\right)$ with probability $\left(E_{2}-E\right) /\left(E_{2}-E_{1}\right)$ or from $f\left(\mu_{c m} ; E_{2}\right)$ with probability $\left(E-E_{1}\right) /\left(E_{2}-E_{1}\right)$. The equally probable center-of-mass intervals are tabulated at $E_{1}$ and $E_{2}$. For example, the table for the energy $E_{1}$ would consist of the inverse solutions, $\left(\mu_{\mathrm{cm}}\right)_{\mathrm{i}}$, of the equation

$$
\frac{i}{n}=\int_{-1}^{\left(\mu_{c m}\right)_{i}} f\left(\mu_{c m} ; E_{1}\right) d \mu_{c m} \quad i=0,1,2, \ldots, n
$$

where $n$ is the number of equally likely intervals. Typically 16 or 32 intervals are adequate.

The corresponding scattering angle in the laboratory system is

$$
\mu_{\mathrm{lab}}=\frac{\left(1+\mathrm{A} \mu_{\mathrm{cm}}\right)}{\left(1+\mathrm{A}^{2}+2 \mathrm{~A} \mu_{\mathrm{cm}}\right)^{1 / 2}}
$$

and the emerging neutron energy is

$$
\mathrm{E}^{\prime}=(1 / 2) \mathrm{E}\left[(1-\alpha) \mu_{\mathrm{cm}}+1+\alpha\right]
$$

where

$$
\alpha=\left(\frac{A-1}{A+1}\right)^{2}
$$

and $A$ is the mass of the target nucleus in units of the mass of a neutron. 
The proton and neutron have essentially the same mass; so computational effort is saved by treating elastic scattering with a hydrogen nucleus separately. This is particularly true since the elastic scattering of a neutron with a proton is isotropic in the center-of-mass system for neutron energies less than about $10 \mathrm{MeV}$. In this energy range the event can be modeled simply by using one random number $\xi_{1}$ to select the emerging energy and the laboratory scattering angle as

$$
\mathrm{E}^{\prime}=\xi_{1} \mathrm{E}
$$

and

$$
\mu_{\mathrm{lab}}=\left(\xi_{1}\right)^{1 / 2}
$$

INELASTIC SCATTERING

The kinetic energy of the emerging neutron in the center-of-mass system after suffering an $\left(n, n^{\prime}\right)$ inelastic scattering is

$$
E_{c m}^{\prime}=\left(\frac{A}{A+1}\right)^{2} E+Q\left(\frac{A}{A+1}\right)
$$

where $\mathrm{E}$ is the incident kinetic energy of the neutron in the laboratory system, $\mathrm{Q}$ is the $Q$ value of the reaction, and the precollision kinetic energy of the target nucleus in the laboratory system has been neglected. The $Q$ value of the reaction is defined here as the rest energy of the target nucleus prior to the collision minus the rest energy of the target nucleus immediately after the collision (evaluated before the nucleus decays to its ground state). With this definition, $Q$ is always negative for the $\left(n, n^{\prime}\right)$ inelastic-scattering reaction being considered.

The scattering in the center-of-mass system is nearly isotropic for many cases of interest. If experimental measurements or theoretical calculations justify an anisotropic treatment, the center-of-mass scattering angle can be sampled in the random walk in the same manner we have discussed for elastic scattering.The emerging neutron energy in the laboratory system and the laboratory scattering angle are then determined from the center-of-mass scattering angle as

$$
\begin{gathered}
E^{\prime}=E_{c m}^{\prime}+\left[\frac{E+2 \mu_{c m}(A+1)\left(E E_{c m}^{\prime}\right)^{1 / 2}}{(A+1)^{2}}\right] \\
\mu_{1 a b}=\left(\frac{E_{c m}^{\prime}}{E^{\prime}}\right)^{1 / 2} \mu_{c m}+\left(\frac{E}{E^{\prime}}\right)^{1 / 2}\left(\frac{1}{A+1}\right)
\end{gathered}
$$


The appropriate score for the point-detector estimator discussed in Chap. 4, after excitation of an inelastic level as described above, will be derived. The right- and left-hand sides of Eq. (5.10) are squared, each term of the resulting equation is multiplied by $E^{\prime}$, and $E^{\prime}$ is then replaced by the expression given by Eq. (5.9). The result of this operation is a relation between $\mu_{\text {lab }}$ and $\mu_{\mathrm{cm}}$,

$$
\begin{gathered}
\mu_{\mathrm{lab}}^{2}\left\{\mathrm{E}_{\mathrm{cm}}^{\prime}+\left[\frac{\mathrm{E}+2 \mu_{\mathrm{cm}}(\mathrm{A}+1)\left(\mathrm{EE}_{\mathrm{cm}}^{\prime}\right)^{1 / 2}}{(\mathrm{~A}+1)^{2}}\right]\right\} \\
=\mu_{\mathrm{cm}}^{2} \mathrm{E}_{\mathrm{cm}}^{\prime}+\frac{2 \mu_{\mathrm{cm}}\left(\mathrm{E}_{\mathrm{cm}}^{\prime} \mathrm{E}\right)^{1 / 2}}{\mathrm{~A}+1}+\frac{\mathrm{E}}{(\mathrm{A}+1)^{2}}
\end{gathered}
$$

with $\mathrm{E}, \mathrm{E}_{\mathrm{cm}}^{\prime}$, and $\mathrm{A}$ constants for a given event. Therefore,

$$
\frac{\mathrm{d} \mu_{\mathrm{cm}}}{\mathrm{d} \mu_{\mathrm{lab}}}=\frac{\mu_{\mathrm{lab}} \mathrm{E}^{\prime}}{\mu_{\mathrm{cm}} \mathrm{E}_{\mathrm{cm}}^{\prime}+\left[\left(\mathrm{E}_{\mathrm{cm}}^{\prime} \mathrm{E}\right)^{1 / 2} /(\mathrm{A}+1)\right]-\left[\mu_{\mathrm{lab}}^{2}\left(\mathrm{E}_{\mathrm{cm}}^{\prime} \mathrm{E}\right)^{1 / 2} /(\mathrm{A}+1)\right]}
$$

which can be expressed as

$$
\frac{\mathrm{d} \mu_{\mathrm{cm}}}{\mathrm{d} \mu_{\mathrm{lab}}}=\frac{\left(\mathrm{E}^{\prime} / \mathrm{E}_{\mathrm{cm}}^{\prime}\right)}{\left(\mathrm{E}^{\prime} / \mathrm{E}_{\mathrm{cm}}^{\prime}\right)^{1 / 2}-\left[\mu_{\mathrm{lab}} /(\mathrm{A}+1)\right]\left(\mathrm{E} / \mathrm{E}_{\mathrm{cm}}^{\prime}\right)^{1 / 2}}
$$

by the use of Eq. (5.9) and (5.10). For a fixed scattering angle in the laboratory system, the final neutron energy can be obtained by eliminating $\mu_{\mathrm{cm}}$ from Eqs. (5.9) and (5.10) and solving for $E^{\prime}$ to obtain

$$
\left(E_{ \pm}^{\prime}\right)^{1 / 2}=\left[\frac{\mu_{1 a b}(E)^{1 / 2}}{A+1} \pm\left(\frac{\mu_{1 a b}^{2} E}{(A+1)^{2}}+E_{c m}^{\prime}-\frac{E}{(A+1)^{2}}\right)^{1 / 2}\right]
$$

There are three possibilities ${ }^{1}$ regarding the final energy of the neutron in the laboratory system determined by Eq. (5.14):

Case 1. If $\mathrm{E}_{\mathrm{cm}}^{\prime}<0$, which implies $\mathrm{E}<[(\mathrm{A}+1) / \mathrm{A}](-\mathrm{Q})$, then the quantity inside the right-hand square root of Eq. (5.14) is negative for all scattering angles in the laboratory system, and the reaction cannot occur. This corresponds to incident-neutron energies below threshold and hence is an uninteresting case.

Case 2. If $\mathrm{E}_{\mathrm{cm}}^{\prime}>\left[\mathrm{E} /(\mathrm{A}+1)^{2}\right]$, which implies $\mathrm{E}>[\mathrm{A} /(\mathrm{A}-1)](-\mathrm{Q})$, the quantity inside the square root is always greater than or equal to zero for all scattering angles in the laboratory system. However, the root corresponding to the positive sign 
in Eq. (5.14) is the only acceptable solution since the right-hand square root term is larger than $\left|(\mathrm{E})^{1 / 2} \mu_{\mathrm{ab}} /(\mathrm{A}+1)\right|$.

Case 3. If $0<\mathrm{E}_{\mathrm{cm}}^{\prime}<\mathrm{E} /(\mathrm{A}+1)^{2}$, or equivalently $[(\mathrm{A}+1) / \mathrm{A}](-\mathrm{Q})<$ $\mathrm{E}<[\mathrm{A} /(\mathrm{A}-1)](-\mathrm{Q})$, it is only possible to scatter through a range of scattering angles in the laboratory system such that $\left\{1-A^{2}-[Q A(A+1) / E]\right\}^{1 / 2}<\mu_{1 a b} \leqslant 1$. For any such acceptable scattering angle in the laboratory system, the neutron can emerge with either of the two energies corresponding to the roots of Eq. (5.14).

The point-detector score for each acceptable emerging neutron energy is given by

$$
\frac{\mathrm{Wh}\left(\mu_{\mathrm{cm}} ; \mathrm{E}\right)_{\mathrm{ik,cm}} \exp \left(-\int_{0}^{\left|\mathrm{r}-\mathrm{r}^{\prime}\right|} \Sigma_{\mathrm{t}} \mathrm{ds^{ \prime }}\right) \Sigma\left(\mathrm{R}^{\prime}\right)}{2 \pi\left|\mathrm{r}-\mathrm{r}^{\prime}\right|^{2}}\left|\frac{\mathrm{d} \mu_{\mathrm{cm}}}{\mathrm{d} \mu_{1 \mathrm{ab}}}\right|
$$

with $\mathrm{d} \mu_{\mathrm{cm}} / \mathrm{d} \mu_{\text {lab }}$ given by Eq. (5.13).

A few practical comments are in order. The possibility of multiple roots occurs when the kinetic energy of the incident neutron is just sufficient to excite the level. The significance of this observation is that the lower root $\mathrm{E}^{\prime}$ can usually be ignored without introducing appreciable error. In addition, subject to the previous consideration, $\mathrm{d} \mu_{\mathrm{cm}} / \mathrm{d} \mu_{\mathrm{lab}}$ is nearly unity for heavy nuclei, and the use of this approximation saves considerable computation effort and removes worry that the denominator of Eq. (5.13) may approach zero in rare cases. We return now to considerations of the random walk.

It is impossible to treat all energy levels for inelastic-scattering events. Furthermore, experimental measurements are usually either nonexistent or have been made for only a few of the lower levels. Hence some simple model is used to describe the majority of the levels. This model may consist of replacing a near continuum of $Q$ values with a number of discrete $Q$ values. Another model that enjoys considerable use is the statistical gas model, where the level structure is replaced by a continuum. The emerging-neutron energy is picked from the density function

$$
g\left(E^{\prime} ; E\right)=C_{1} E^{\prime} e^{-C_{2}(E) E^{\prime}} \quad\left(0 \leqslant E^{\prime} \leqslant E_{m}\right)
$$

where $C_{1}$ is a normalization constant, $C_{2}(E)$ depends on the properties of the nucleus of interest and on the incident-neutron energy, and $E_{m}$ is the maximum energy of the emitted neutrons. The density function $\mathrm{g}\left(\mathrm{E}^{\prime} ; \mathrm{E}\right)$ can be sampled as ${ }^{2}$

$$
E^{\prime}=\frac{-\ln \left(\xi_{1} \xi_{2}\right)}{C_{2}(E)}
$$

where $\mathrm{E}^{\prime}$ is accepted if $\mathrm{E}^{\prime}<\mathrm{E}_{\mathrm{m}}$. In $\mathrm{Eq} .(5.17), \xi_{1}$ and $\xi_{2}$ are random numbers on the unit interval. 
Various other methods are used to model $\left(n, n^{\prime}\right)$ inelastic-scattering events. The method used in the Monte Carlo calculation is often dictated by models that are recommended in the particular cross-section set being used.

The sampling of the inelastic-scattering events $(n, 2 n)$ and $(n, 3 n)$ is simplified by using models that ignore the correlation between the emerging neutrons. That is, the emerging kinetic energy and the scattering angle of each neutron are selected independently. This treatment yields unbiased results since on the average the correct amount of energy is emitted in each small energy interval even though energy is not conserved in the individual inelastic event. Of course, an exception to this occurs when quantities that depend on the correlation between the emerging neutrons are being estimated.

\section{FISSION}

The fission event is treated in a variety of ways, depending on the particular calculation of interest. For criticality calculations, usually only one generation (from one fission event to the next) of neutrons is followed for each fission-source iteration; so the neutrons produced at a fission event are banked for possible use in the subsequent iteration. For calculations in which the histories of the progeny are also of interest, the emerging neutrons from the fission event are sampled and followed in the random walk. Some care must be used in this description of the fission event for near-critical systems since it is possible for the Monte Carlo population of weighted histories to become supercritical even though the physical system is subcritical. This can be avoided by allowing less than $\nu$ neutrons to emerge from the fission event in the random walk and properly adjusting their weights. Carrying this to the extreme by allowing only one neutron to emerge from fission can introduce undesirable particle weight fluctuations in the random walk.

The sampling of the fission event is simplified by the usual assumption that the neutrons from fission are emitted isotropically in the laboratory system. In many computer codes the density function for the kinetic energy of the neutrons from a fission event is also assumed to be independent of the energy of the neutron causing fission. This is a useful approximation for nuclear-reactor calculations since only a small percentage of the neutrons from fission are emitted at energies above a few million electron volts. There are experimental measurements that indicate that, for fission events caused by an incident-neutron energy above a few million electron volts, one or two of the emergent neutrons may have been scattered inelastically rather than emitted in the fission process. The cross sections for these processes are denoted by $\sigma_{n, n^{\prime} f}$ and $\sigma_{n, 2 n^{\prime} f}$, respectively. This does not introduce any error in the number of neutrons emerging from the event since measurements of $\nu$ also include those neutrons scattered inelastically. However, it does introduce a change in the energy spectrum of the neutrons emitted from a fission event. The inclusion of these inelastic neutrons in the sampling is considered here. 
The cross section for the fission event, $\sigma_{n, F}$, can be expressed as

$$
\sigma_{n, F}=\sigma_{n, f}+\sigma_{n, n^{\prime} f}+\sigma_{n, 2 n^{\prime} f}
$$

where the partial cross sections $\sigma_{n, f}, \sigma_{n, n^{\prime}}$, and $\sigma_{n, 2 n^{\prime} f}$ are assumed to be available from experimental measurements or from theoretical calculations. Here $\sigma_{n, f}$ is the cross section for true fission, where no inelastic neutrons are emitted in addition to those from the fission process. Such data for ${ }^{235} \mathrm{U},{ }^{238} \mathrm{U},{ }^{239} \mathrm{Pu}$, and ${ }^{240} \mathrm{Pu}$, along with fits to the spectra of emerging neutrons for the various reactions, are available in Refs. 3 and 4.

Experimental measurements indicate that the kinetic-energy spectrum of the neutrons emitted in the true fission process is unaffected by the inelastically scattered neutrons. Hence the energy of an emerging neutron from the fission event is selected from the inelastic spectrum of the ( $\left.n, n^{\prime} f\right)$ event with probability

$$
\frac{\sigma_{\mathrm{n}, \mathrm{n}^{\prime} \mathrm{f}}}{\nu \sigma_{\mathrm{n}, \mathrm{F}}}
$$

and from the inelastic spectrum of the ( $\left.n, 2 n^{\prime} f\right)$ event with probability

$$
\frac{2 \sigma_{n, 2 n^{\prime} f}}{\nu \sigma_{n, F}}
$$

If the random test does not select either of these two possibilities, the emerging-neutron energy is sampled from a fission spectrum.

A fission spectrum can be handled in the Monte Carlo calculation by tabulating equally probable energy bins for the sampling. Alternatively, we can sample from an analytic fit, as discussed in Chap. 2.

\section{THERMAL SCATTERING}

Neutron scattering at thermal energies is modeled with a variety of methods. Scattering can be treated in a rigorous manner by sampling directly for $\alpha$ and $\beta$ from the $S(\alpha, \beta)$ scattering law this also includes chemical-binding effects. The $\alpha$ and $\beta$ uniquely determine the kinetic energy and scattering angle of the emerging neutron in the laboratory system. This direct sampling of $\alpha$ and $\beta$ enables calculations to be made with the full scattering kernel but requires the storage of only two-dimensional arrays. A formulation for sampling $\alpha$ and $\beta$ is discussed in detail in Ref. 5.

Computer storage limitations, computational speed requirements, and the fact that chemical binding may often be either ignored or adequately described by various approximations have led to the extensive use of simpler thermal models in Monte 
Carlo codes. An approximation that is often used is a multigroup treatment of the thermal energy range. This is quite effective since the thermal target motion tends to make the scattering more nearly isotropic so that a low-order Legendre expansion of the frequency distribution for the scattering angle is satisfactory for many applications.

The free gas kernel is a thermal interaction model that enjoys considerable use since it results in a good approximation to the thermal flux spectrum in a variety of applications and can be sampled without tables. In this model neutrons are assumed to be transported in a monatomic gas, the latter having an isotropic Maxwellian distribution of velocities. The effective scattering cross section in the laboratory system for a neutron of kinetic energy $E$ is

$$
\sigma_{\mathrm{s}}^{\mathrm{eff}}(\mathrm{E})=\frac{1}{\mathrm{v}_{\mathrm{n}}} \iint \sigma_{\mathrm{s}}\left(\mathrm{v}_{\mathrm{rel}}\right) \mathrm{v}_{\mathrm{rel}} \mathrm{p}\left(\mathrm{V}, \mu_{\mathrm{t}}\right) \mathrm{dV} \mathrm{d} \mu_{\mathrm{t}}
$$

Here, $v_{\text {rel }}$ is the relative velocity between a neutron moving with a scalar velocity $v_{n}$ and a target nucleus moving with a scalar velocity $V$, and $\mu_{t}$ is the cosine of the angle between the neutron and the target direction-of-flight vectors. The scattering cross section for this relative velocity is denoted by $\sigma_{s}\left(v_{\text {rel }}\right)$, and $p\left(V, \mu_{t}\right)$ is the probability density function for the Maxwellian distribution of target velocities,

$$
p\left(V, \mu_{t}\right)=\frac{2}{\pi^{1 / 2}} \beta^{3} V^{2} e^{-\beta^{2} V^{2}}
$$

with $\beta$ defined as

$$
\beta=\left(\frac{\mathrm{A}}{2 \mathrm{kT}}\right)^{1 / 2}
$$

In Eq. (5.23) $\mathrm{A}$ is the mass of a target nucleus in units of the mass of a neutron, $\mathrm{k}$ is the Boltzmann constant, and $\mathrm{T}$ is the equilibrium temperature of the target nuclei.

The relative velocity between the neutron and target is obtained from the cosine law,

$$
v_{\text {rel }}=\left[\left(v_{n}\right)^{2}+v^{2}-2 v_{n} v \mu_{t}\right]^{1 / 2}
$$

We assume that the scattering cross section of a nucleus is independent of this relative velocity; so

$$
\sigma_{s}\left(v_{\text {rel }}\right)=\sigma_{s}^{0}=\text { constant }
$$


This assumption enables us to evaluate the integral over $\mu_{\mathrm{t}}$ in Eq. (5.21) and to express the scattering cross section in the laboratory system as

$$
\begin{aligned}
\sigma_{\mathrm{s}}^{\text {eff }}(\mathrm{E}) & =\frac{1}{\mathrm{v}_{\mathrm{n}}} \int_{0}^{\infty} \frac{2 \sigma_{\mathrm{s}}^{0} \beta^{3} \mathrm{~V}^{2} \mathrm{e}^{-\beta^{2} \mathrm{~V}^{2}}}{\pi^{1 / 2}}\left\{\int_{-1}^{1}\left[\left(\mathrm{v}_{n}\right)^{2}+\mathrm{V}^{2}-2 \mathrm{v}_{\mathrm{n}} \mathrm{V} \mu_{\mathrm{t}}\right]^{1 / 2} \mathrm{~d} \mu_{\mathrm{t}}\right\} \mathrm{dV} \\
& =\frac{2 \sigma_{\mathrm{s}}^{0}}{3 \pi^{1 / 2}\left(\mathrm{v}_{\mathrm{n}}\right)^{2}} \int_{0}^{\infty}\left[\left(\mathrm{v}_{\mathrm{n}}+\mathrm{V}\right)^{3}-\left|\mathrm{v}_{\mathrm{n}}-\mathrm{V}\right|^{3}\right] \beta^{3} \mathrm{~V} \mathrm{e}^{-\beta^{2} \mathrm{~V}^{2}} \mathrm{dV}
\end{aligned}
$$

It is convenient in the following considerations to define a dimensionless variable $\mathrm{x}$ as

$$
\mathrm{x}=\beta \mathrm{V}
$$

so that $\mathrm{x}^{2}$ is the ratio of the kinetic energy of a target nucleus to kT. We also define the constant a as

$$
\mathrm{a}=\beta \mathrm{v}_{n}
$$

Then, with a change of variables, the effective scattering cross section is

$$
\sigma_{s}^{e f f}(E)=\frac{2 \sigma_{s}^{0}}{3 a^{2} \pi^{1 / 2}} \int_{0}^{\infty}\left[(a+x)^{3}-|a-x|^{3}\right] x e^{-x^{2}} d x
$$

which is equivalent to the well-known result

$$
\sigma_{s}^{\text {eff }}(E)=\sigma_{s}^{0}\left[\left(1+\frac{1}{2 a^{2}}\right) \operatorname{erf}(a)+\frac{1}{a \pi^{1 / 2}} e^{-a^{2}}\right]
$$

The integrand on the right-hand side of Eq. (5.29) is proportional to the probability density function for the target velocity in the transformed $x$ variable. This density function for $\mathrm{x}$ decreases in value rapidly as $\mathrm{x}$ becomes large; so the total probability of $x>3$ can be shown to be less than 0.00125 . The random walk is simplified by neglecting $\mathrm{x}>3$, in which case an $\mathrm{x}$ value is sampled uniformly between zero and 3 and accepted with a probability proportional to the integrand on the right-hand side of Eq. (5.29). The efficiency of such a sampling is approximately 0.4 , and this efficiency is nearly independent of the incident-neutron energy. The approximation involved in neglecting $x>3$ is equivalent to ignoring all target energies greater than $9 \mathrm{kT}$. 
We also observe from Eq. (5.26) that the density function for the cosine of the angle between the target and neutron direction-of-flight vectors is given by

$$
f\left(\mu_{t}\right)=C\left(a^{2}+x^{2}-2 a x \mu_{t}\right)^{1 / 2}
$$

This can be sampled analy tically as

$$
\mu_{t}=\frac{1}{2 a x}\left(a^{2}+x^{2}-\left\{|a-x|^{3}-\xi\left[|a-x|^{3}-(a+x)^{3}\right]\right\}^{2 / 3}\right)
$$

The incident-neutron direction of flight will be denoted here by the direction-of-flight cosines $(\mathrm{u}, \mathrm{v}, \mathrm{w})$. This incident direction, the value of $\mu_{t}$, and an azimuth angle uniformly selected on the cone about the incident direction of flight of the neutron are sufficient to fix the direction of flight of the target nucleus, denoted here by the direction cosines $\left(\mathrm{u}_{\mathrm{t}}, \mathrm{v}_{\mathrm{t}}, \mathrm{w}_{\mathrm{t}}\right)$.

The scattering is assumed to be isotropic in the center-of-mass system; so the new direction of flight in this system of coordinates is sampled uniformly on the unit sphere and is denoted by the direction cosines $\left(\mathrm{u}_{\mathrm{o}}, \mathrm{v}_{\mathrm{o}}, \mathrm{w}_{\mathrm{o}}\right)$. The kinematics of the collision process ${ }^{6}$ yields the final neutron energy and direction-of-flight cosines in the laboratory system as

$$
\begin{aligned}
& E^{\prime}=\frac{E}{(A+1)^{2}}\left(\bar{x}^{2}+\bar{y}^{2}+\bar{z}^{2}\right) \\
& u^{\prime}=\frac{\bar{x}}{\left(\bar{x}^{2}+\bar{y}^{2}+\bar{z}^{2}\right)^{1 / 2}} \\
& v^{\prime}=\frac{\bar{y}}{\left(\bar{x}^{2}+\bar{y}^{2}+\bar{z}^{2}\right)^{1 / 2}} \\
& w^{\prime}=\frac{\bar{z}}{\left(\bar{x}^{2}+\bar{y}^{2}+\bar{z}^{2}\right)^{1 / 2}}
\end{aligned}
$$

where

$$
\begin{aligned}
& \bar{x}=u+A\left(\delta u_{0}+\frac{x}{a} u_{t}\right) \\
& \bar{y}=v+A\left(\delta v_{0}+\frac{x}{a} v_{t}\right)
\end{aligned}
$$




$$
\begin{aligned}
& \bar{z}=w+A\left(\delta w_{0}+\frac{x}{a} w_{t}\right) \\
& \delta=\left(1+\frac{x^{2}}{a^{2}}-\frac{2 x \mu_{t}}{a}\right)^{1 / 2}
\end{aligned}
$$

Figure 5.1 is a flow diagram for sampling the free gas kernel, based on the above derivation. In the figure, $E$ is the precollision kinetic energy of the neutron, and $(\mathrm{u}, \mathrm{v}, \mathrm{w})$ are the precollision direction-of-flight cosines. The postcollision kinetic energy of the neutron is denoted by $E^{\prime}$, and the postcollision direction-of-flight cosines are denoted by $\left(\mathrm{u}^{\prime}, \mathrm{v}^{\prime}, \mathrm{w}^{\prime}\right)$.

In some applications we find it convenient to use the point-detector estimator discussed in Chap. 4 in conjunction with the free gas kernel. We describe here a method to score at the point detector for each collision event. For the $\left(x, \mu_{t}\right)$ selected from the scheme of Fig. 5.1 in the random walk, the kinetic energy that the neutron must have in order to scatter to the detector can be obtained from energy and momentum balances. The resulting equations are straightforward to derive, but we have omitted the rather involved mathematical details. A flow diagram for the point-detector score is given in Fig. 5.2, assuming $(x, y, z)$ is the point of collision and $\left(\mathrm{x}^{\prime}, \mathrm{y}^{\prime}, \mathrm{z}^{\prime}\right)$ is the location of the detector.

\section{CRITICALITY}

Criticality calculations have the distinguishing feature that the equilibrium spatial-source distribution of the fission neutrons is unknown at the beginning of the calculation. Sometimes an adequate source distribution can be constructed from deterministic methods, but, in the more common situation, it must also be constructed in the course of the Monte Carlo calculation. The computation time required to obtain a converged source is sometimes an unimportant consideration (e.g., for fast critical assemblies), but it is often a significant factor in determining the feasibility of doing Monte Carlo calculations on thermal systems. There are also physical systems, such as certain array configurations of fissionable materials and split-core reactors, where care must be exercised to ensure that the iteration method will converge to the correct equilibrium source distribution.

The typical Monte Carlo calculation uses an initial neutron source distribution from fission which is either obtained from a simplified deterministic calculation or is merely a guess. This initial distribution is used as the neutron source for the first generation of neutrons in the Monte Carlo calculation. The resulting progeny from fission events is used to construct the neutron source for the next generation, and so the iteration proceeds. After a sufficient number of generations, convergence of the fission-source distribution is deemed satisfactory from some test, and the neutron histories in a number of subsequent generations are used to compute quantities of interest, such as the multiplication factor. 

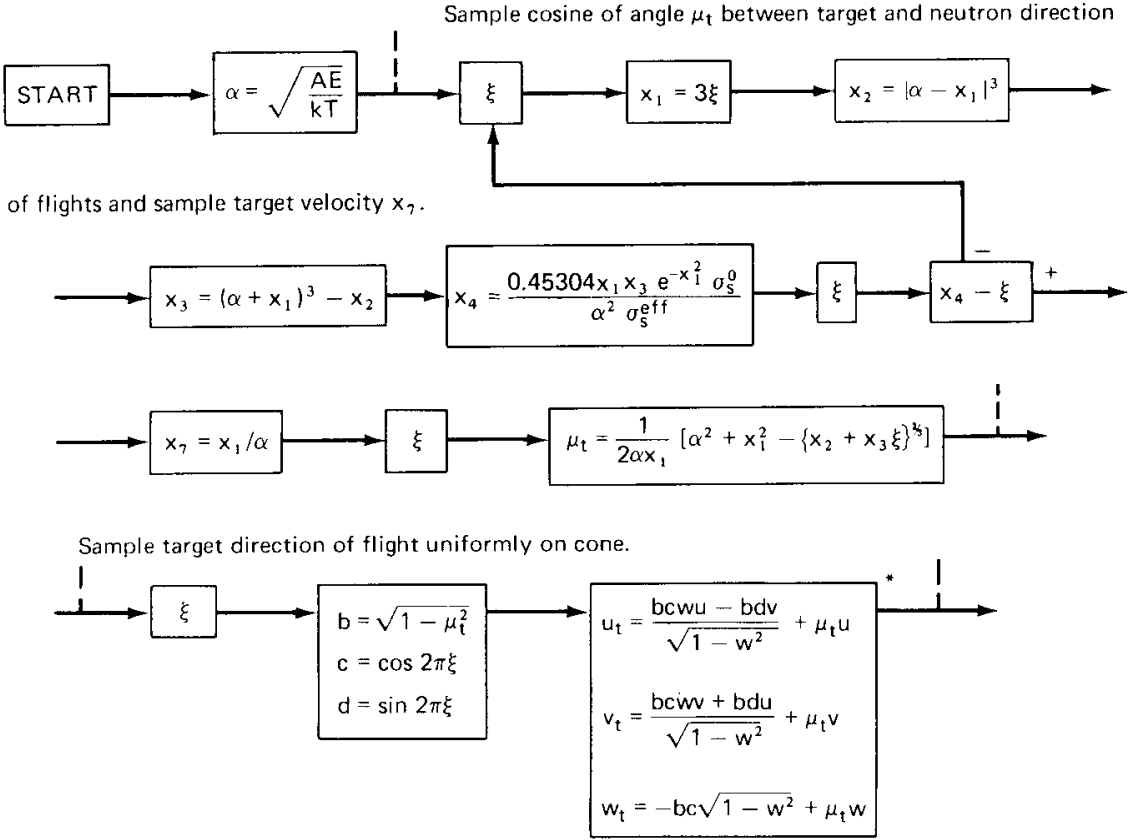

Sample random direction assuming isotropic scattering in center of mass.
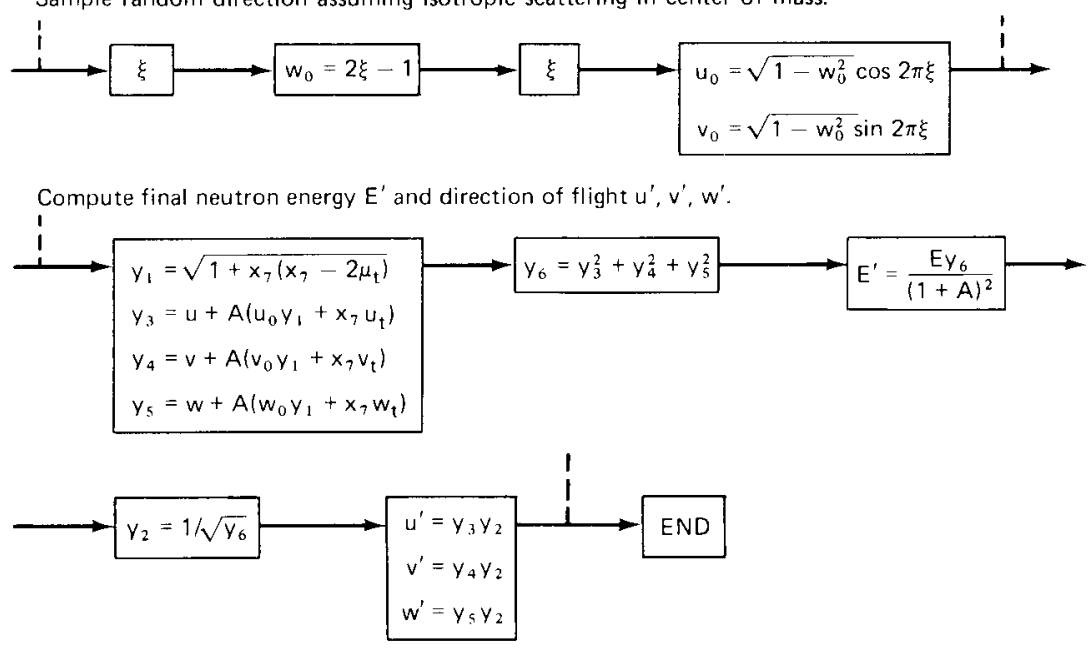

*Use correct limits ${ }^{6}$ as $j \mathrm{w} \mid \rightarrow 1$.

Fig. 5.1 Flow diagram for sampling the free gas kernel. 
We can reduce the computation time required for source convergence by replacing the Monte Carlo iteration on the source with a fission matrix iteration. The volume of the system containing fissionable material is divided into a number of contiguous spatial cells, and an element $a_{i j}$ of the matrix is defined to be numerically equal to the number of first-generation fission neutrons produced in cell i from one fission neutron starting in cell $\mathrm{j}$. These elements can be computed in a one-generation Monte Carlo calculation. The subsequent numerical iteration using the matrix with an initial source vector typically requires a negligible amount of computational effort.

The disadvantage of the matrix method is that the elements themselves are computed from an assumed initial distribution in the Monte Carlo calculation. The error due to this initial distribution tends to be of second order since only the functional dependence of the fission source across individual cells, rather than across the entire system, is important for computing the matrix elements. If the error due to the assumed distribution across individual cells does turn out to be significant, it can be reduced by using information from the first matrix iteration to repeat and improve the Monte Carlo calculation of the matrix elements. The statistical error involved in the evaluation of the matrix elements also leads to an error in the eigenvalue obtained from the matrix iteration. Recent studies have indicated that this is an important disadvantage of a matrix method.

The interested reader is referred to Ref. 7 for examples in which Monte Carlo was used to iterate the fission-source distribution. Fission-source iterations with a matrix approach are discussed in Refs. 8 and 9; these two references are also excellent as an introduction to the use of Monte Carlo in reactor-physics problems. Techniques to improve and accelerate the matrix method are discussed in Ref. 10.

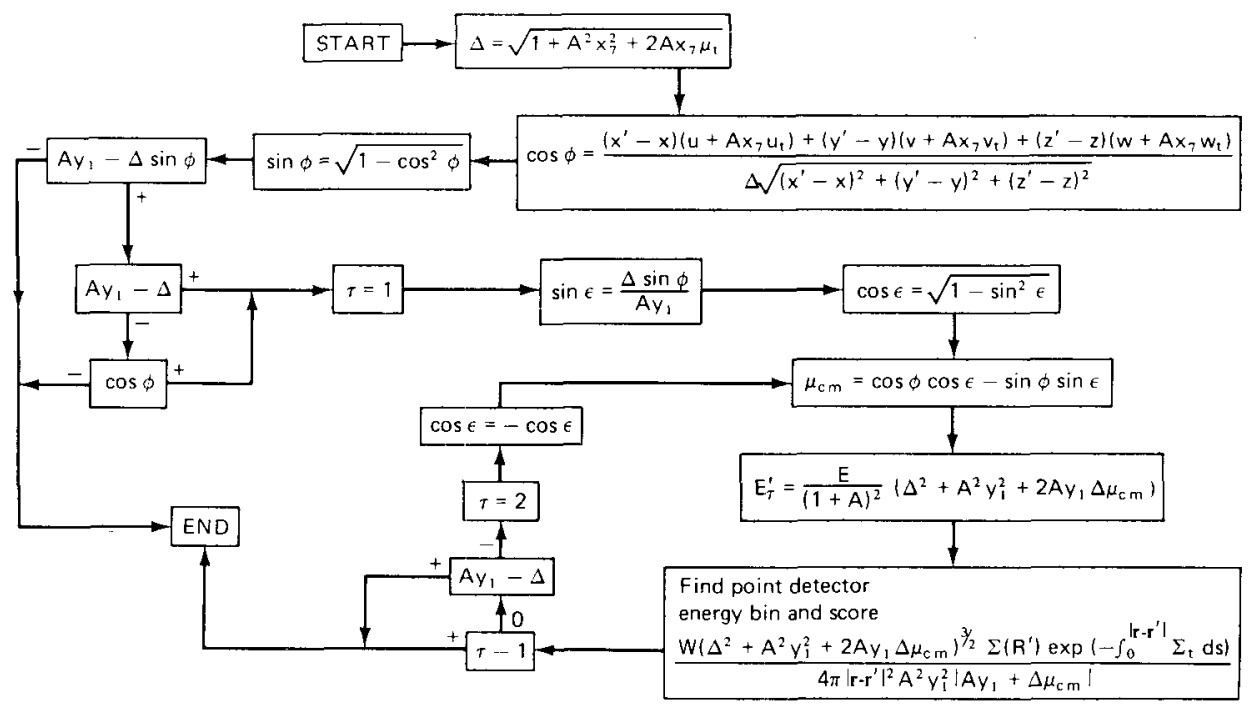

Fig. 5.2 Point-detector score in the thermal routine. 


\section{REFERENCES}

1. C. J. Everett and E. D. Cashwell, Scattering Formulas for the Two-Particle Reaction, USAEC Report LA-5196-MS, Los Alamos Scientific Laboratory, 1973.

2. C. J. Everett and E. D. Cashwell, A Monte Carlo Sampler, USAEC Report LA-5061-MS, Los Alamos Scientific Laboratory, 1972.

3. J. J. Berlijn, R. E. Hunter, and C. C. Cremer, Neutron Cross Sections for ${ }^{235} \mathrm{U}$ and ${ }^{238} \mathrm{U}$ in the Energy Range $1 \mathrm{keV}$ to $14 \mathrm{MeV}$, USAEC Report LA-3527, Los Alamos Scientific Laboratory, 1968.

4. R. E. Hunter, J. J. Berlijn, and C. C. Cremer, Neutron Cross Sections for ${ }^{239} \mathrm{Pu}$ and ${ }^{240} \mathrm{Pu}$ in the Energy Range $1 \mathrm{keV}$ to $14 \mathrm{MeV}$, USAEC Report LA-3528, Los Alamos Scientific Laboratory, 1968.

5. F. G. Bischoff, M. L. Yeater, and W. E. Moore, Monte Carlo Evaluation of Multiple Scattering and Resolution Effects in Double-Differential Neutron Scattering Cross-Section Measurements, Nucl. Sci. Eng., 48: 266-280 (1972).

6. E. D. Cashwell and C. J. Everett, A Practical Manual on the Monte Carlo Method for Random Walk Problems, Pergamon Press, Inc., New York, 1959.

7. J. T. Mihalczo, Criticality of Graphite- and Polyethylene-Reflected Uranium (93.2\%)-Metal Cylinders and Annuli, Nucl. Sci. Eng., 49: $489-504$ (1972).

8. M. R. Mendelson, Monte Carlo Criticality Calculations for Thermal Reactors, Nucl. Sci. Eng., 32: 319-331 (1968).

9. M. H. Kalos, F. R. Nakache, and J. Celnik, Monte Carlo Methods in Reactor Computations, in Computing Methods in Reactor Physics, pp. 359-438, H. Greenspan, C. N. Kelber, and D. Okrent (Eds.), Gordon and Breach, Science Publishers, Inc., New York, 1968.

10. L. L. Carter and N. J. McCormick, Source Convergence in Monte Carlo Calculations, $\mathrm{Nucl}$. Sci. Eng., 36: 438-441 (1969). 


\section{PHOTON TRANSPORT}

INTRODUCTION

Since photons can be considered as particles subject to scattering laws as they pass through matter in much the same manner as neutrons, the techniques of Monte Carlo are eminently suited to photon-transport problems. 'A linear equation governing photon transport is essentially identical in form to the Boltzmann transport equation for neutrons. The collision processes of photons on electrons, although physically quite different from those of neutrons, are analogous to corresponding processes of neutrons. In this chapter, we discuss the principal types of collision and ways of describing them by Monte Carlo.

\section{COMPTON COLLISIONS}

A Compton collision is by definition a collision of a photon with an electron that is assumed to be free and at rest in the laboratory system of coordinates. We use the following definitions in this chapter:

$$
\begin{aligned}
c & =2.997925 \times 10^{10} \mathrm{~cm} / \mathrm{sec}, \text { the velocity of light in a vacuum } \\
\mathrm{h} & =6.626196 \times 10^{-27} \mathrm{erg} \mathrm{sec}, \text { Planck's constant } \\
\mathrm{m}_{0} & =9.109558 \times 10^{-28} \mathrm{~g}, \text { the rest mass of the electron } \\
\mathrm{e} & =4.803251 \times 10^{-10} \mathrm{esu}, \text { the charge of the electron }
\end{aligned}
$$


In the treatment of Compton collisions, the photon has an energy $E$ that satisfies the relation $\mathrm{E}=\mathrm{h} \nu$, where $\nu$ is the frequency of the photon. The particle also has a wavelength $\lambda$, with $\lambda \nu=c$. A mass can be defined for a photon by means of the relation $\mathrm{E}=\mathrm{mc}^{2}$, and a momentum $\mathrm{mV}$ can be assigned, with $|\mathbf{V}|=\mathrm{c}$ and $|\mathrm{mV}|=\mathrm{mc}=\mathrm{h} \nu / \mathrm{c}$.

The electron in these collisions is characterized by its charge e, rest mass $m_{0}$, and velocity vector $\mathbf{V}^{\prime}$. Its mass is then $m=m_{0} /\left(1-\beta^{2}\right)^{1 / 2}$, with $\beta=\left|\mathbf{V}^{\prime}\right| / \mathrm{c}$. The momentum is $m V^{\prime}$, and its total energy is $m c^{2}$.

Many of the relations derived from considering photons colliding with free electrons are simplified if the photon energies are expressed by a dimensionless parameter $\alpha=h \nu / \mathrm{m}_{0} \mathrm{c}^{2}$, which gives the ratio of the photon energy to the rest energy of the electron, $\mathrm{m}_{0} \mathrm{c}^{2}=0.511004 \mathrm{MeV}\left(1 \mathrm{MeV}=1.60210 \times 10^{-6} \mathrm{erg}\right)$.

In a Compton collision the total energy and momentum are preserved. Using the foregoing definitions and writing down these conservation laws, we can derive the following relation between the incoming photon energy $\alpha$, the scattering angle, $\theta$, and the outgoing photon energy, $\alpha^{\prime}$ :

$$
\alpha^{\prime}=\frac{\alpha}{1+\alpha(1-\mu)}
$$

Here $\mu=\cos \theta$, the cosine of the scattering angle in the laboratory system, and the energies $\alpha$ and $\alpha^{\prime}$ are in units of the rest energy of the electron, $\mathrm{m}_{0} \mathrm{c}^{2}$. The pertinent information on the electron, such as the scattering angle and the energy, is immediate from the derivation. The interested reader may consult Chap. VI of the manual by Cashwell and Everett ${ }^{1}$ for the derivation of these relations.

The Klein-Nishina cross section for the scattering of a photon of energy $\alpha=$ $\mathrm{E} / \mathrm{m}_{0} \mathrm{c}^{2}$ on a free electron at rest, at an angle $\theta$ within $\mathrm{d} \mu$ of $\mu=\cos \theta$ from its line of flight, is given by

$$
\sigma(\alpha, \mu) \mathrm{d} \mu=\pi \mathrm{r}_{0}^{2}\left(\frac{\alpha^{\prime}}{\alpha}\right)^{2}\left(\frac{\alpha^{\prime}}{\alpha}+\frac{\alpha}{\alpha^{\prime}}+\mu^{2}-1\right) \mathrm{d} \mu \quad(-1 \leqslant \mu \leqslant 1)
$$

where $\alpha^{\prime}=\alpha /[1+\alpha(1-\mu)]$ is its final energy $\alpha^{\prime}=E^{\prime} / m_{0} c^{2}$, and $r_{0}=e^{2} / m_{0} c^{2}=$ $2.81794 \times 10^{-13} \mathrm{~cm}$ is the classical Thomson radius of the electron.

With the incoming energy $\alpha$ fixed, define $\mathrm{x}=\alpha^{\prime} / \alpha=1 /[1+\alpha(1-\mu)]$ and $\widetilde{\sigma}(\alpha, \mathrm{x}) \mathrm{dx} \equiv \sigma(\alpha, \mu) \mathrm{d} \mu$. Writing Eq. 6.1 in the form $\mu=1+\alpha^{-1}-\alpha^{-1} \mathrm{x}^{-1}$, we have $\mathrm{d} \mu / \mathrm{dx}=\alpha^{-1} \mathrm{x}^{-2}$ and

$$
\widetilde{\sigma}(\alpha, x) d x=\pi r_{0}^{2} \alpha^{-1}\left(x+x^{-1}+\mu^{2}-1\right) d x
$$

The expression on the right has an associated probability density function

$$
p(x) d x=\frac{f(x) d x}{F(\beta)}
$$




$$
\beta \equiv \frac{1}{2 \alpha+1} \leqslant x \leqslant 1
$$

where

$$
\begin{aligned}
f(x) & =x+x^{-1}+\mu^{2}-1 \\
\mu & =1+\alpha^{-1}-\alpha^{-1} x^{-1} \\
F(\beta) & =\int_{\beta}^{1} f(x) d x \equiv G
\end{aligned}
$$

The Monte Carlo method of sampling for $\mathrm{x}=\alpha^{\prime} / \alpha$ consists in solving the equation

$$
\begin{aligned}
\xi & =\frac{F(x)}{G} \\
F(x) & =\int_{x}^{1} f(t) d t
\end{aligned}
$$

for $\mathrm{x}$ in terms of a random number $\xi$, equidistributed on $[0,1]$.

Referring to Eqs. 6.3, 6.5, and 6.6, we can compute the Compton scattering cross section from the relation

$$
\sigma_{\alpha}(\text { Compton })=\pi \mathrm{r}_{0}^{2} \alpha^{-1} \mathrm{G}
$$

This cross section has been plotted by the National Bureau of Standards. ${ }^{2}$

Various methods have been used to sample the Klein-Nishina scattering function. Very early in the development of Monte Carlo, $\mathrm{Kahn}^{3}$ devised a rejection technique to sample this function. Another method that has proved very successful in practice is to approximate the inverse function $x=F^{-1}(y) \cong Q(y)$ of $y=F(x)$ given in Eq. 6.7 and to take $\mathrm{x}=\mathrm{F}^{-1}(\mathrm{G} \xi) \cong \mathrm{Q}(\mathrm{G} \xi)$. The method can be fast to use since each random number leads to a value of $x$. Examples of this latter scheme appear in the literature, ${ }^{4,5}$ where very accurate fits to the inverse function are displayed. Both the rejection method and the approximation of the inverse function have been incorporated into machine codes for transporting photons. Some of these codes are mentioned later in this chapter.

More accurate treatment of the photon-scattering process necessitates consideration of the structure of the atom and leads to two types of scattering, incoherent and coherent.

\section{INCOHERENT SCATTERING}

Scattering from free electrons is referred to as incoherent since the independent behavior of the electrons prevents any interference effects. In this process the differential scattering cross section is written in the form 


$$
\sigma^{\mathrm{i}}(\mathrm{Z}, \alpha, \mu) \mathrm{d} \mu=\mathrm{I}(\mathrm{Z}, \mathrm{v}) \mathrm{K}(\alpha, \mu) \mathrm{d} \mu
$$

where $\mathrm{I}(\mathrm{Z}, \mathrm{v})$ is an appropriate scattering factor modifying the Klein-Nishina cross section

$$
\mathrm{K}(\alpha, \mu) \mathrm{d} \mu=\pi \mathrm{r}_{0}^{2}\left(\frac{\alpha^{\prime}}{\alpha}\right)^{2}\left(\frac{\alpha^{\prime}}{\alpha}+\frac{\alpha}{\alpha^{\prime}}+\mu^{2}-1\right) \mathrm{d} \mu
$$

Here $\mathrm{K}(\alpha, \mu)$ is written in place of $\sigma(\alpha, \mu)$ in Eq. 6.2. As in the latter equation, $\alpha^{\prime}=\alpha /[1+\alpha(1-\mu)]$, with the definitions of $\mathrm{r}_{0}$ and $\mu$ the same as before. The scattering factor represents the probability that an atomic electron, having been imparted momentum by a photon, will absorb energy and thereby become excited or leave the atom.

The factor $I(Z, v)$ has the effect of decreasing the Klein-Nishina cross section more extremely in the forward direction for low $E$ and for high $Z$ independently; it increases from $I(Z, 0)=0$ to $I(Z, \infty)=Z$. The parameter $v=v(\alpha, \mu)$ is a function of $\alpha$ and $\mu$ which, for a given value of the incident energy $\alpha$, increases from $v(\alpha, 1)=0$ at $\mu=1$ to a maximum value $\overline{\mathrm{v}}=\mathrm{v}(\alpha,-1)$ at $\mu=-1$. As $\mu$ varies from 1 to -1 , the quantity $I(Z, v) / Z$ increases rapidly from 0 toward 1 . At high energies $I(Z, v) / Z$ is approximately 1 except for the extreme forward direction. The variation of $I(Z, v) / Z$ with $v$ for different values of $Z$ is shown in Fig. 6.1. Here the parameter $v$ is the inverse length $\mathrm{v}=\sin (1 / 2) \theta / \lambda(\AA)=\kappa \alpha(1-\mu)^{1 / 2}, \kappa=10^{-8}, \mathrm{~m}_{0} \mathrm{c} / \mathrm{h}(2)^{1 / 2}=29.1433 \mathrm{~cm}^{-1}$, with maximum value $\bar{v}=\kappa \alpha(2)^{1 / 2}$ for given $\alpha$.

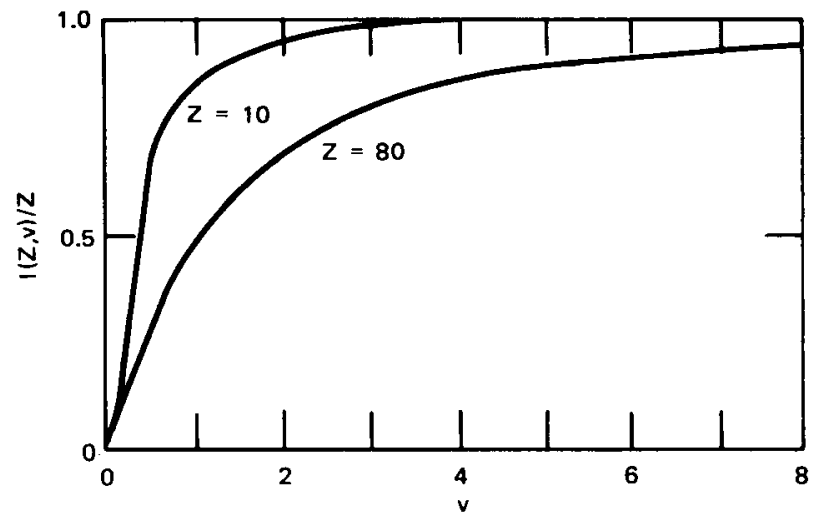

Fig. 6.1 Incoherent scattering factor. 
To describe a sampling method for $\mu=\cos \theta$ from $\mathrm{I}(Z, v) \mathrm{K}(\alpha, \mu)$, we recall the generalization of the rejection method described in Chap. 2. Given $P(y)=C_{0} F(y) Q(y)$, $a \leqslant y \leqslant b$, where $P(y)$ and $Q(y)$ are probability densities, $0 \leqslant F(y) \leqslant 1$, and $\mathrm{C}_{0}>1$ is any constant. If $\mathrm{y}$ is sampled from the density function $\mathrm{Q}(\mathrm{y})$, retained as the sample value of $y$ with probability $F(y)$ [and rejected with probability $1-F(y)$ ], then the set of $y$ values retained was shown to have density $P(y)$.

To apply this to incoherent scattering, we write $\sigma_{\mathrm{t}}^{\mathrm{i}}(\mathrm{Z}, \alpha)$ and $\sigma_{\mathrm{t}}(\alpha)$ for the total incoherent and Klein-Nishina cross sections, respectively, and express the probability density for scattering into $(\mu, \mu+\mathrm{d} \mu)$ in the form

$$
\begin{aligned}
\mathrm{p}(\mu) & =\frac{\sigma^{\mathrm{i}}(\mathrm{Z}, \alpha, \mu)}{\sigma_{\mathrm{t}}^{\mathrm{i}}(\mathrm{Z}, \alpha)} \\
& =\frac{\mathrm{I}(\mathrm{Z}, \overline{\mathrm{v}}) \sigma_{\mathrm{t}}(\alpha)}{\sigma_{\mathrm{t}}^{\mathrm{i}}(\mathrm{Z}, \alpha)} \times \frac{\mathrm{I}(\mathrm{Z}, \mathrm{v})}{\mathrm{I}(\mathrm{Z}, \overline{\mathrm{v}})} \times \frac{\mathrm{K}(\alpha, \mu)}{\sigma_{\mathrm{t}}(\alpha)} \\
& =\mathrm{C}_{0} \mathrm{~F}(\mu) \mathrm{Q}(\mu)
\end{aligned}
$$

We therefore sample $\mathrm{K}(\alpha, \mu) / \sigma_{\mathrm{t}}(\alpha)$ for $\mu$, which is retained with probability $\mathrm{F}(\mu)=$ $\mathrm{I}[\mathrm{Z}, \mathrm{v}(\alpha, \mu)] / \mathrm{I}(\mathrm{Z}, \overline{\mathrm{v}}) \leqslant 1$.

Tables of the scattering factors $I(Z, v)$ are available in the literature. In the Los Alamos code MCP, 6 the complete tabulations of Cromer and Mann ${ }^{7,8}$ (and of Brown $^{9}$ for a few low $Z$ ) are used for all $Z \geqslant 2, v \leqslant 8$, and we can $\operatorname{set} I(Z, v) \equiv Z$ for $v>8$ without any noticeable impairment of accuracy. For $Z=1$ an exact formula is available. ${ }^{10}$

\section{COHERENT SCATTERING}

This type of scattering is important at low energies when the electron must be considered bound. The mass of a bound electron is effectively that of the entire atom, and a good assumption is that no energy loss occurs in this process. Only the angle of deflection is changed in the collision. Because all atomic electrons behave similarly, the radiation scattered by the individual bound electrons of a given atom will be coherent, i.e., capable of showing constructive or destructive interference. We can write the differential scattering cross section in the form

$$
\sigma^{\mathrm{C}}(\mathrm{Z}, \alpha, \mu) \mathrm{d} \mu=\mathrm{C}^{2}(\mathrm{Z}, \mathrm{v}) \mathrm{T}(\mu) \mathrm{d} \mu
$$

where $\mathrm{C}(\mathrm{Z}, \mathrm{v})$ is a scattering factor modifying the energy-independent Thomson cross section $T(\mu)=\pi r_{0}^{2}\left(1+\mu^{2}\right)$. The scattering factor represents the probability that the 
$\mathrm{Z}$ electrons of an atom take up the recoil momentum without absorbing any energy. The notation is the same as that given previously.

The effect of $\mathrm{C}^{2}(\mathrm{Z}, \mathrm{v}) / \mathrm{Z}^{2}$ is to decrease the Thomson cross section more extremely for backward scattering, high $E$, and low $Z$, which is opposite to the effect of $I(Z, v) / Z$ on the Klein-Nishina cross section described in the preceding section. For a given $Z$, $C(Z, v)$ decreases from $C(Z, 0)=Z$ to $C(Z, \infty)=0$. For example, $C(Z, v)$ is a rapidly decreasing function of $\mu$ as $\mu$ varies from +1 to -1 , and therefore the coherent cross section is peaked in the forward direction. At high energies of the incoming photon, coherent scattering is strongly forward and can be ignored. The parameter $\mathrm{v}=$ $\kappa \alpha(1-\mu)^{1 / 2}$ is identical to that used in the discussion of incoherent scattering. The qualitative features of $\mathrm{C}(\mathrm{Z}, \mathrm{v})$ are shown in Fig. 6.2.

Values of the scattering factor $C(Z, v)$ are documented in a number of references. For example, the Los Alamos code $\mathrm{MCP}^{6}$ uses tables of $\mathrm{C}(\mathrm{Z}, \mathrm{v})$ for $\mathrm{Z} \geqslant 1, \mathrm{v} \leqslant 6$, which were compiled from various sources ${ }^{11-13}$ with values listed for $v_{1}=0, \ldots$, $\mathrm{v}_{55}=6$. (For details, see Storm and Israel. ${ }^{14}$ ) For practical purposes, we can define $\mathrm{C}(\mathrm{Z}, \mathrm{v}) \equiv 0$ for $\mathrm{v}>6$.

To sample for the scattering angle in coherent scattering, we can again use the rejection technique used in the preceding section. However, it is convenient to follow a device used in the SORS photon code ${ }^{15}$ and reverse the roles of the coherent crosssection components. Defining $\mathrm{p}^{\mathrm{c}}(\mu)=\sigma^{\mathrm{c}}(\mathrm{Z}, \alpha, \mu) / \sigma_{\mathrm{t}}^{\mathrm{c}}(\mathrm{Z}, \alpha)$, the probability density function of $\mu$, where $\sigma_{\mathrm{t}}^{\mathrm{c}}$ is the total coherent cross section, we can transform to the

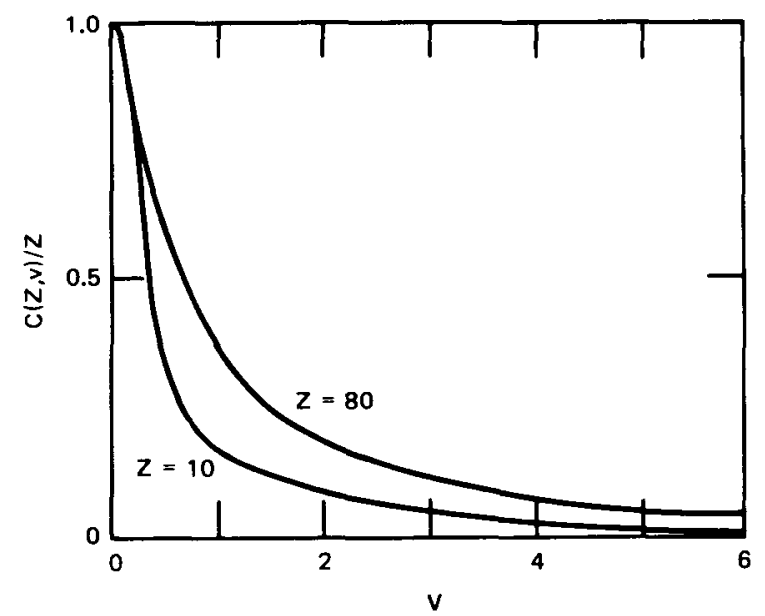

Fig. 6.2 Coherent scattering factor. 
variable $\mathrm{v}^{2}$ as follows:

$$
\mathrm{P}\left(\mathrm{v}^{2}\right) d \mathrm{v}^{2}=\mathrm{p}^{\mathrm{c}}(\mu)\left|\frac{\mathrm{d} \mu}{d \mathrm{v}^{2}}\right| d \mathrm{v}^{2}
$$

where $\mu$ is replaced by the variable $v^{2}=(\kappa \alpha)^{2}(1-\mu), 0 \leqslant v^{2} \leqslant \bar{v}^{2}$. Since $\mu=$ $1-\left[v^{2} /(\kappa \alpha)^{2}\right], d \mu / \mathrm{dv}^{2}=-1 /(\kappa \alpha)^{2}$, and we can write

$$
\begin{aligned}
\mathrm{P}\left(\mathrm{v}^{2}\right) \mathrm{dv^{2 }} & =\frac{2 \pi \mathrm{r}_{0}^{2} \mathrm{Z}^{2} \mathrm{~A}\left(\mathrm{Z}, \overline{\mathrm{v}}^{2}\right)}{(\kappa \alpha)^{2} \sigma_{\mathrm{t}}^{\mathrm{c}}(\mathrm{Z}, \alpha)}\left(\frac{1+\mu^{2}}{2}\right) \mathrm{Q}\left(\mathrm{v}^{2}\right) \mathrm{dv^{2 }} \\
& \equiv \mathrm{C}_{0} \mathrm{~F}\left(\mathrm{v}^{2}\right) \mathrm{Q}\left(\mathrm{v}^{2}\right) \mathrm{dv^{2 }}
\end{aligned}
$$

where

$$
\begin{gathered}
Q\left(v^{2}\right)=\frac{C^{2}(Z, v) Z^{-2}}{A\left(Z, \bar{v}^{2}\right)} \\
A\left(Z, v^{2}\right)=\int_{0}^{v^{2}} C^{2}(Z, v) Z^{-2} d v^{2}
\end{gathered}
$$

for arbitrary $\mathrm{v}^{2}$.

A random number $\xi$ on $[0,1]$ can therefore be used to tentatively assign $v^{2}$ with density $Q\left(v^{2}\right)$ by the relation $\xi=A\left(Z, v^{2}\right) / A\left(Z, \bar{v}^{2}\right), v^{2}$ being accepted with probability $F\left(v^{2}\right)=\left(1+\mu^{2}\right) / 2 \leqslant 1$, where $\mu$ is the above function of $v^{2}$. The required values of $A\left(Z, \bar{v}^{2}\right)$ and $v^{2}$ can be found by linear interpolation using tables of $A\left(Z, v_{i}^{2}\right)$ for $v_{1}^{2}=0, \ldots, v_{55}^{2}=36$ obtained by numerical integration and stored in the program. 6

\section{PHOTOELECTRIC EFFECT}

In this process the incident photon of energy $\mathrm{E}$ disappears, an orbital electron with kinetic energy $\mathrm{E}-\mathrm{e}$ is ejected from some (positively written) energy level $\mathrm{e} \leqslant \mathrm{E}$, and a second electron from an energy level $\mathrm{e}^{\prime}<\mathrm{e}$ makes the transition to the e-level vacancy. There are two possibilities.

1. A fluorescence photon of energy $E^{\prime}=e-e^{\prime}$ may be emitted. Then the photonenergy difference $E-E^{\prime}=(E-e)+e^{\prime}$ consists of the kinetic energy of the first ejected electron plus a residual excitation energy $e^{\prime}$ that is ultimately dissipated by further processes with additional fluorescence of still lower energy. In some computer programs with an energy cutoff in the neighborhood of $1 \mathrm{keV}$, this additional fluorescence is ignored, the energy $\mathrm{E}-\mathrm{E}^{\prime}$ is deposited locally, and the photon of energy $E^{\prime}$ is processed further if $E^{\prime}$ is greater than the energy cutoff. However, in the 
following we describe a treatment of the most important secondary fluorescence photons. All fluorescence photons are assumed to be emitted isotropically.

2. The electron transition $\mathrm{e}^{\prime} \rightarrow \mathrm{e}$ may not be accompanied by $\mathrm{E}^{\prime}=\mathrm{e}-\mathrm{e}^{\prime}$ fluorescence but instead by the ejection of an Auger electron resulting from internal conversion. In a Monte Carlo treatment of this case, the entire incident energy $\mathrm{E}$ is tallied as energy deposition, and the collision is terminal.

The energy levels e are called edge energies because the photoelectric cross section $\sigma(\mathrm{E})$, elsewhere decreasing continuously, shows a sharp discontinuity (edge) at each $\mathrm{E}=\mathrm{e}$. The cross section jumps from its lower limiting value $\sigma^{\prime}(\mathrm{e})$ to its value $\sigma(\mathrm{e})>$ $\sigma^{\prime}(\mathrm{e})$ as the photon energy E becomes sufficient to activate the e-level. The behavior at the $\mathrm{K}$ edge is shown in Fig. 6.3.

To illustrate a Monte Carlo treatment of the photoelectric effect, we shall describe the method ${ }^{16}$ that is presently incorporated in the Los Alamos code MCP. ${ }^{6}$ This method supersedes the original MCP scheme, which was patterned after that used in PHOTRAN ${ }^{17}$ and used the tabulated data of Marotta. ${ }^{18}$ The present method uses basic data from the tables of Storm and Israel. ${ }^{14}$ Fluorescence is not considered from shells other than $\mathrm{K}$ and $\mathrm{L}$. The probability of exciting other shells and the correresponding yields are both small and are maximal for high $Z$, for which the photoelectric cross section is enormous at the fluorescent energies. Therefore local absorption for such fluorescence is assumed. For $\mathrm{Z}<12$, a photoelectric event is regarded as terminal, the possible fluorescence energy being below $1 \mathrm{keV}$, the cutoff energy in MCP.

Given a photoelectric event at incident-photon energy $E$, the purpose of the fluorescence routine is to determine which (if either) of the two shells, $\mathrm{K}$ or L, loses an electron and the fluorescence photon energy emitted.

1. Edge Energies. The K-edge energies $\mathrm{E}_{\mathrm{K}}$ are taken from Table II of Ref. 14. The $L$-edge energy (for $Z \geqslant 31$ ) is regarded as the simple average $\bar{E}_{L}=\left(E_{L 1}+E_{L 2}+\right.$ $\left.E_{L_{3}}\right) / 3$, the individual $E_{L i}$ being taken from Table II of Ref. 14. The energies $E_{K}$ and $\overline{\mathrm{E}}_{\mathrm{L}}$, compared with the incident-photon energy $\mathrm{E}$, serve to determine the possibility of $\mathrm{K}$ or $\mathrm{L}$ events.

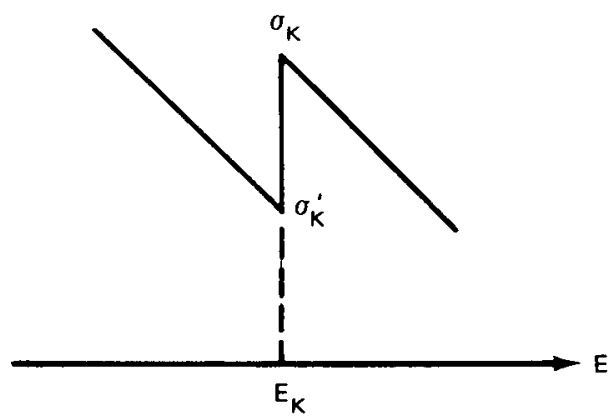

Fig. 6.3 Behavior of $\sigma(\mathrm{E})$ at the $\mathrm{K}$ edge. 
2. Fluorescence Energies F. L fluorescence is allowed only for $\mathrm{Z} \geqslant 31$, and the single fluorescence energy $F_{L}$ is taken as $\bar{L}_{123}$ in Table $V$ of Ref. 14. This is an average of all energy gaps from edges $\mathrm{Mj}, \mathrm{Nk}, \ldots$ to all $\mathrm{Li}$ edges, weighted by their relative intensities as given in Tables IV and VI of Ref. 14.

The single value $F_{K}$ given for $12 \leqslant Z \leqslant 19$ is the weighted average of the $\mathrm{K} \alpha_{1}(\mathrm{~L} 3 \rightarrow \mathrm{K})$ and $\mathrm{K} \alpha_{2}(\mathrm{~L} 2 \rightarrow \mathrm{K})$ lines, given as $\mathrm{K} \alpha$ in Table $\mathrm{V}$ of Ref. 14.

For $20 \leqslant Z \leqslant 94$, the individual fluorescence energies $\mathrm{F}_{\mathrm{K} \alpha_{1}}>\mathrm{F}_{\mathrm{K} \alpha_{2}}$ are taken from Ref. 14, Table III. These are pure lines resulting from the transitions indicated above.

For $20 \leqslant Z \leqslant 94$, the fluorescence energy $F_{K \beta_{1}^{\prime}}(M 2 \rightarrow K, M 3 \rightarrow K, M 4 \rightarrow K)$ given is the weighted average of the three energy differences, computed from Tables III and VI of Ref. 14.

For $37 \leqslant Z \leqslant 94$, the energy $\mathrm{F}_{\mathrm{K} \beta_{2}^{\prime}}(\mathrm{N} 2 \rightarrow \mathrm{K}, \mathrm{N} 3 \rightarrow \mathrm{K})$ is the corresponding weighted average of these two lines. Note that $\mathrm{F}_{\mathrm{L}}<\mathrm{F}_{\mathrm{K} \alpha_{2}}<\mathrm{F}_{\mathrm{K} \alpha_{1}}<\mathrm{F}_{\mathrm{K}_{\beta_{1}^{\prime}}}<\mathrm{F}_{\mathrm{K} \beta_{2}^{\prime}}$.

3. The Yields $\mathrm{Y}_{\mathrm{K}}$ and $\mathrm{Y}_{\mathrm{L}}$. The yield $\mathrm{Y}_{\mathrm{S}}$ for a shell $\mathrm{S}$ is the total probability of fluorescent emission accompanying electron transitions from outer shells to a vacancy (see item 6).

The yield $\mathrm{Y}_{\mathrm{K}}$, furnished by Israel and Storm, is an updated version of that in Ref. 19, Table VIII. For this we have no published reference; no yields are included in Ref. 14. These updated yields are in general accord with Ref. 20, Table II, and the yields for $Z>60$ are identical with those in Ref. 19 but are higher for $Z \leqslant 60$.

The data for $Y_{L}$ in Ref. 20 are very spotty, and the values now used from Ref. 19, Table VIII, do not appear to be a bad compromise between those of Refs. 18 and 20.

4. Relative Probabilities of $\mathrm{K}$ and $\mathrm{L}$ Events. For $12 \leqslant \mathrm{Z} \leqslant 30$, only $\mathrm{K}$ fluorescence is considered, and this can occur only for $E>E_{K}$. For such an incident energy $E$, it is assumed (see Ref. 14, page 569) that the probability of ejecting an electron from the $\mathrm{K}$ shell has the constant value $\left(\sigma_{\mathrm{K}}-\sigma_{\mathrm{K}}^{\prime}\right) / \sigma_{\mathrm{K}}=1-\rho_{\mathrm{K}}$, where $\rho_{\mathrm{K}}=\sigma_{\mathrm{K}}^{\prime} / \sigma_{\mathrm{K}}$ is the ratio of the photoelectric cross sections at the bottom and the top of the $\mathrm{K}$ edge, respectively (Fig. 6.3). Here and elsewhere, $\sigma^{\prime}$ and $\sigma$ are taken from Table I of Ref. 14. Thus, for a photoelectric collision at an energy $\mathrm{E} \geqslant \mathrm{E}_{\mathrm{K}}, 1-\rho_{\mathrm{K}}$ is the probability $\because$ a $\mathrm{K}$ ejection, and $\left(1-\rho_{\mathrm{K}}\right) \mathrm{Y}_{\mathrm{K}}$ is the probability of a contingent $\mathrm{K}$ fluorescence. (Observe that $1-\rho_{\mathrm{K}}$ is the entry $\sigma_{\mathrm{K}}$ (photo) $/ \sigma$ (photo) given in Table VIII of Ref. 14.)

In the range $31 \leqslant Z \leqslant 94$, both $L$ and $K$ ejections are considered, and, in order to follow the scheme of Ref. 18 used previously with no change in code, we require three numbers $\Phi_{\mathrm{K}}, \Phi_{\mathrm{L}}, \Phi_{0}$, which will give the relative probabilities of K-, L-, or outer-shell events for $E \geqslant E_{K}$ and are such that $\Phi_{L}, \Phi_{0}$ also define the chances of $L$ - or outershell events for $\overline{\mathrm{E}}_{\mathrm{L}} \leqslant \mathrm{E} \leqslant \mathrm{E}_{\mathrm{K}}$.

The basic assumption is that the relative contribution of any edge to the total photoelectric cross section at that edge is $\left(\sigma-\sigma^{\prime}\right) / \sigma$ and that this relative contribution remains constant at higher energies. If we define $\rho_{\mathrm{i}}=\sigma_{\mathrm{i}}^{\prime} / \sigma_{\mathrm{i}}, \mathrm{i}=1,2,3$, (Fig. 6.4), it follows that $\rho_{\mathrm{L}}=\rho_{3} \rho_{2} \rho_{1}$ and $1-\rho_{\mathrm{L}}$ are the probabilities of outer-shell events (and 
hence no fluorescence) and L-shell events at the $\mathrm{L}_{1}$ edge, the latter being given in Table VIII, column 2, of Ref. 14 with some minor discrepancies.

Similarly, at the $\mathrm{K}$ edge, with $\rho_{\mathrm{K}}=\sigma_{\mathrm{K}}^{\prime} / \sigma_{\mathrm{K}}$, we find that $\rho_{\mathrm{L}} \sigma_{\mathrm{K}}^{\prime} / \sigma_{\mathrm{K}}=\rho_{\mathrm{L}} \rho_{\mathrm{K}}$, $\left(1-\rho_{\mathrm{L}}\right) \sigma_{\mathrm{K}}^{\prime} / \sigma_{\mathrm{K}}=\left(1-\rho_{\mathrm{L}}\right) \rho_{\mathrm{K}}$, and $\left(\sigma_{\mathrm{K}}-\sigma_{\mathrm{K}}^{\prime}\right) / \sigma_{\mathrm{K}}=1-\rho_{\mathrm{K}}$ are the probabilities of outer-, L-, and K-shell events at the $\mathrm{K}$ edge. For convenience, we take the proportional numbers

$$
\begin{aligned}
& \Phi_{0}=\rho_{\mathrm{L}} \\
& \Phi_{\mathrm{L}}=1-\rho_{\mathrm{L}} \\
& \Phi_{\mathrm{K}}=\rho_{\mathrm{K}}^{-1}-1
\end{aligned}
$$

as giving the corresponding relative probabilities, and these satisfy the required conditions for all energies $E$, as stipulated above. Moreover, given the numbers $0, \Phi_{L} Y_{L}$, $\Phi_{K} Y_{K}$ if, for $E_{L} \leqslant E \leqslant E_{K}$, we divide each of the first two by $\Phi_{0}+\Phi_{L}=1$ and, for $E \geqslant E_{K}$, we divide each of the three by $\Phi_{0}+\Phi_{L}+\Phi_{K}=\rho_{K}^{-1}$, then in each case we obtain the probability of outer, $\mathrm{L}$, or $\mathrm{K}$ fluorescence, as the case may be.

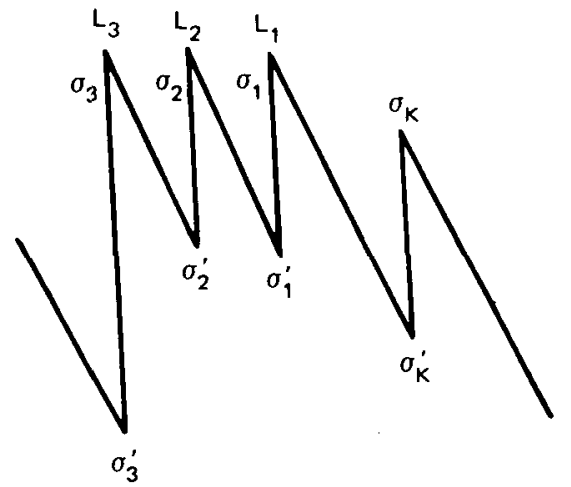

Fig. 6.4 Schematic of $K$ and $L$ edges.

5. Relative Intensities of $\mathrm{K}$ Fluorescence. The preceding discussion suffices to give the total chance $\Phi_{K} Y_{K} /\left(\Phi_{0}+\Phi_{L}+\Phi_{K}\right)$ of $K$ fluorescence, assuming a $K$ event with $E \geqslant E_{K}$. However, for $20 \leqslant Z \leqslant 36$, such fluorescence was assumed to consist of the lines $K \alpha_{1}, K \alpha_{2}$, and $K \beta_{1}^{\prime}$, and, for $37 \leqslant Z \leqslant 94$, of the additional line $K \beta_{2}^{\prime}$. The corresponding probabilities $\mathrm{p}_{1}, \mathrm{p}_{2}, \mathrm{p}_{3}$ and $\mathrm{p}_{1}, \mathrm{p}_{2}, \mathrm{p}_{3}, \mathrm{p}_{4}$ of the component lines for the two ranges of $Z$ are computed from the table of relative intensities given in Ref. 14, Table VI, based on the calculations of Schofield.

Let us illustrate the foregoing remarks with a few examples. Decisions regarding fluorescence are based on comparison of $E$ with $\bar{E}_{L}$ and $E_{K}$. If $E<\bar{E}_{L}$, no fluores- 
cence is allowed. For $\overline{\mathrm{E}}_{\mathrm{L}} \leqslant \mathrm{E} \leqslant \mathrm{E}_{\mathrm{K}}, \Phi_{\mathrm{L}} \mathrm{Y}_{\mathrm{L}} /\left(\Phi_{0}+\Phi_{\mathrm{L}}\right)=\left(1-\rho_{\mathrm{L}}\right) \mathrm{Y}_{\mathrm{L}}$ is the chance of obtaining the fluorescence energy $F_{L}$. Similarly, for $E \geqslant E_{K}$, the chance of $F_{L}$ fluorescence is $\Phi_{\mathrm{L}} \mathrm{Y}_{\mathrm{L}} /\left(\Phi_{0}+\Phi_{\mathrm{L}}+\Phi_{\mathrm{K}}\right)=\mathrm{Y}_{\mathrm{L}}\left(1-\rho_{\mathrm{L}}\right) \rho_{\mathrm{K}}$, and the chance of exciting one of the $\mathrm{K}$ lines, say, $\mathrm{F}_{\mathrm{K} \alpha_{2}}$, is $\Phi_{\mathrm{K}} \mathrm{Y}_{\mathrm{K}} \mathrm{p}_{2} /\left(\Phi_{0}+\Phi_{\mathrm{L}}+\Phi_{\mathrm{K}}\right)=\left(\rho_{\mathrm{K}}^{-1}-1\right) \mathrm{Y}_{\mathrm{K}} \mathrm{p}_{2} \rho_{\mathrm{K}}=$ $\left(1-\rho_{\mathrm{K}}\right) \mathrm{Y}_{\mathrm{K}} \mathrm{p}_{2}$.

6. Secondary Fluorescence. The type of fluorescence considered in the preceding remarks is primary, in the sense that it arises from the transition of an electron from an outer shell to a shell in which a vacancy has been created by ejection of an electron from that shell by the initial incident photon. Thus we allow for $L$ fluorescence following an $\mathrm{L}$ ejection and for $\mathrm{K}$ fluorescence following a $\mathrm{K}$ ejection.

In the following remarks, we attempt to show roughly how secondary L fluorescence may be of the same order of importance as primary L fluorescence and then to describe how it may be accounted for in a necessarily approximate fashion.

Let us assume that we have a photoelectric event with an element of $Z \geqslant 31$ at an incident energy $E \geqslant E_{K}$. Then the probability of an $L$ ejection is $\Phi_{L} / \Sigma$, where $\Sigma=\Phi_{0}+\Phi_{L}+\Phi_{K}$ and, as already stated, the overall probability of primary L fluorescence is given by

$$
\mathrm{P}^{\prime}=\frac{\Phi_{L}}{\Sigma} \mathrm{Y}_{\mathrm{L}}
$$

On the other hand, there is a probability $\Phi_{K} / \Sigma$ of a $\mathrm{K}$ ejection. In this event the $\mathrm{K}$ vacancy may be filled by an $\mathrm{L} 3 \rightarrow \mathrm{K}$ or $\mathrm{L} 2 \rightarrow \mathrm{K}$ transition, say with probabilities $\mathrm{P}_{\mathrm{L} 3 \mathrm{~K}}, \mathrm{P}_{\mathrm{L} 2 \mathrm{~K}}$, respectively, thus creating a vacancy in the $\mathrm{L} 3$ - or $\mathrm{L} 2$-subshell and at the same time producing a $K \alpha_{1}$ or $K \alpha_{2}$ photon. If we denote by $\mathrm{Q}_{\mathrm{L} 3 \mathrm{~K}}, \mathrm{Q}_{\mathrm{L} 2 \mathrm{~K}}$ the chances of the latter photon escaping the atom (as fluorescence), then we obtain as part of the $\mathrm{K}$ yield the quantity

$$
P_{L 3 K} Q_{L 3 K}+P_{L 2 K} Q_{L 2 K}=Y_{K} p_{1}+Y_{K} p_{2}
$$

the right-hand side being written in our previous notation.

However, regarding the L3- and L2-subshells as separate entities, we may expect secondary L3 or L2 fluorescence with yield probabilities $\mathrm{Y}_{\mathrm{L} 3}$ and $\mathrm{Y}_{\mathrm{L} 2}$ due to the now-existing vacancies created by the $L \rightarrow K$ transition. Hence the probability of this secondary L fluorescence is given by

$$
\mathrm{P}^{\prime \prime}=\frac{\Phi_{\mathrm{K}}}{\Sigma}\left(\mathrm{P}_{\mathrm{L} 3 \mathrm{~K}} \mathrm{Y}_{\mathrm{L} 3}+\mathrm{P}_{\mathrm{L} 2 \mathrm{~K}} \mathrm{Y}_{\mathrm{L} 2}\right)
$$

Apparently none of the probabilities in parenthesis are known. Making the assumption

$$
Y_{L 3}=Y_{L 2}=Y_{L 1}=\frac{Y_{L}}{3}
$$


we find the expression in Eq. 6.14 for $\mathrm{P}^{\prime \prime}$ becomes

$$
\mathrm{P}^{\prime \prime}=\frac{\Phi_{\mathrm{K}}}{\Sigma} \frac{\left(\mathrm{P}_{\mathrm{L} 3 \mathrm{~K}}+\mathrm{P}_{\mathrm{L} 2 \mathrm{~K}}\right) \mathrm{Y}_{\mathrm{L}}}{3}
$$

But from Eqs. 6.13 and 6.16 , we see that for the secondary L fluorescence

$$
\mathrm{P}^{\prime \prime} \geqslant \frac{\Phi_{\mathrm{K}}}{\Sigma} \frac{\left(\mathrm{Y}_{\mathrm{K}} \mathrm{p}_{1}+\mathrm{Y}_{\mathrm{K}} \mathrm{p}_{2}\right) \mathrm{Y}_{\mathrm{L}}}{3} \equiv \mathrm{P}^{*}
$$

and even $\mathrm{P}^{*}$ may exceed the primary probability $\mathrm{P}^{\prime}$. For example, using the basic data from Storm and Israel cited above for $Z=79$, we find that $P^{\prime}=0.058<P^{*} \cong 0.075 \leqslant$ $\mathrm{P}^{\prime \prime}$. Thus it would appear that secondary $\mathrm{L}$ fluorescence should be included for the sake of consistency and accuracy.

Guided by Eq. 6.17 we can make the following approximation with regard to secondary $\mathrm{L}$ fluorescence, admittedly oversimplified but perhaps better than ignoring it. In the event of a photoelectric event with an atom of $Z \geqslant 31$ at an energy $E \geqslant E_{K}$, which is followed by $\mathrm{K} \alpha_{1}$ or $\mathrm{K} \alpha_{2}$ fluorescence, assume that secondary L3 or L2 fluorescence is emitted in each case with probability $Y_{L} / 3$ and fluorescent energy $F_{L}$.

\section{PAIR PRODUCTION}

This reaction becomes increasingly important for energies greater than $1.022 \mathrm{MeV}$. In the field of charged particles, mainly in the nuclear field but to some extent also in the field of an electron, the photon is completely absorbed, and a positron-electron pair appears with a total energy equal to that of the photon. Some of the energy goes into the kinetic energy of the electron and positron, but $1.022 \mathrm{MeV}$ is required to create the electron-positron pair since the rest energy of each of these particles is $\mathrm{m}_{0} \mathrm{c}^{2}=0.511 \mathrm{MeV}$. A pair is produced by absorption of the photon only in the presence of a charged particle since the charged particle is required for conservation of momentum.

The positron created in pair production combines with an electron in an interaction in which the rest masses of the electron and positron disappear and two quanta of energy totaling $2 \mathrm{~m}_{0} \mathrm{c}^{2}$ appear. If the positron is assumed to be essentially at rest at annihilation so that the momentum of the center of mass of the resulting two-body system is zero in the laboratory system, then the two quanta will appear in opposite directions, each having an energy equivalent to the rest energy of an electron, or 0.511 $\mathrm{MeV}$.

In many Monte Carlo codes $^{6}$ treating gamma rays of energy $E>1.022 \mathrm{MeV}$, when pair production occurs, an amount of energy equal to $\mathrm{E}-1.022 \mathrm{MeV}$ is deposited locally, and two gammas, each of energy $0.511 \mathrm{MeV}$, appear in opposite directions and are transported farther. The angular distribution of the annihilation quanta is as- 
sumed to be isotropic. In such a simplified treatment, the bremsstrahlung associated with the change in velocity of the electron and positron is ignored, just as it is in connection with the recoil electron in Compton scattering.

For many photon-transport problems, the assumptions made above are adequate. However, experiment and theory now indicate that the omission of a suitable treatment of bremsstrahlung from secondary electrons can lead to serious errors in many cases. In particular, this is indicated for high-energy photons impinging on a high- $Z$ material. A thorough discussion of electron transport and the accompanying bremsstrahlung is given in an article by Berger and Seltzer ${ }^{21}$ and in the dissertation by Thompson. ${ }^{22}$ The theoretical considerations by Thompson are presently being incorporated into general three-dimensional transport codes at Los Alamos.

\section{COMBINED NEUTRON AND PHOTON TRANSPORT}

Given the existence of Monte Carlo codes that transport neutrons and photons separately, our next logical step is to combine the two types into one code. Then we can process the gamma rays produced by the collisions of the neutrons in the medium as well as the neutrons themselves. Storage of gamma-production cross sections in the code permits the linking of the neutron- and gamma-transport codes.

Let us describe in more detail how the Los Alamos code $\mathrm{MCNG}^{23}$ operates. The MCNG code is made up by combining the neutron code MCN and the gamma code MCG. Gamma-production cross sections are provided on a cross-section tape along with the required neutron and gamma cross sections. Cross-section data for the materials in the problem are drawn from this tape. Neutrons are processed by $\mathrm{MCN}$, and at each collision gammas are created and stored on a tape. To reduce the variance in estimating the heating in a region (the charged-particle energy is assumed to be deposited locally as heat), we compute the expected charged-particle heating per collision, i.e., we calculate the expected gamma energy $\Gamma^{\prime}$ and the expected neutron energy $E^{\prime}$ leaving the collision. Using these expected quantities and $\bar{Q}$, the average $Q$ value for the incoming neutron energy $\mathrm{E}$, we obtain the charged-particle heating $\mathrm{H}$ per collision from the relation

$$
\mathrm{H}=\mathrm{E}+\overline{\mathrm{Q}}-\mathrm{E}^{\prime}-\Gamma^{\prime}
$$

Further heating of the medium may occur from energy deposited from the transport of the gammas. After a prescribed number of neutrons are processed, the gammas created are transported. The code cycles between the neutron code MCN and the gamma code MCG until the required accuracy is attained in the heating numbers or other quantities of interest in the problem (all results are normed per starting neutron). Because of the large number of low-weight gammas created by neutron collisions, Russian roulette is played for particles with weight below a lower weight bound, the result being to form fewer particles of larger weight. Heating in small regions can be obtained with a track-length estimator. 


\section{REFERENCES}

1. E. D. Cashwell and C. J. Everett, A Practical Manual on the Monte Carlo Method for Random Walk Problems, Pergamon Press, Inc., New York, 1959.

2. Graphs of the Compton Energy-Angle Relationship and the Klein-Nishina Formula from 10 keV to $500 \mathrm{MeV}$, National Bureau of Standards, Circular No. 542, Superintendent of Documents, U.S. Government Printing Office, Washington, 1953.

3. H. Kahn, Applications of Monte Carlo, USAEC Report AECU-3259, Rand Corporation, 1954.

4. C. J. Everett and E. D. Cashwell, Approximation for the Inverse of the Klein-Nishina Probability Distribution, USAEC Report LA-3839, Los Alamos Scientific Laboratory, 1970.

5. C. J. Everett, E. D. Cashwell, and G. D. Turner, A New Method of Sampling the KleinNishina Probability Distributions for All Incident Photon Energies Above $1 \mathrm{keV}$, USAEC Report LA-4663, Los Alamos Scientific Laboratory, 1971.

6. E. D. Cashwell, J. R. Neergaard, C. J. Everett, R. G. Schrandt, W. M. Taylor and G. D. Turner, Monte Carlo Photon Codes: MCG and MCP, USAEC Report LA-5157-MS, Los Alamos Scientific Laboratory, 1973.

7. D. T. Cromer and J. B. Mann, Compton Scattering Factors for Spherically Free Atoms, $J$. Chem. Phys., 47:1892-1893 (1967).

8. D. T. Cromer, Compton Scattering Factors for Aspherical Frec Atoms, J. Chem. Phys., 50:4857-4859 (1969).

9. R. T. Brown, Edgerton, Germeshausen \& Grier, Inc., Technical Report L-958, 1969, and unpublished data.

10. G.W. Grodstein, X-Ray Attenuation Coefficients from $10 \mathrm{keV}$ to $100 \mathrm{MeV}$, National Bureau of Standards, Circular No. 583, Superintendent of Documents, U.S. Government Printing Office, Washington, 1957.

11. P. A. Doyle and P. S. Turner, Relativistic Hartrec-Fock X-Ray Electron Scattering Factors, Acta Cryst., 24:390-397 (1968).

12. D. T. Cromer and J. T. Waber, Atomic Scattering Factors for X-Rays, in International Tables for X-Ray Crystallography, Vol. 4, pp. 71-147, Kynoch Press, Birmingham, England, 1974.

13. H. P. Hanson, F. Herman, J. D. Lea, and S. Skillman, HFS Atomic Scattering Factors, Acta Cryst., 17:1040-1044 (1964).

14. E. Storm and H. I. Israel, Photon Cross Sections from $1 \mathrm{keV}$ to $100 \mathrm{MeV}$ for Elements $\mathrm{Z}=1$ to $\mathrm{Z}=100$, At. Data Nucl. Data Tables, 7:565-681 (1970).

15. J.W. Kimlinger, E. F. Plechaty, and J. R. Terrall, SORS Monte Carlo Photon-Transport Code for the CDC 6600, USAEC Report UCRL-50358, University of California Lawrence Radiation Laboratory, 1967.

16. C. J. Everett and E. D. Cashwell, MCP Code Fluorescence-Routine Revision, USAEC Report LA-5240-MS, Los Alamos Scientific Laboratory, 1973.

17. C. D. Zerby, J. Agresta, et al., PHOTRAN, A General Purpose Photon Transport Program in Complex Geometry, Technical Report AFWL-TR-65171 (Vols. I-IV), Union Carbide Corporation, Rescarch Institute, 1966-1968.

18. C. R. Marotta, Updated Master Library Tape for PHOTRAN, Technical Report AFWLTR-67-11, Union Carbide Corporation, Research Institute, 1967.

19. E. Storm and H. I. Israel, Photon Cross Sections from 0.001 to $100 \mathrm{MeV}$ for Elements 1 through 100, USAEC Report LA-3753, Los Alamos Scientific Laboratory, 1967.

20. R. W. Fink et al., A tomic Fluorescence Yiclds, USAEC Report UCRL-14327, University of California Lawrence Radiation Laboratory, 1965.

21. M. J. Berger and S. M. Seltzer, Bremsstrahlung and Photoneutrons from Thick Tungsten and Tantalum Targets, Phys. Rev., C, 2 (2):621-631 (August 1970). 
22. W. L. Thompson, Gamma-Ray and Electron Transport by Monte Carlo, Ph.D. Dissertation, University of Virginia, Charlottesville, Va., February 1974.

23. E. D. Cashwell, C. J. Everett, C. A. Forest, R. G. Schrandt, W. M. Taylor, and G. D. Turner, MCNG: A Monte Carlo Neutron-Gamma Transport Code, Los Alamos Scientific Laboratory, report in preparation. 


\section{INTRODUCTION}

The emphasis in this chapter is on the literature of the last few years; very few references predate 1964 . The references are described briefly, and we focus on those we consider to be of general interest. This requires a judgment decision, and we apologize for any references that have been omitted or have not been described properly. The more recent and easily accessible literature has been given priority; so the work of an original contributor to a subject is sometimes omitted or only mentioned briefly in the interest of aiding the reader in his search for information.

The references cited are listed in general categories at the end of this chapter to help in the isolation of subjects of interest.

\section{BOOKS ON MONTE CARLO AND PROBABILITY THEORY}

A few books are available on the use of the Monte Carlo method to simulate particle transport. The Russian book ${ }^{1}$ edited by Shreider and translated by Tee is a useful reference for the engineer. It is directed toward applications, but enough mathematics is included to provide a background. The general principles of Monte Carlo are introduced in the first chapter and include a brief discussion of estimation and error analysis, the random walk, generation of random numbers, simulation of a Markov process, the computation of eigenvalues and eigenfunctions, and machine considerations. The evaluation of definite integrals is discussed in the second chapter. 
The application of the Monte Carlo method to neutron physics is the subject of the third chapter, which includes many useful comments on practical problems that arise in the simulation of particle transport. The next two chapters are concerned with applications of Monte Carlo outside the area of particle transport. The last two chapters discuss the generation of uniformly distributed random variables with computers and the transformation of random variables.

The book by Cashwell and Everett ${ }^{2}$ can serve as a handbook for the practitioner. It contains numerous relations that are required in programming a Monte Carlo code. The book is directed toward particle-transport problems and displays pertinent equations and flow diagrams for their simulation on a computer.

Spanier and Gelbard's book ${ }^{3}$ applies the Monte Carlo method to neutron-transport problems. A mathematical framework for the Monte Carlo calculation is developed in the first three chapters. The last three chapters are devoted to engineering applications, with emphasis on the superposition principle to solve reactor lattice problems.

The book by Hammersley and Handscomb 4 is another useful reference on Monte Carlo. Although not devoted exclusively to particle transport, it does provide another excellent reference and includes some pertinent material not covered in the other three books.

The reader may also find useful reference material in books that are not directly concerned with the simulation of particle transport. We include as possibilities the books on probability theory by Parzen ${ }^{5}$ and by Loeve ${ }^{6}$ and the book by Jansson ${ }^{7}$ on the machine gemeration of random numbers.

SAMPLING AND SCORING TECHNIQUES

The generation of random numbers on the unit interval and the techniques for using such numbers to sample from density functions are prerequisites for all Monte Carlo calculations. The book by Jansson, ${ }^{7}$ the article by Hull and Dobell, ${ }^{8}$ and the report by Lehmer ${ }^{9}$ provide helpful references for the machine generation of random numbers on the unit interval. The four books on Monte Carlo previously mentioned ${ }^{1-4}$ also discuss the generation of random numbers and display examples of the use of these numbers to sample density functions. There are a number of reports ${ }^{10-13}$ in which tests for randomness are discussed and applied for various random-number generators. Other references ${ }^{14-19}$ discuss sampling from a variety of commonly encountered density functions.

There is a considerable body of theory devoted to so-called quasirandom numbers. Whereas a sequence of pseudorandom numbers is intended for use in a wide variety of applications, a quasirandom number sequence is designed for a specific application and does not necessarily satisfy the statistical tests imposed on pseudorandom numbers. ${ }^{4}$ 
Foderaro $^{20}$ and Woolson ${ }^{21}$ consider decidedly nonrandom numbers on the unit interval to bias and hopefully improve the Monte Carlo transport calculation. They claim to achieve more uniform sampling on regions of equal importance, but it is not yet clear whether this use of nonrandom numbers has general advantages over the more common inportance sampling techniques used in particle-transport calculations.

The results of a Monte Carlo particle-transport calculation are subject to at least three sources of error: (1) inadequacies of physical models, (2) uncertainties in the basic cross-section data, and (3) the statistical nature of the Monte Carlo calculation itself. For most calculations the statistical error will usually approach zero as the number of samples approaches infinity. However, we cannot simulate an infinite number of samples, and thus we must be able to evaluate the error in the calculation. This introduces a practical problem since we cannot estimate the statistical uncertainty precisely unless we know the exact answer. Therefore we often quote the uncertainty to be within some confidence limit; i.e., the true answer lies within certain bounds with some probability. The error bounds are evaluated under certain assumptions that usually involve normality. References on Monte Carlo theory invariable have at least a brief discussion on the evaluation of statistical fluctuations. Burrows and MacMillan 22 also discuss a test of normality that is easy to apply.

Statistical errors are frequently estimated as proportional to the square root of the population variance. In particle-transport calculations this variance may change dramatically with the method used to score quantities of interest, and hence selection of the proper estimator is an important consideration. Popular estimators include the collision estimator, the path-length estimator, the last-event estimator, and the point-detector estimator. A discussion of various scoring techniques is presented in Refs. 23 to 29 as well as in the books on Monte Carlo. ${ }^{1-4}$ The point-detector estimator is a special type used to obtain information at a spatial point. Various point-detector estimators and a discussion of their variances are presented in Refs. 30 to 32 .

Correlated sampling is often effective in estimating the change in a quantity resulting from a small perturbation in the system. This technique enables the evaluation of small quantities that would otherwise be masked by the statistical errors of uncorrelated calculations. A useful discussion of correlated sampling and the evaluation of the resulting errors are given in the book by Spanier and Gelbard. ${ }^{3}$ Additional material is available in Refs. 33 and 34.

An alternate method of evaluating the effect of small perturbations is to compute derivatives of differences during the random walk. The article by Miller and Miley 52 on the computation of Doppler coefficients and the article by Takahashi ${ }^{35}$ on the geometrical perturbation of a pulsed reactor provide an introduction to the subject.

A careful evaluation of various sampling schemes is difficult since the theoretical variances cannot usually be computed exactly but must also be estimated by Monte Carlo. Amster ${ }^{36}$ and Spanier ${ }^{38}$ have considered some test cases where the theoretical variance can be either evaluated analytically or obtained by a relatively simple numerical calculation. 


\section{BIASING METHODS}

Importance sampling and biasing methods have received attention ever since Monte Carlo was first used for particle-transport calculations. A clear incentive has been provided by the fact that a zero-variance scheme exists for a wide class of transport problems. The construction of this optimal sampling scheme requires the solution of an equation that is typically equivalent in difficulty to solving the original equation, so the zero-variance scheme is not realized in practical problems.

For sampling schemes that are not optimal, it is often difficult to demonstrate that the efficiency of a biased Monte Carlo calculation is better than that of an analog sampling scheme, i.e., a straightforward modeling of the particle birth-death process. Here the efficiency factor of the Monte Carlo calculation of a functional is customarily defined to be inversely proportional to the product of the sample variance and the average computation time required per sample. A sampling scheme that increases the efficiency of some calculations may be disastrous in others, particularly if improperly used. As a rule of thumb, one must use caution when applying sampling schemes with the property that the particle weight may change many times in the course of a history. This is particularly true if the particle weight at any given phase-space point can become much larger than the average weight at that point. Ironically, schemes patterned after the theoretical zero-variance scheme tend to fall in this caution category. In the following paragraphs we mention a number of biasing methods and cite references that discuss these further.

Biasing the particle source is a technique that is simple and relatively safe to use in Monte Carlo calculations, and computer programs are usually written to allow for some source-biasing options. Source biasing is discussed in a number of places, e.g., Refs. 2, 3, and 39.

The analog density function for selecting collision points is sometimes normalized so that leakage out of the system does not occur, and the particle weight is appropriately reduced to account for this after each flight path. However, this method is not necessarily beneficial, because of the time wasted in computing the required nonleakage probability for each flight path and because many low-weight particles result. The interested reader is referred to Ref. 40 .

The exponential transform is a technique widely used to accelerate deep-penetration calculations. In our experience, we prefer to use cell importances rather than the exponential transform whenever possible, but the exponential transform does enjoy considerable use in the Monte Carlo community. It has some weight-stability properties that make it relatively safe to use. The interested reader is referred to Refs. $41-43$ and 53 .

The biasing of the energy and scattering angle at scattering events has become a popular subject during the last few years. ${ }^{44-47}$ The approach can yield significant gains; but this is also an area where caution should be exercised, and such biasing should only be used when the pitfalls are well understood. 
A problem in using importance sampling arises in choosing near-optimal biasing parameters. An approximate solution of the adjoint equation can be used to estimate these parameters for the random walk. ${ }^{48,104}$ Conversely, approximate calculations of the flux have been used to bias Monte Carlo simulations of adjoint equations. ${ }^{142}$ Some efforts have also been made to automate the computation of importance sampling. functions ${ }^{49,50}$ to relieve the user of such worries. One may also visualize a learning process in which the Monte Carlo code is written to improve itself as information is learned in the calculation. ${ }^{37,51}$

The maze of references on biasing techniques should not lead the uninitiated astray. Many Monte Carlo transport calculations can be completed with a reasonable amount of computational effort and yet use little or no biasing in the simulation. In fact, the Monte Carlo novice should try to use an analog simulation whenever practical, and he should apply biasing methods with caution. Most problems can be solved with simple source biasing along with the use of importances in regions for playing Russian roulette and splitting. We turn now to references on the mechanics of simulating neutron and photon transport.

\section{NEUTRON AND PHOTON TRANSPORT}

The mathematics for neutron-transport simulation is developed in the previously referenced books. ${ }^{1-4}$ Additional information regarding the treatment of the neutron interactions and related discussions are given in Refs. 53 to 61 and 105 to 108 . This is a broad subject area, and we will make no effort here to delineate further the various subtopics covered in these references.

Computer codes for simulating neutron transport tend to become obsolete in a few years, and, in addition, reviewers find it difficult to adequately evaluate specific code strengths and weaknesses. Hence individual computer programs will neither be discussed in detail nor recommended. References 62 to 79 describe a number of Monte Carlo codes for neutron transport as an introduction to the possibilities. This list undoubtedly does not include all the important codes, and therefore we recommend that potential Monte Carlo code users consult a code library, such as the Radiation Shielding Information Center (RSIC) at Oak Ridge National Labratory or the Argonne Code Center at Argonne National Laboratory for a more complete and up-to-date listing of major codes and their capabilities.

References 80 to 102 include discussions of numerous neutron-transport calculations that have been performed with Monte Carlo and contain other pertinent articles relating to the theory of Monte Carlo and techniques for applying it to neutron transport. These references are for the most part taken from the literature of the past few years. An excellent review of the older Monte Carlo literature is given by Kraft and Wensrich ${ }^{103}$ for the period from 1949 to 1963.

Many neutron-transport calculations pertain to multiplying systems. Special considerations in the use of Monte Carlo to compute criticality are given in Refs. 1 and 
4 as well as in numerous other places, including Refs. 54 and 105 to 110 . The specific problem of source convergence is discussed in Refs. 54 and 109 to 111 . Special problems concerned with error limits in source iteration calculations are considered in Refs. 112 and 113. We also include a number of other pertinent articles regarding criticality calculations with Monte Carlo. 14-124

Monte Carlo tends to have advantages over other numerical methods for computing the multiplication factor of small, fast critical assemblies. Mihalczo ${ }^{114}$ demonstrates that for some rather simple reflected systems, Monte Carlo criticality calculations can be faster than discrete-ordinates methods.

We turn now from neutron-transport references to photon-transport references. The simulation of photons corresponds closely to a simulation of neutrons, except that the physical models of the interactions differ. Cashwell and Everett ${ }^{2}$ include a consideration of important scattering mechanisms and techniques to sample them. In a later report by Cashwell et al.125 the simulation of the more sophisticated models necessary to treat low-energy (down to $1 \mathrm{keV}$ ) photon transport is discussed. Several additional articles applicable to photon transport appear in Refs. 126 to 136.

For problems where the $(n, \gamma)$ reaction and the subsequent gamma-ray transport are important, it is sometimes convenient to link the neutron- and photon-transport calculations. Two computer codes that simulate the neutron and photon transport are described in Refs. 77 and 78.

We also include here a number of specific applications of neutron- and photon-transport calculations with Monte Carlo. The first is its use in particle shielding. A good introduction to special problems in the use of Monte Carlo in solving shielding problems is presented in Refs. 137 and 138. Additional information on shielding calculations with Monte Carlo is given in Refs. 44 and 139 to 141 .

Another application of Monte Carlo in neutron and photon transport, which has become popular in the last few years, is the solution of equations that are adjoint to the transport equation. Certain classes of problems may be solved more efficiently via the adjoint approach, and, in addition, these solutions can be used to bias forward sampling methods. A variety of techniques for sampling from equations that are adjoint to the transport equation are given in Refs. 142 to 145. The interested reader will also find Refs. 146 to 154 pertinent to this general subject area.

Present-day computers are attaining such computational speeds that nonlinear radiative transport problems can sometimes be solved with an acceptable amount of computer time. The interested reader is referred to Refs. 155 to 159 . This is an active area of investigation, but many problems must still await solution until faster computers are available.

We turn now from specific applications of neutron and photon transport to a brief discussion of nuclear data. The ability to perform sophisticated particle-transport calculations is of questionable value unless reliable basic nuclear data are also available. Significant progress has been made in this direction during the past few years, and evaluated nuclear data files are now readily accessible. This relieves the user of some anxieties about cross sections, but he should always remember that reliable output depends upon good cross-section input. The user is also sometimes overwhelmed ${ }^{\mathbf{1 6 0}}$ 
by the large volume of cross-section data available from nuclear data files, and this can be nearly as frustrating as a complete lack of data. Levitt ${ }^{161}$ alleviates this problem by using a probability-table method to describe cross sections in the unresolved resonance region with a minimum amount of data. Cullen ${ }^{162}$ has extended the probability-table method to multigroup calculations.

The Evaluated Nuclear Data File, ENDF, maintained by the National Neutron Cross-Section Center at Brookhaven National Laboratory has become the most widely used source for neutron and photon cross sections. The interested reader may obtain introductory information on this library from Ref. 163 for neutron cross sections and from Ref. 164 for photon cross sections. A variety of cross-section processor codes are available to manipulate the data and perform multigroup averaging. Codes ${ }^{165-166}$ have also been written to utilize the ENDF data and obtain neutron-energy deposition KERMA factors.

A cross-section compilation by Howerton et al. ${ }^{167}$ also enjoys considerable use, particularly for neutron cross sections in the energy range from $1 \mathrm{keV}$ to $20 \mathrm{MeV}$.

In this review we assume that adequate cross sections are available from libraries, and therefore we do not present a comprehensive discussion of cross sections. Photon cross sections are sometimes more difficult to obtain than are neutron cross sections, but Refs. 168 to 176 may be helpful in this regard.

\section{APPLICATIONS OF MONTE CARLO IN OTHER AREAS}

The Monte Carlo method for solving problems other than neutron and photon transport is the primary motivation for Refs. 177 to 204 . The report by Everett ${ }^{177}$ on the mathematical framework to include relativistic effects in particle-transport calculations is mentioned specifically since it may be of particular interest. This list of references involving applications outside the realm of neutron and photon transport is by no means complete. Our intention is that it may serve as a useful starting point for a literature search on the use of Monte Carlo to solve special problems.

\section{G NERAL REFERENCES ON THE MONTE CARLO METHOD}

\section{Brioks}

1. Y. A. Shreider (Ed.), The Monte Carlo Method, Pergamon Press, Inc., New York, 1966.

2. E. D. Cashwell and C. J. Everett, A Practical Manual on the Monte Carlo Method for Random Walk Problems, Pergamon Press, Inc., New York, 1959.

3. J. Spanier and E. M. Gelbard, Monte Carlo Principles and Neutron Transport Problems, Addison-Wesley Publishing Company, Inc., Reading, Mass., 1969.

4. J. M. Hammersley and D. C. Handscomb, Monte Carlo Methods, John Wiley \& Sons, Inc., New York, 1964. 
5. E. Parzen, Modern Probability Theory and Its Applications, John Wiley \& Sons, Inc., New York, 1960.

6. M. Lòve, Probability Theory, Van Nostrand Reinhold Company, New York, 1950.

7. Birger Jansson, Random Number Generators, Victor Petterson's Bokindustri Aktiebolag, Stockholm, 1966.

\section{Random Numbers and Sampling from Density Functions}

8. T. E. Hull and A. R. Dobell, Random Number Generators, SIAM (Soc. Ind. Appl. Math.) Rev. 4: 230-254 (1962).

9. D. H. Lehmer, Mathematical Methods in Large-Scale Computing Units, in Proceedings of a Symposium on Large-Scale Digital Calculating Machinery, pp. 141-146 (1949); published as Ann. Comput. Lab., Harvard Univ., 26 (1951).

10. H. A. Feldman, Effects of Faulty Random Number Generators on Monte Carlo Calculations, USAEC Report OR NL-TM-1625, Oak Ridge National Laboratory, 1967.

11. H. A. Meyer, L. S. Gephart, and N. L. Tasmussen, On the Generation and Testing of Random Digits, Report WADC-TR-54-55, Wright Air Development Center, 1954.

12. C. Taussky and J. Todd, Generation and Testing of Pseudo-Random Numbers, in Symposium on Monte Carlo Methods, H. A. Meyer (Ed.), pp. 15-28, John Wiley \& Sons, Inc., New York, 1956 .

13. J. Certaine, On a Sequence of Pseudo-Random Numbers of Maximal Length, J. Ass. Comput. Mach. 5: 353-356 (1958).

14. C. J. Everett and E. D. Cashwell, A Monte Carlo Sampler, USAEC Report LA-5061-MS, Los Alamos Scientific Laboratory, 1972.

15. H. Kahn, Applications of Monte Carlo, USAEC Report AECU-3259, Rand Corporation, 1954.

16. E. D. Cashwell, C. J. Everett, and G. D. Turner, A Method of Sampling Certain Probability Densities Without Inversion of Their Distribution Functions, USAEC Report LA-5407-MS, Los Alamos Scientific Laboratory, 1973.

17. C. J. Everett, E. D. Cashwell, and G. D. Turner, A New Method of Sampling the Klein-Nishina Probability Distribution for All Incident Photon Energies Above $1 \mathrm{kcV}$, USAEC Report LA-4663, Los Alamos Scientific Laboratory, 1971.

18. C. J. Everett and P. R. Stein, On Random Sequences of Integers, Bull. Amer. Math. Soc., 76: 349-351 (1970).

19. C. J. Everett and E. D. Cashwell, Approximation for the Inverse of the Klein-Nishina Probability Distribution, USAEC Report LA-3839, Los Alamos Scientific Laboratory, 1970.

20. A. Foderaro, History of and Some Speculations Concerning the Use of Decidedly Deterministic Sampling Numbers To Increase the Efficiency of Monte Carlo Calculations, in A Review of the Monte Carlo Method for Radiation Transport Calculations (Collected Papers of a Seminar, October 5-7, 1970), USAEC Report ORNL-RSIC-29, pp. 15-18, Oak Ridge National Laboratory, 1971.

21. W. A. Woolson, Semi-Random Sampling Techniques for Monte Carlo Calculations, in A Review of the Monte Carlo Method for Radiation Transport Calculations (Collected Papers of a Seminar, October 5-7, 1970), USAEC Report ORNL-RSIC-29, pp. 19-26, Oak Ridge National Laboratory, 1971.

\section{Scoring and Statistical Errors}

22. G. L. Burrows and D. B. MacMillan, Confidence Limits for Monte Carlo Calculations, $\mathrm{Nucl}$. Sci. Eng., 22: 384-385 (1965). 
23. D. B. MacMillan, Comparison of Statistical Estimators for Neutron Monte Carlo Calculations, Nuc. Sci. Eng., 26: 366-372 (1966).

24. E. M. Gelbard, L. A. Ondis II, and J. Spanier, A New Class of Monte Carlo Estimators, SIAM (Soc. Ind. Appl. Math.), 14:697-701 (1966).

25. J. Spanier, Two Pairs of Families of Estimators for Transport Problems, SIAM /Soc. Ind. Appl. Math.), 14: 702-713 (1966).

26. N. M. Steen, A Simple Method To Improve the Efficiency of the $\Sigma_{\mathrm{a}} / \Sigma_{\mathrm{t}}$ Estimator in Certain Monte Carlo Programs, USAEC Report WAPD-TM-609, Bettis Atomic Power Laboratory, 1966.

27. F. H. Clark, Variance of Certain Flux Estimators Used in Monte Carlo Calculations, Nucl. Sci. Eng., 27: 235-239 (1967).

28. W. A. Coleman, Mathematical Verification of a Certain Monte Carlo Sampling Technique and Applications of the Technique to Radiation Transport Problems, Nucl. Sci. Eng., 32:76-81 (1968).

29. T. M. Jordan, Advanced Monte Carlo Concepts: Methods and Applications, Trans. Amer. Nucl. Soc., 12: 945 (1969).

30. M. H. Kalos, On the Estimation of Flux at a Point by Monte Carlo, Nucl. Sci. Eng., 16: 111-117 (1963).

31. H. A. Steinberg and M. H. Kalos, Bounded Estimators for Flux at a Point in Monte Carlo, Nucl. Sci. Eng., $44:$ 406-412 (1971).

32. E. S. Troubetzkoy and M. O. Cohen, Flux-at-a-Point Estimation for Cylindrically Symmetric Problems, Trans. Amer. Nucl. Soc., 10: 402403 (1967).

33. H. A. Steinberg, Correlated Sampling and Its Implementation in the SAMCEP Code, in Proceedings of a Seminar, A Review of Calculations of Radiation Transport in Air, Theory, Techniques, and Computer Codes, USAEC Report ORNL-RSIC-33, pp. 159-169, Oak Ridge National Laboratory, 1972.

34. N. J. Curlee, Jr., and L. A. Ondis II, The Use of Correlated Sampling in Monte Carlo Calculation of Changes in Thermal Absorption, Trans. Amer. Nucl. Soc., 7 : 288-289 (1964).

35. H. Takahashi, Monte Carlo Method for Geometrical Perturbation and Its Application to the Pulsed Fast Reactor, Nucl. Sci. Eng., 41 : 259-270 (1970).

36. H. Amster, Determining Collision Variances from Adjoints, Nucl. Sci. Eng., 43: 114-116 (1971).

37. C. R. Baldini, L. B. Ballico, and M. M. Spano, Multistage Self-Improving Monte Carlo Method, Nucl. Instrum. Methods, 72: 317-320 (1969).

\section{Importance Sampling and Biasing}

38. J. Spanier, A New Multi-Stage Procedure for Systematic Variance Reduction in Monte Carlo, in Proceedings of Conference on New Developments in Reactor Mathematics and Applications, Idaho Falls, Idaho, March 29-31, 1971, USAEC Report CONF-710302, Vol. 2, pp. 760-770, 1971.

39. R. R. Coveyou, V. R. Cain, and K. J. Yost, Adjoint and Importance in Monte Carlo Application, Nucl. Sci. Eng., 27: 219-234 (1967).

40. H. Borgwaldt, Comparison of Three Methods To Control the Leakage of Particles in a Monte Carlo Game, in Proceedings of Conference on New Developments in Reactor Mathematics and Applications, Idaho Falls, Idaho, March 29-31, 1971, USAEC Report CONF-710302, Vol. 2, pp. 857-870, 1971.

41. F. H. Clark, The Exponential Transform as an Importance-Sampling Device - A Review, USAEC Report ORNL-RSIC-14, Oak Ridge National Laboratory, 1966.

42. R. H. Karcher, R. C. Erdmann, and O. C. Baldonado, The Application of Track-Length Distribution Biasing in Monte Carlo Deep-Penetration Calculations, Nucl. Sci. Eng., 31: 492-499 (1968). 
43. V. R. Cain, E. A. Straker, and G. Thayer, Monte Carlo Path Length Selection Routines Based on Some Specific Forms of the Importance Function, USAEC Report ORNL-TM-1967, Oak Ridge National Laboratory, 1969.

44. J. M. Lanore, Weighting and Biasing of a Monte Carlo Calculation for Very Deep Penetration of Radiation, Nucl. Sci. Eng., 45: 66-72 (1971).

45. C. E. Burgart, and P. N. Stevens, General Method of Importance Sampling the Angle of Scattering in Monte Carlo Calculations, Nucl. Sci. Eng., 42: 306-323 ((1970).

46. A. B. Chilton, Linear Energy Transformation for Gamma-Ray Monte Carlo Calculations, Nucl. Sci. Eng., 34: 328-329 (1968).

47. C. Ponti, Angular and Track-Length Distribution Biasing in Monte Carlo Deep Penetration Calculations, in A Review of the Monte Carlo Method for Radiation Transport Calculations (Collected Papers of a Seminar, October 5-7, 1970), USAEC Report ORNL-RSIC-29, pp. 27-33, Oak Ridge National Laboratory, 1971.

48. F. A. Schmidt, E. A. Straker, and V. R. Cain, Application of Adjoint Flux Calculations to Monte Carlo Biasing, USAEC Report ORNL-TM-2454, Oak Ridge National Laboratory, 1968.

49. H. A. Steinberg, M. H. Kalos, and E. S. Troubetzkoy, Machine Generation of Monte Carlo Sampling Functions, in Proceedings of Conference on New Developments in Reactor Mathematics and Applications, Idaho Falls, Idaho, March 29-31, 1971, USAEC Report CONF-710302, Vol. 2, pp. 771-789, 1971.

50. H. A. Steinberg, M. H. Kalos, and E. Troubetzkoy, Automatic Computation of Importance Sampling Functions for Monte Carlo Transport Codes: Phase II, Report AD-716453, Mathematical Applications Group, Inc., 1970.

51. D. B. MacMillan, Optimization of Importance-Sampling Parameters in Monte Carlo, Nucl. Sci. Eng., 48: 219 (1972).

\section{Neutron Transport with Monte Carlo}

52. L. B. Miller and G. H. Miley, Some Monte Carlo Techniques for the Calculation of Doppler Coefficients, Nucl. Sci. Eng., 40: 438-448 (1970).

53. G. Goertzel and M. H. Kalos, Monte Carlo Methods in Transport Problems, in Progress in Nuclear Energy, Physics and Mathematics, Series I, Vol. 2, pp. 315-369, Pergamon Press, Inc., New York, 1958.

54. H. Rief and H. Kschwendt, Reactor Analysis by Monte Carlo, Nucl. Sci. Eng., 30: 395-418 (1967).

55. J. Spanier, Monte Carlo Methods and Their Application to Neutron Transport Problems, USAEC Report WAPD-195, Bettis Atomic Power Laboratory, 1959.

56. M. H. Kalos, F. R. Nakache, and J. Celnik, Monte Carlo Methods in Reactor Computations, in Computing Methods in Reactor Physics, pp. 359-438, H. Greenspan, C. N. Kelber, and D. Okrent (Eds.), Gordon and Breach, Science Publishers, Inc., New York, 1968.

57. L. L. Carter et al., Monte Carlo Code Development in Los Alamos, USAEC Report LA-5903-MS, Los Alamos Scientific Laboratory, March 1975.

58. F. G. Bischoff, M. L. Yeater, and W. E. Moore, Monte Carlo Evaluation of Multiple Scattering and Resolution Effects in Double-Differential Neutron Scattering Cross-Section Measurements, Nucl. Sci. Eng., 48: 266-280 (1972).

59. R. R. Coveyou, A Monte Carlo Technique for Selecting Neutron Scattering Angles from Anisotropic Distributions, Nucl. Sci. Eng., 21: 260-262 (1965).

60. L. L. Carter, E. D. Cashwell, and W. M. Taylor, Monte Carlo Sampling with Continuously Varying Cross Sections Along Flight Paths, Nucl. Sci. Eng., 48: 403-411 (1972).

61. C. J. Everett and E. D. Cashwell, Scattering Formulas for the Two-Particle Reaction, USAEC Report LA-5196-MS, Los Alamos Scientific Laboratory, 1973. 
62. J. Kimlinger and E. F. Plechaty, SORS Monte Carlo Neutron-Transport Code for the CDC-6600, USAEC Report UCRL-50532, University of California Lawrence Livermore Laboratory, 1968.

63. E. D. Cashwell, J. R. Neergaard, W. M. Taylor, and G. D. Turner, MCN: A Neutron Monte Carlo Code, USAEC Report LA-4751, Los Alamos Scientific Laboratory, 1972.

64. E. A. Straker, W. H. Scott, Jr., and N. R. Byrn, MORSE General Purpose Monte Carlo Multigroup Neutron and Gamma-Ray Transport Code with Combinatorial Geometry, RSIC Computer Code Collection Report CCC-127D, Oak Ridge National Laboratory, 1972.

65. E. A. Starker, Deciphering the Morse Code, in A Review of the Monte Carlo Method for Radiation Transport Calculations (Collected Papers of a Seminar, October 5-7, 1970), USAEC Report ORNL-RSIC-29, pp. 97-112, Oak Ridge National Laboratory, 1971.

66. W. H. Scott, Jr., and D. E. Groce, Experience with and Modifications to the Monte Carlo Code - Morse, in A Review of the Monte Carlo Method for Radiation Transport Calculations (Collected Papers of a Seminar, October 5-7, 1970), USAEC Report ORNL-RSIC-29, pp. 113-123, Oak Ridge National Laboratory, 1971.

67. M. L. Tobias, S. N. Cramer, R. S. Carlsmith, G. W. Perry, O. W. Hermann, J. L. Lucius, and G. W. Morrison, Application of the ESP Code to Two Neutron Distribution Problems, in a Review of the Monte Carlo Method for Radiation Transport Calculations (Collected Papers of a Seminar, October 5-7, 1970), USAEC Report ORNL-RSIC-29, pp. 75-83, Oak Ridge National Laboratory, 1971.

68. E. S. Troubetzkoy, UNC-SAM-2: A FORTRAN Monte Carlo Program Treating Time-Dependent Neutron and Photon Transport Through Matter, Report UNC-5157, United Nuclear Corporation, 1966.

69. T. M. Jordan, The Revised FASTER Program, in A Review of the Monte Carlo Method for Radiation Transport Calculations (Collected Papers of a Seminar, October 5-7, 1970), USAEC Report ORNL-RSIC-29, pp. 85-89, Oak Ridge National Laboratory, 1971.

70. W. E. Vesely, F. J. Wheeler, and R. S. Marsden, The Raffle Monte Carlo Code and Its Use To Obtain Correlated Estimates of Collapsed Cross Sections, in Proceedings of Conference on New Developments in Reactor Mathematics and Applications, Idaho Falls, Idaho, March 29-31, 1971, USAEC Report CONF-710302, Vol. 2, pp. 833-856, 1971.

71. D. R. Harris, D. R. Koenig, and W. Preeg, Multigroup Monte Carlo and $S_{\mathbf{n}}$ Methods for Air Transport, in Proceedings of a Seminar, A Review of Calculations of Radiation Transport in Air, Theory, Techniques, and Computer Codes, USAEC Report ORNL-RSIC-33, pp. 209-228, Oak Ridge National Laboratory, 1972.

72. W. Rothenstein, Monte Carlo Code for the Calculation of Resonance Reaction Rates and Effective Resonance Integrals Based on ENDF/B Data (REPCDC), USAEC Report BNL-13851, Brookhaven National Laboratory, 1969.

73. D. C. Irving, R. M. Freestone, Jr., and F. B. Kam, O5R General Purpose Monte Carlo Neutron Transport Code, USAEC Report ORNL-3622, Oak Ridge National Laboratory, 1965.

74. P. H. Keir and A. A. Robba, RABBLE, A Program for Computation of Resonance Absorption in Multi-Region Reactor Cells, USAEC Report ANL-7326, Argonne National Laboratory, 1967.

75. V. R. Cain, SAMBO, A Collision Analysis Package for Monte Carlo Codes, USAEC Report ORNL-TM-3203, Oak Ridge National Laboratory, 1970.

76. H. A. Steinberg, A Monte Carlo Air Transport Code with a Time Dependent Shock Wave, in Proceedings of a Seminar, A Review of Calculations of Radiation Transport in Air, Theory, Techniques, and Computer Codes, USAEC Report ORNL-RSIC-33, pp. 239-250, Oak Ridge National Laboratory, 1972.

77. MCNG: A Coupled Monte Carlo Neutron-Gamma Transport Code, Los Alamos Scientific Laboratory, in preparation.

78. P. A. Robinson, Jr., The Development and Application of a Coupled Monte Carlo Neutron-Photon Transport Code, USAEC Report UCRL-51234, University of California Lawrence Livermore Laboratory, 1972. 
79. N. R. Candelore and R. C. Gast, RECAP-2, A Monte Carlo Program for Estimating Epithermal Capture Rates in Rod Arrays, USAEC Report WAPD-TM-427, Bettis Atomic Power Laboratory, 1964.

80. E. A. Straker W. W. Engle, and P. N. Stevens, Some Calculated Milestone Solutions to Time-Dependent Radiation Transport Problems, USAEC Report ORNL-TM-2499, Oak Ridge National Laboratory, 1969.

81. J. J. Volpe, J. Hardy, Jr., and D. Klein, A Comparison of Thermal-Neutron Activation Measurements and Monte Carlo Calculations in Light-Water-Moderated Uranium Cells, Nucl. Sci. Eng., 40: 116-127 (1970).

82. J. J. Volpe and W. Baer, Monte Carlo Calculation of the Thermal Neutron Activation in Light-Water-Moderated Uranium Cells Using ENDF/B Cross-Section Data, Nucl. Sci. Eng., 43: 105-106 (1971).

83. R. H. Karcher, Neutron Air-Transport Data for the Design of Protective Structures, Nucl. Sci. Eng., 27: 367-387 (1967).

84. M. R. Mendelson, The Effect of Anisotropic Scattering in Plane-Geometry Transport Theory and Monte Carlo Calculations, Nucl. Sci. Eng., 28: 127-132 (1967).

85. P. F. Palmedo, Anisotropic Neutron Slowing Down in Aluminum-Water Mixtures. II: Monte Carlo Calculations, Nucl. Sci. Eng., 32: 313-318 (1968).

86. B. A. Zolotar, Monte Carlo Analysis of Nuclear Reactor Fluctuation Models, Nucl. Sci. Eng., 31: 282-294 (1968).

87. V. R. Cain, Comparisons of Monte Carlo Calculations to Measurements of Neutron Leakage from the TSF-SNAP Reactor, Nucl. Sci. Eng., 41: 310-315 (1970).

88. C. Delivers, Comparative Performances of Monte Carlo and Discrete Ordinate One Dimensional Transport Codes in an Iron Bulk Shield Calculation, in A Review of the Monte Carlo Method for Radiation Transport Calculations (Collected Papers of a Seminar, October 5-7, 1970), USAEC Report ORNL-RSIC-29, pp. 41-57, Oak Ridge National Laboratory, 1971.

89. D. G. Lindstrom, Discrete Ordinates-Monte Carlo Coupling, in A Review of the Monte Carlo Method for Radiation Transpcrt Calculations (Collected Papers of a Seminar, October 5-7, 1970), USAEC Report ORNL-RSIC-29, pp. 91-96, Oak Ridge National Laboratory, 1971.

90. N. E. Banks, Cross Section Sensitivity Studies Using the SAMCEP Code, in Proceedings of a Seminar, A Review of Calculations of Radiation Transport in Air, Theory, Techniques, and Computer Codes, USAEC Report ORNL-RSIC-33, pp. 171-196, Oak Ridge National Laboratory, 1972.

91. W. Matthes, Some Applications of the Monte Carlo Method at the Euratom Research Center Ispra, in Proceedings of Conference on New Developments in Reactor Mathematics and Applications, Idaho Falls, Idaho, March 29-31, 1971, USAEC Report CONF-710302, Vol. 2, pp. 803-832, 1971.

92. W. A. Woolson and W. A. Coleman, General Methods for Calculating the Effect of Source Anisotropy, in Proceedings of a Seminar, A Review of Calculations of Radiation Transport in Air, Theory, Techniques, and Computer Codes, USAEC Report ORNL-RSIC-33, pp. 361 400 , Oak Ridge National Laboratory, 1972.

93. H. Lichtenstein and L. J. Lidofsky, Monte Carlo Transport in Spatially Perturbed Media, in Proceedings of Conference on New Developments in Reactor Mathematics and Applications, Idaho Falls, Idaho, March 29-31, 1971, USAEC Report CONF-710302, Vol. 2, pp. 790-793, 1971.

94. R. J. Howerton, Predictions of the Energy Dependence of the Average Yield of Neutrons Per Fission of Isotopes of Thorium, Uranium, and Plutonium, Nucl. Sci. Eng., 46: 42-52 (1971).

95. J. J. Berlijn, R. E. Hunter, and C. C. Cremer, Neutron Cross Sections for ${ }^{235} \mathrm{U}$ and ${ }^{238} \mathrm{U}$ in the Energy Range $1 \mathrm{keV}$ to $14 \mathrm{MeV}$, USAEC Report LA-3527, Los Alamos Scientific Laboratory, 1968. 
96. R. E. Hunter, J. J. Berlijn, and C. C. Cremer, Neutron Cross Sections for ${ }^{239} \mathrm{Pu}$ and $240 \mathrm{Pu}$ in the Energy Range $1 \mathrm{keV}$ to $14 \mathrm{MeV}$, USAEC Report LA-3528, Los Alamos Scientific Laboratory, 1968.

97. C. W. Drawbaugh, On the Solution of Transport Problems by Conditional Monte Carlo, Nucl. Sci. Eng., 9: 185-197 (1961).

98. M. R. Mendelson, Applications of Monte Carlo Methods at Knolls Atomic Power Laboratory, Trans. Amer. Nucl. Soc., 11: 309 (1968).

99. B. R. Sehgal, Monte Carlo Calculations of Resonance Integral of 232 Th, Nucl. Sci. Eng., 27 : 95-103 (1967).

100. C. J. Everett, E. D. Cashwell, and R. G. Schrandt, Monte Carlo Transport Routine for the "U.S. Standard Atmosphere" (1962) to an Altitude of 90 Kilometers, USAEC Report LA-5089, Los Alamos Scientific Laboratory, 1972.

101. R. E. Maerker and F. J. Muckenthaler, A Benchmark Experiment for Neutron Transport in Iron and Stainless Steel, Nucl. Sci. Eng., 52: 227-246 (1973).

102. L. F. Hansen, J. D. Anderson, R. J. Doyas, R, J. Howerton, T. Komoto, C. M. Logan, and C. Wong, Measurements and Calculations of the Neutron Spectra Emitted from Concrete Spheres Bombarded with 14-MeV Neutrons, Nucl. Sci. Eng., 55: 345-348 (1974).

103. R. Kraft and C. J. Wensrich, Monte Carlo Methods. A Bibliography Covering the Period 1949 to 1963, USAEC Report UCRL-7823, University of California Lawrence Radiation Laboratory, 1964.

\section{Criticality}

104. G. E. Whitesides, Adjoint Biasing in Monte Carlo Criticality Calculations, Trans. Amer. Nucl. Soc., 11: 159 (1968).

105. J. B. Parker and E. R. Woodcock, Monte Carlo Criticality Calculations, in Progress in Nuclear Energy, Technology, Engineering, and Safety, Series IV, Vol. 4, pp. 435-457, Pergamon Press, Inc., New York, 1961.

105a. E. M. Gelbard (Chairman), Proceedings of the NEACRP Meeting of a Monte Carlo Study Group, USAEC Report ANL-75-2, Argonne National Laboratory, 1974.

106. D. H. Davis, Critical Size Calculations for Neutron Systems by the Monte Carlo Method, in Methods in Computational Physics, Vol. 1, pp. 67-68, Academic Press Inc., New York, 1963.

107. W. Matthes, Calculation of Reactivity Perturbations with the Monte Carlo Method, Nucl. Sci. Eng., 47: 234-237 (1972).

108. G. E. Whitesides, Monte Carlo Methods as Applied to Nuclear Criticality Safety Calculations, Trans. Amer. Nucl. Soc., 13: 392 (1970).

109. M. R. Mendelson, Monte Carlo Criticality Calculations for Thermal Reactors, Nucl. Sci. Eng., 32: 319-331 (1968).

110. J. Lieberoth, Monte Carlo Technique To Solve the Static Eigenvalue Problem of the Boltzmann Transport Equation, Nukleonik, 11:213-219 (1968).

111. L. L. Carter and N. J. McCormick, Source Convergence in Monte Carlo Calculations, Nucl. Sci. Eng., 36: 438-441 (1969).

112. D. B. MacMillan, Monte Carlo Confidence Limits for Iterated-Source Calculations, Nucl. Sci. Eng., 50: 73-74 (1973).

113. R. C. Gast and N. R. Candelore, Monte Carlo Eigenfunction Uncertainties, Trans. Amer. Nucl. Soc., 14: 219 (1971).

114. J. T. Mihalczo, Criticality of Graphite- and Polyethylene-Reflected Uranium(93.2\%)-Metal Cylinders and Annuli, Nucl. Sci. Eng., 49: $489-504$ (1972).

115. J. T. Mihalczo, Multiplication Factor of Uranium Metal by One-Velocity Monte Carlo Calculations, USAEC Report ORNL-P-2033, Oak Ridge National Laboratory, 1965. 
116. J. T. Thomas, Some Effects of Interspersed Moderation on Array Criticality, USAEC Report Y-CDC-6, Union Carbide Corporation, 1969.

117. G. E. Whitesides and J. T. Thomas, The Use of Differential Current Albedos in Monte Carlo Criticality Calculations, Trans. Amer. Nucl. Soc., 12: 889 (1969).

118. G. E. Whitesides, G. W. Morrison, and E. C. Crume, Few-Group Monte Carlo Criticality Calculations, Trans. Amer. Nucl. Soc., 9: 133-134 (1966).

119. G. E. Whitesides, A Difficulty in Computing the k-Effective of the World, Trans. Amer. Nucl. Soc., 14: 680 (1971).

120. G. E. Whitesides, The Monte Carlo Method as Applied to Nuclear Criticality Safety Calculations, USAEC Report Y-CDC-11, p. 19, Union Carbide Corporation, 1972.

121. J. T. Thomas, Critical Three-Dimensional Arrays of U(93.2)-Metal Cylinders, Nucl. Sci. Eng., 52: 350-359 (1973).

122. G. E. Whitesides and N. F. Cross, A Multigroup Monte Carlo Criticality Program, USAEC Report CTR-5, Union Carbide Computing Technology Center, 1969.

123. R. C. Lloyd, S. R. Bierman, and E. D. Clayton, Criticality of Plutonium-Uranium Mixtures Containing 5 to $8 \mathrm{Wt}$.\% Plutonium, Nucl. Sci. Eng., 55: $51-57$ (1974).

124. S. R. Bierman and E. D. Clayton, Critical Experiments To Measure the Neutron Poisoning Effects of Copper and Copper-Cadmium Plates, Nucl. Sci. Eng., 55: 58-66 (1974).

\section{Gamma-Ray Transport with Monte Carlo}

125. E. D. Cashwell, J. R. Necrgatard, C. J. Everctt, R. G. Schrandt, W. M. Taylor and G. D. Turner, Monte Carlo Photon Codes: MCG and MCP, USAEC Report LA-5157-MS, Los Alamos Scientific Laboratory, 1973.

126. E. A. Straker, The Effect of the Ground on the Steady-State and Time-Dependent Transport of Neutrons and Secondary Gamma Rays in the Atmosphere, Nucl. Sci. Eng., 46: 334-355 (1971).

127. J. W. Kimlinger, E. F. Plechaty, and J. R. Terrall, SORS Monte Carlo Photon-Transport Code, USAEC Report UCRL-50358, University of California Lawrence Radiation Laboratory, 1967.

128. W. A. Coleman, Monte Carlo Calculation of the Effect of Subterranean Perturbations on Reflected X-Rays, Nucl. Sci. Eng., 46:12-21 (1971).

129. A. Razan and H. E. Hungerford, A Three-Dimensional Stochastic Gamma-Ray Transport Method for Shielding Calculations, Nucl. Sci. Eng., 46: 1-11 (1971).

130. A. R. Buhl, Gamma Differential Energy Flux at Several Altitudes Above an Air-Ground Interface Fallout Field, Trans. Amer. Nucl. Soc., 12: 374-376 (1969).

131. F. Vesely, F. Bensch, and H. Hejtmanek, Monte Carlo Program for the Determination of the Energy Spectrum of Photoneutron Sources, Nucleonik, 11:300-303 (1968).

132. E. D. Cashwell, C. J. Everett, and W. M. Taylor, A General Monte Carlo Photon Code, in A Review of the Monte Carlo Method for Radiation Transport Calculations (Collected Papers of a Seminar, October 5-7, 1970), USAEC Report ORNL-RSIC-29, pp. 59-62, Oak Ridge National Laboratory, 1971.

133. L. L. Carter, E. D. Cashwell, and R. G. Schrandt, The Simulation of Low Energy Photon Transport and the Simulation of the Adjoint Neutron Transport Equation with Monte Carlo, in Proceedings of a Seminar, A Review of Calculations of Radiation Transport in Air, Theory, Techniques, and Computer Codes, USAEC Report ORNL-RSIC-33, pp. 197-208, Oak Ridge National Laboratory, 1972.

134. E. A. Straker, Comparisons of Calculations with Integral Experiments with Emphasis on Secondary Gamma Rays, in Proceedings of a Seminar, A Revicw of Calculations of Radiation Transport in Air, Theory, Techniques, and Computer Codes, USAEC Report ORNL-RSIC-33, pp. 117-155. Oak Ridge National Laboratory, 1972. 
135. P. A. Robinson, Jr., and G. D. Stouter, Inverse Compton Scattering as an Energy Loss Mechanism in a Controlled Thermonuclear Reactor, Nucl. Sci. Eng., 49: 117-129 (1972).

136. C. D. Zerby, A Monte Carlo Calculation of the Response of Gamma-Ray Scintillation Counters, in Methods in Computational Physics, Vol. 1, pp. 89-134, Academic Press Inc., New York, 1963.

\section{Shielding}

137. P. S. Mittelman and M. H. Kalos, Practical Aspects of Multi-Dimensional Monte Carlo Shielding Calculations, Nucl. Eng. Des., 13: 409-414 (1970).

138. R. Nicks and H. Penkuhn, Today's Approaches to Reactor Shielding Problems, Nucl. Eng. Des., 15: 3, 294-308 (1971).

139. A. B. Chilton, A New Variant to the Exponential Transformation Technique in Monte Carlo Shiclding Calculations, Nucl. Sci. Eng., 24: 200-208 (1966).

140. T. M. Jordan, Advanced Monte Carlo Concepts in Radiation Shielding Calculations: Methods and Applications, Nucl. Eng. Des., 13: 415-422 (1970).

141. M. Z. Brainina, V. L. Generozov, V. G. Kuznetsov, and V. A. Sakovich, Calculation of Dose Derivatives by Monte Carlo for Optimization of Shape and Composition of Shields, USAEC Report ORNL-tr-1884, Oak Ridge National Laboratory, 1967.

\section{Monte Carlo Simulation of the Adjoint Equation}

142. L. L. Carter, MCNA: A Computer Program To Solve the Adjoint Neutron Transport Equation by Coupled Sampling with the Monte Carlo Method, USAEC Report LA-4488, Los Alamos Scientific Laboratory, 1971.

143. L. B. Levitt and J. Spanier, A New Non-Multigroup Adjoint Monte Carlo Technique, Nucl. Sci. Eng., 37: 278-287 (1969).

144. D. C. Irving, The Adjoint Boltzmann Equation and Its Simulation by Monte Carlo, USAEC Report ORNL-TM-2870, Oak Ridge National Laboratory, 1970.

145. B. Eriksson, C. Johansson, M. Leimdorfer, and M. H. Kalos, Monte Carlo Integration of the Adjoint Neutron Transport Equation, Nucl. Sci. Eng., 37: 410-422 (1969).

146. L. L. Carter and N. J. McCormick, Coupled Sampling with the Monte Carlo Method in Neutron Transport Calculations, Nucl. Sci. Eng., 39: 296-310 (1970).

147. C. W. Maynard, An Application of the Reciprocity Theorem to the Acceleration of Monte Carlo Calculations, Nucl. Sci. Eng., 10: 97-101 (1961).

148. M. H. Kalos, Monte Carlo Integration of the Adjoint Gamma-Ray Transport Equation, $\mathrm{NuCl}$. Sci. Eng., 33: 284-290 (1968).

149. G. W. Morrison, G. E. Whitesides, and D. C. Irving, Adjoint Monte Carlo Calculations for Enriched-Uranium-Metal Assemblies, Trans. Amer. Nucl. Soc., 9: 440-441 (1966).

150. M. O. Cohen, Neutron Adjoint Monte Carlo Calculations, Trans. Amer. Nucl. Soc., 12: 403 (1969).

151. M. H. Kalos, Adjoint Monte Carlo - What Is It? Who Needs It?, in A Review of the Monte Carlo Method for Radiation Transport Calculations (Collected Papers of a Seminar, October 5-7, 1970), USAEC Report ORNL-RSIC-29, pp. 1-6, Oak Ridge National Laboratory, 1971.

152. L. L. Carter, The Utilization of Coupled Sampling To Solve the Adjoint Neutron Transport Equation, in A Review of the Monte Carlo Method for Radiation Transport Calculations (Collected Papers of a Seminar, October 5-7, 1970), USAEC Report ORNL-RSIC-29, pp. 7-10, Oak Ridge National Laboratory, 1971.

153. M. O. Cohen, ANTE 2 and Its Uses in a Representative Calculation, in A Review of the 
Monte Carlo Method for Radiation Transport Calculations (Collected Papers of a Seminar, October 5-7, 1970), USAEC Report ORNL-RSIC-29, pp. 145-151, Oak Ridge National Laboratory, 1971.

154. J. Spanier and G. J. Habetler, Application of Monte Carlo Methods to Neutron Thermalization, in Naval Reactor Physics Handbook, Vol. I, pp. 379-532, A. Radkowsky (Ed.), USAEC Report TID-7030, Superintendent of Documents, U.S. Government Printing Office, Washington, 1974.

\section{Nonlinear Radiative Transport}

155. J. A. Fleck, Jr., and J. D. Cummings, An Implicit Monte Carlo Scheme for Calculating Time and Frequency Dependent Nonlinear Radiation Transport, J. Comput. Phys., 8: 313-342 (1971).

156. L. L. Carter and C. A. Forest, Nonlinear Radiation Transport Simulation with an Implicit Monte Carlo Method, USAEC Report LA-5038, Los Alamos Scientific Laboratory, 1973.

157. J. A. Fleck, Jr., The Calculation of Nonlinear Radiation Transport by a Monte Carlo Method, in Methods in Computational Physics, Vol. 1, pp. 43-65, Academic Press, Inc., New York, 1963.

158. G. S. Fraley, The Integrated Compton Cross Section and Its Use in a Monte Carlo Scheme, USAEC Report LA-4592, Los Alamos Scientific Laboratory, 1971.

159. J. Brooks, M. H. Kalos, H. A. Steinberg, and E. S. Troubetzkoy, Non-Linear Monte Carlo, in Proceedings of a Conference on New Developments in Reactor Mathematics and Applications, Idaho Falls, Idaho, March 29-31, 1971, USAEC Report CONF-710302, Vol. 2, pp. 794-797, 1971.

\section{Cross Sections}

160. E. D. Cashwell and E. F. Plechaty, Specification and Testing of Nuclear Data Required for Monte Carlo Transport Calculations, Nucl. Sci. Eng., 49: 394 (1972).

161. L. B. Levitt, The Probability Table Method for Treating Unresolved Neutron Resonances in Monte Carlo Calculations, Nucl. Sci. Eng., 49: $450-457$ (1972).

162. D. E. Cullen, Application of the Probability Table Method to Multigroup Calculations of Neutron Transport, Nucl. Sci. Eng., 55: 387-400 (1974).

163. M. K. Drake (Ed.), Data Formats and Procedures for the ENDF Neutron Cross Section Library, USAEC Report BNL-50274, ENDF-102, Vol 1, Brookhaven National Laboratory, October 1970.

164. D. J. Dudziak (Comp. and Ed.), ENDF Formats and Procedures for Photon Production and Interaction Data, USAEC Report LA-4549, ENDF-102 (Rev.), Vol. 2, Los Alamos Scientific Laboratory, July 1971.

165. M. A. Abdou and R. W. Conn, A Comparative Study of Several Fusion Reactor Blanket Designs, Nucl. Sci. Eng., 55: 256-266 (1974).

166. M. A. Abdou, C. M. Maynard, and C. Q. Wright, MACK: A Computer Program To Calculate Neutron Energy Release Parameters (Fluence-to-KERMA Factors) and Multigroup Neutron Reaction Cross Sections from Nuclear Data in ENDF Format, USAEC Report ORNL-TM-3994, Oak Ridge National Laboratory, 1973.

167. R. J. Howerton, R. J. Doyas, T. C. Michels, and S. T. Perkins, An Integrated System for Production of Neutronics and Photonics Calculational Constants, Vol. 4, Evaluated Nuclear Cross Section Library, USAEC Report UCRL-50400, University of California Lawrence Radiation Laboratory, 1971. 
168. Graphs of the Compton Energy-Angle Relationship and the Klein-Nishina Formula from 10 $\mathrm{keV}$ to $500 \mathrm{MeV}$, National Bureau of Standards, Circular No. 542, Superintendent of Documents, U.S. Government Printing Office, Washington, 1953.

169. D. T. Cromer and J. B. Mann, Compton Scattering Factors for Spherically Symmetric Free Atoms, J. Chem. Phys., 47: 1892-1893 (1967).

170. D. T. Cromer, Compton Scattering Factors for Aspherical Free Atoms, J. Chem. Phys., 50: $4857-4859$ (1969).

171. R. T. Brown, Edgerton, Germeshausen \& Grier, Inc., Technical Report L-958, 1969, and unpublished data.

172. G. W. Grodstein, X-Ray Attenuation Coefficients from $10 \mathrm{keV}$ to $100 \mathrm{MeV}$, National Bureau of Standards, Circular No. 583, Superintendent of Documents, U. S. Government Printing Office, Washington, 1957.

173. P. A. Doyle and P. S. Turner, Relativistic Hartree-Fock X-Ray Electron Scattering Factors, Acta Cryst., 24: 390-397 (1968).

174. D. T. Cromer and J. T. Waber, Atomic Scattering Factors for X-Rays, in International Tables for X-Ray Crystallography, Vol. 4, pp. 71-147, Kynoch Press, Birmingham, England, 1974.

175. H. P. Hanson, F. Herman, J. D. Lea, and S. Skillman, HFS A tomic Scattering Factors, Acta Cryst., 17: 1040-1044 (1964).

176. E. Storm and H. I. Israel, Photon Cross Sections from $1 \mathrm{keV}$ to $100 \mathrm{MeV}$ for Elements $\mathrm{Z}=1$ to $\mathrm{Z}=100$, At. Data Nucl. Data Tables, 7: 565-681 (1970).

\section{Monte Carlo References for Other Than Neutron and Gamma-Ray Transport}

177. C. J. Everett, A Relativity Notebook for Monte Carlo Practice, USAEC Report LA-3839, Los Alamos Scientific Laboratory, 1967.

178. C. D. Zerby and F. L. Keller, Electron Transport Theory, Calculations, and Experiments, Nucl. Sci. Eng., 27: 190-218 (1967).

179. T. W. Armstrong and R. G. Alsmiller, Jr., Monte Carlo Calculations of the Nucleon-Meson Cascade in Iron Initiated by $1-$ and 3- GeV Protons and Comparison with Experiment, $\mathrm{Nucl}$. Sci. Eng., 33: 291-296 (1968).

180. T. W. Armstrong, Monte Carlo Calculations of Residual Nuclei Production in Thick Iron Targets Bombarded by 1 and $3 \mathrm{GeV}$ Protons and Comparisons with Experiment, J. Geophys. Res., 74: 1361-1373 (1969).

181. J. J. Thompson, and P. Y. Chein, Heat Conduction with Internal Sources by Modified Monte Carlo Methods, Nucl. Eng. Des., 12: 207-214 (1970).

182. M. H. Kalos, Monte Carlo Calculations of the Ground State of Three- and Four-Body Nuclei, Phys. Rev., 128: 1791-1795 (1962).

183. K. Chen, G. Friedlander, and J. M. Miller, Effects of Using a Velocity-Dependent Potential in a Monte Carlo Simulation of Intranuclear Cascades, Phys. Rev., 176: 1208-1210 (1968).

184. L. W. Ehrlich, Monte Carlo Solutions of Boundary Value Problems Involving the Difference Alanog of $\left(\partial^{2} \mathrm{u} / \partial \mathrm{x}^{2}\right)+\left(\partial^{2} \mathrm{u} / \partial \mathrm{y}^{2}\right)+(\mathrm{K} / \mathrm{y})(\partial \mathrm{u} / \partial \mathrm{y})=0$, Ass. Comput. Mach. J., 6:204-218 (1959).

185. R. C. Herndon and Y. C. Tang, Variational Calculations in Few-Body Problems with Monte Carlo Method, in Methods in Computational Physics, Vol. 6, pp. 153-233, B. Alder, S. Fernback, and M. Rotenberg (Eds.), Academic Press, Inc., New York, 1966.

186. G. C. Geisler and R. E. Zindler, A Critical Time Step Approach to Monte Carlo Reliability Calculations, Nucl. Sci. Eng., 48: 255-265 (1972).

187. T. W. Armstrong, R. G. Alsmiller, Jr., K. C. Chandler, and B. L. Bishop, Monte Carlo Calculations of High-Energy Nucleon-Meson Cascades and Comparison with Experiment, Nucl. Sci. Eng., 49: 82-92 (1972). 
188. V. S. Barashenkov, N. M. Sobolevskii, and V. D. Toneev, Interactions of High-Energy Radiation with Matter (in Russian), At. Energ. (USSR), 32: 2, 123-129 (1972).

189. M. J. Berger, Monte Carlo Calculation of the Penetration and Diffusion of Fast Charged Particles, in Methods in Computational Physics, Vol. 1, pp. 135-215, Academic Press, Inc., New York, 1963.

190. R. T. Giuli and T. A. Moss, The Modulation of Galactic Protons by the Solar Wind-A Monte Carlo Approach, Astrophys. J., 167: 331-340 (1971).

191. L. L. House and L. C. Cohen, The Treatment of Resonance Scattering of Polarized Radiation in Weak Magnetic Fields by the Monte Carlo Technique, Astrophys. J., 157: 261-271 (1969).

192. H. Burfeindt, Monte Carlo Calculations for $3-\mathrm{GeV}$ Showers in Lead, USAEC Report ORNL-tr-1908, Oak Ridge National Laboratory, 1968.

193. S. Wittig, A Universal Monte Carlo Method for the Solution of Electron Transport Problems, USAEC Report ORNL-tr-2126, Oak Ridge National Laboratory, 1968.

194. R. E. Texter and V. V. Verbinski, 05S: A Monte Carlo Code for Calculating Pulse Height Distributions Due To Monoenergetic Neutrons Incident on Organic Scintillators, USAEC Report ORNL-4160, Oak Ridge National Laboratory, 1968.

195. T. Holtz and E. P. Muntz, Comparisons of Measured and Predicted Velocity Distribution Functions in a Shock Wave, Phys. Fluids, 14: 545-548 (1971).

196. J. F. Hague, Variance Reduction in the Monte Carlo Method for Determining the Volume of Multidimensional Nonanalytical Solids, Nucl. Instrum. Methods, 47: 194-200 (1967).

197. R. B. Carr, A Monte Carlo Solution to the Problem of Torsion of Prismatic Bars, USAEC Report UCRL-70600, University of California Lawrence Radiation Laboratory, 1967.

198. V. S. Barashenkov and K. K. Gudima, Computing Process for Intra-Nuclear Cascades, USAEC Report ORNL-tr-2130, Oak Ridge National Laboratory, 1968.

199. M. Treiman, Monte Carlo Methods for the Solution of Heat Transfer Problems, Including Hybrid Computer Techniques for Monte Carlo, USAEC Report LA-3777, Los Alamos Scientific Laboratory, 1967.

200. F. T. Wall, S. Windwer, and P. J. Gans, Monte Carlo Methods Applied to Configurations of Flexible Polymer Molecules, in Methods in Computational Physics, Vol. 1, pp. 217-243, Academic Press, Inc., New York, 1963.

201. L. D. Fosdick, Monte Carlo Computations on the ISING Lattice, in Methods in Computational Physics, Vol. 1, pp. 245-280, Academic Press, Inc., New York, 1963.

202. J. M. Hammersley, A Monte Carlo Solution of Percolation in the Cubic Crystal, in Methods in Computational Physics, Vol. 1, pp. 281-298, Academic Press, Inc., New York, 1963.

203. V. Staggs, The Information Handling Properties of Monte Carlo Programs: How To Solve Large Problems, in a Review of the Monte Carlo Method for Radiation Transport Calculations (Collected Papers of a Seminar, October 5-7, 1970), USAEC Report ORNL-RSIC-29, pp. 36-39, Oak Ridge National Laboratory, 1971.

204. B. S. Wang and G. H. Miley, Monte Carlo Simulation of Radiation-Induced Plasmas, Nucl. Sci. Eng., 52: 130-141 (1973). 
$\bullet$ 


\section{INDEX}

\section{Adjoint}

boundary conditions, 59

equation, $16,37,57$

reciprocity relation, 57

simulation, 55-60

use in biasing, 16, 37-38

Amster, H., 96

Antithetic variates, $19-25$

\section{Berger, M. J., 91}

Bernoulli trial, 26

Beyer, W. A., 3

Biasing

of adjoint, 60

antithetic variates, 19-25

Russian roulette, 16-17

source, 15, 36-37, 45-46

splitting, 16-17

stratified sampling, 25

using adjoint, 15-16, 36-38

Binomial distribution, 26

Blackbody radiation, 12

Boltzmann transport equation, 56

adjoint equation, 57

simulation of, $32-41$

Boundary conditions, 59

Brown, R. T., 83

Burrows, G. L., 96
Cain, V. R., 33, 39

Carter, L. L., 60

Cartesian coordinates, 43

Cashwell, E. D., 12, 22, 41, 46, 80, 95, 99

Central limit theorem, 28-29

Chebyshev's inequality, 26

Coherent scattering, 83-85

\section{Collision}

kernel, 48-49

reducing weight for capture, 49

sampling distance to, 5-6, 46

sampling event, 48-50

sampling isotope of, 49

Combined neutron-photon transport, 91,99

Compton scattering, 79-81

Correlated sampling, 25, 96

Correlation coefficient, 19

Covariance, 19

Coveyou, R. R., 3, 33, 39

Criticality, 75,77

Cromer, D. T., 83

Cullen, D. E., 100

Direction of flight

Cartesian coordinates, 43

after collision, 44

Dobell, A. R., 3, 95 
Edge energies, 86

Elastic scattering, 65-67

Eriksson, B., 60

Error evaluation and reduction

of adjoint simulation, 60

central limit theorem, 28-29

correlated sampling, 25,96

exponential transformation, $17-19,40$

Russian roulette, 16-17

splitting, 16-17

zero-variance estimator, $36-38$

Estimators

collision, 36, 50-51

last event, 36, 50-51

path length, $50-51$

point detector, $51-55,68-69,75-77$

zero variance, $36-38$

Everett, C. J., 12, 22, 41, 46, 80, 95, 99100

Expected value, 14, 28, 35, 47-48

Exponential transformation, 17-19, 40

Fission

in criticality calculations, 75,77

inclusion of inelastic neutrons, 70-71

sampling of energy spectrum, 12

sampling the event, 70-71

Fluorescence, 85-90

yield, 87

Foderaro, A., 95

Free gas kernel, 72-76

Gelbard, E. M., 2, 19, 22, 24-25, 33, 39, $55,63,95-96$

Geometrical considerations advancing particle, 43

Cartesian coordinates, 43 direction after scattering, 44 direction of flight, $43-44$

region boundaries, $44-45$

Goertzel, G., 16, 24-25, 33

Green's function, 56-57

Habetler, G. J., 60

Hammersley, J. M., 19, 24, 95

Handscomb, D. C., 19, 24, 95

Howerton, R. J., 100

Hull, T. E., 3, 95

Importance sampling, 13-16, 36-39

Incoherent scattering, 81-83
Inelastic scattering, 67-70

$\left(n, n^{\prime}\right)$ reaction, $67-70$

$(n, 2 n)$ and $(n, 3 n)$ inelastic events, 70

point-detector score for $\left(n, n^{\prime}\right)$

scattering, 68-69

statistical gas model, 69

Integral transport equation, $15,33,51$

adjoint, 16, 37

kernel, 33, 48-50

multicollision expansion of, 34

Integro-differential transport equation, $40-41,56$

Irving, D. C., 39-40

Isotropic distribution, 6

Israel, H. I., 84, 86-87, 90

Jansson, B., 3, 95

Kahn, H., 12, 81

Kalos, M. H., 16, 24-25, 33, 54

Kernel

normalized, 34

unnormalized, 34

Klein-Nishina cross section, 80

Knuth, D. E., 3

Kraft, R., 98

Last-event estimator, $36,50-51$

Law of large numbers, 26-27

Lehmer, D. H., 3,95

Leimdorfer, M., 19

Levitt, L. B., 19, 60, 100

Loeve, M., 95

McCormick, N. J., 60

MacLaren, M. D., 3

MacMillan, D. B., 55, 96

Mann, J. B., 83

Marotta, C. R., 86

Marsaglia, G., 3

Mean value, $14,26,28-29$

Mihalczo, J. T., 99

Miley, G. H., 96

Miller, L. B., 96

Monte Carlo

basic principle of, 2-7

codes

combined neutron-gamma, MCNG, 91

neutron

MARC, 63

MCN, 91 
MORSE, 63

TART, 63

photon

MCG, 91

MCP, 83-84, 86

PHOTRAN, 86

SORS, 84

multigroup, 61-63

results, accuracy of, 26-29

Morton, K. W., 19, 24

Neutron-photon transport (combined), 91, 99

Ondis, L. A., II, 55

Pair production, 90-91

Parzen, E., 95

Photoelectric effect, $85-90$

Point-detector estimator, 51-55, 68-69, 75-77

Probability density function, 3 distribution function, 3

Pseudorandom numbers, 3

Random numbers

generation of, 2-3

sampling with, 2-13

Reciprocity theorem, 57

Rejection technique, 8-11

efficiency of, 9

Russian roulette, 16-17

\section{Sampling}

adjoint equation, 55-60

altered density functions, $15-16,36-38$

common density functions, 12-13

correlated, 25, 96

with equally likely.intervals, 65-66

exponential distribution, 5-6, 46

isotropic direction, 6-7

rejection, 8-11

Russian roulette, 16-17

source, $45-46,75,77$

splitting, 16-17

stratified, 25

sum of positive functions, 13

type of collision, $3,48-50$

Scattering

coherent, 83-85
Compton, 79-81

elastic, $65-67$

factors, 81-85

free gas kernel, 72-76

incoherent, 81-83

inelastic, 67-70

Klein-Nishina, 80

statistical gas model, 69

thermal, 71-76

Thomson, 83

Scattering angle

center of mass, 65-67

laboratory, 66-67

new direction after scattering, 44

sampling from tables, 66

Seltzer, S. M., 91

Shreider, Y. A., 94

Source, $45-46$

biasing, $15,36-37,45-46$

iteration, 75,77

Spanier, J., 2, 19, 22, 24-25, 33, 39, 55, $60,63,95-96$

Splitting, 16-17

Statistical gas model, 69

Steinberg, H. A., 54

Storm, E., 84, 86-87, 90

Stratified sampling, 25

Surface description, 44-45

Takahashi, H., 96

Tee, G. J., 94

Thermal scattering, 71-76

point-detector score, $75-77$

Thompson, W. L., 91

Time dependence, 45

Transport equation integral form, 15, 33, 51

integro-differential form, 40-41, 56

Unbiased estimates, 35

Variance, 14-15, 19, 21-24, 27-29

Wensrich, C. J., 98

Woolson, W. A.. 95

Yost, K. J., 33, 39

Zero-variance estimator, $36-38$ 


\section{NOTICE}

This book was prepared as an account of work sponsored by the United States Government. Neither the United States nor the United States Energy Research and Development Administration, nor any of their employees, nor any of their contractors, subcontractors, or their employees, makes any warranty, express or implied, or assumes any legal liability or responsibility for the accuracy. completeness or usefulness of any information, apparatus, product or process disclosed, or represents that its use would not infringe privately owned rights. 


\title{
Reactor Shielding for Nuclear Engineers
}

\author{
By N. M. Schaeffer
}

Radiation Research Associates, Inc.

Prepared for the Division of Reactor Development and Technology, USAEC, and published by the Office of Information Services, USAEC

As the number of nuclear power plants on order continues to grow (currently more than thirty per year in the United States alone), the demand for nuclear engineers should also increase, and a new text on reactor shielding is overdue. Shielding technology has matured considerably in the last decade, and shield physics must routinely be translated into shield design. Since the publication in 1959 of Fundamental Aspects of Reactor Shielding, by Herbert Goldstein, new generations of computers have become available to exploit techniques heretofore considered too costly, and new measurement techniques have been devised. The energy and angular distributions of neutrons and gamma rays can be followed, both in theory and in practice, throughout their transport histories: Such powerful tools have brought correspondingly large dividends to the shielding community.

These advances and their underlying fundamentals are recorded in this volume, which is intended as a text for a two-semester course in reactor shielding directed at an advanced-undergraduate or a graduate level. ( 800 pages; 7 by 10 inches; Library of Congress Catalog Card Number 73-600001)

Available as TID-25951 for $\$ 13.60$ from National Technical Information Service U. S. Department of Commerce Springfield, Virginia 22161 


\title{
NUCLEAR POWER PLANT DESIGN ANALYSIS
}

\author{
Alexander Sesonske \\ Purdue University \\ Published by \\ Technical Information Center. \\ Office of Information Services, \\ U. S. Atomic Energy Commission \\ November 1973 \\ 495 pages, 151 illustrations, 6 by 9 inches \\ Library of Congress Catalog Card \\ Number 73-600245 \\ Available as TID-26241 for $\$ 10.60$ from \\ National Technical Information Service \\ U. S. Department of Commerce \\ Springfield, Virginia 22161

\section{CONTENTS} \\ Introduction \\ Electric Utility Economics \\ Nuclear-Plant Economics \\ Thermal-Transport Systems and Core Design \\ Nuclear Analysis Methods \\ Safety and Related Design Requirements \\ Fuel-System Analysis \\ Design Considerations \\ Some Optimization Approaches \\ Index
}

Nuclear Power Plant Design Analysis provides background in two basic areas: (1) design of nuclear power plants with emphasis on the reactor system and (2) design analysis, the systematic consideration of important individual parameters that affect the design in an interrelated way. It is a text for design-oriented courses for nuclear engineering students who have already had an introduction to the field but require additional perspective of interacting engineering areas important to reactor design. Emphasis is given to such areas as engineering economics, thermal-hydraulics, safety analysis, and fuel systems, which in an introductory course are often not covered with sufficient breadth to enable a student to participate in a meaningful group-design effort. The book is also intended for the practicing engineer interested in improving his background in reactor design.

Areas covered are principles of engineering design as they apply to nuclear projects; economics affecting nuclear power plants; thermal transport (thermal-hydraulics) in developing core specifications; computer methods for nuclear analysis; safety analysis, licensing, and environmental considerations; the fuel system and fuel-management strategies; the synthesis of all these design considerations; and modern optimization approaches and their potential usefulness in nuclear systems.

The author has taken full advantage of the report literature and other sources to obtain a unified treatment of topics not previously covered in a single book. An extensive bibliography is given to aid in additional study. 


\section{ERDA \\ CRITICAL \\ REVIEW \\ SERIES}

As a continuing series of state-of-the-art studies published by the ERDA Office of Public Affairs, the ERDA Critical Reviews are designed to evaluate the existing state of knowledge in a specific and limited field of interest, to identify significant developments, both published and unpublished, and to synthesize new concepts out of the contributions of many.

\section{SOURCES OF TRITIUM AND ITS BEHAVIOR UPON RELEASE \\ TO THE ENVIRONMENT \\ December 1968 (TID-24635) \$6.00 \\ D. G. Jacobs \\ Oak Ridge National Laboratory}

\section{REACTOR-NOISE ANALYSIS}

IN THE TIME DOMAIN

April 1969 (TID-24512) $\$ 6.00$

Nicola Pacilio

Argonne National Laboratory

and Comitato Nazionale

per l'Energia Nucleare

PLUME RISE

November 1969 (TID-25075) \$6.00

G. A. Briggs

Environmental Science Services

Administration

\section{ATMOSPHERIC}

TRANSPORT PROCESSES

EImar R. Reiter

Colorado State University

Part 1: Energy Transfers

and Transformations

December 1969 (TID-24868) $\$ 6.00$

Part 2: Chemical Tracers

January 1971 (TID-25314) $\$ 6.00$

Part 3: Hydrodynamic Tracers.

May 1972 (TID-25731) \$3.00

THE ANALYSIS OF

ELEMENTAL BORON

November 1970 (TID-25190) $\$ 3.00$

Morris W. Lerner

New Brunswick Laboratory

\author{
AERODYNAMIC CHARACTERISTICS \\ OF ATMOSPHERIC BOUNDARY LAYERS \\ May 1971 (TID-25465) $\$ 3.00$ \\ Erich J. Plate \\ Argonne National Laboratory \\ and Karlsruhe University
}

\section{NUCLEAR-EXPLOSION \\ SEISMOLOGY}

September 197i (TID-25572) $\$ 3.00$

Howard C. Rodean

Lawrence Livermore Laboratory

\section{BOILING CRISIS AND CRITICAL}

HEAT FLUX
August 1972 (TID-25887) $\$ 3.00$
L. S. Tong
Westinghouse Electric Corporation

\section{NEPTUNIUM-237}

PRODUCTION AND RECOVERY

October 1972 (TID-25955) $\$ 3.00$

Wallace W. Schulz and Glen E. Benedict

Atlantic Richfield Hanford Company

THE KINETICS OF THE OXIDATIONREDUCTION REACTIONS OF URANIUM, NEPTUNIUM, PLUTONIUM, AND AMERICIUM IN AQUEOUS SOLUTIONS

August 1975 (TID-26506) $\$ 5.45$

T. W. Newton

Los Alamos Scientific Laboratory,

University of California

\section{PARTICLE-TRANSPORT SIMULATION WITH THE MONTE CARLO METHOD October 1975 (TID 26607) $\$ 5.45$ \\ L. L. Carter and E. D. Cashwell \\ Los Alamos Scientific Laboratory}

Available from the National Technical Information Service, U. S. Department of Commerce, Springfield, Virginia 22161 